\title{
Dawn of a New Era in Sponge Biotechnology
}

Kylie van Deinsen-Hesp 


\section{Propositions}

1. Sponge cells in culture grow more like uni- than multicellular organisms (this thesis)

2. Sponges are both elegantly simple and intriguingly complex (this thesis)

3. Nuclear energy is the most realistic short-term option to stop climate change

4. The observed increase in mental health problems in slaughterhouse workers shows their work does not agree with human nature

5. Your negative experiences may not be your responsibility, but how you choose to deal with them is

6. Metal music enhances focus in a way comparable to noise-cancelling headphones

Propositions belonging to the thesis, entitled:

Dawn of a New Era in Sponge Biotechnology

Kylie van Deinsen-Hesp

Wageningen, February 16, 2021 



\section{Dawn of a New Era in Sponge Biotechnology}

Kylie van Deinsen-Hesp 


\section{Thesis committee}

\section{Promotors}

Prof. Dr. Shirley A. Pomponi

Research Professor

Harbor Branch Oceanographic Institute, Florida Atlantic University, USA

Emeritus Special Professor of Marine Biotechnology,

Wageningen University \& Research

Prof. Dr. Rene H. Wijffels

Professor of Bioprocess Engineering Group

Wageningen University \& Research

\section{Co-promotor}

Dr. Dirk E. Martens

Associate professor, Bioprocess Engineering Group

Wageningen University \& Research

\section{Other members}

Dr. Klaske J. Schippers, Max Planck Institute for Biophysical Chemistry, Göttingen, Germany Dr. Ronald Osinga, Marine Animal Ecology, Wageningen University \& Research

Prof. Dr. Huub F.J. Savelkoul, Cell biology and Immunology, Wageningen University \& Research Prof. Dr. Nicole J. de Voogd, Naturalis, Leiden, the Netherlands

This research was conducted under the auspices of the Graduate School VLAG (Advances studies in Food Technology, Agrobiotechnology, Nutrition and Health Science.) 


\title{
Dawn of a New Era in Sponge Biotechnology
}

\author{
Kylie van Deinsen-Hesp
}

submitted in fulfillment of the requirements for the degree of doctor at Wageningen University

by the authority of the Rector Magnificus,

Prof. Dr. A.P.J. Mol,

in the presence of the

Thesis Committee appointed by the Academic Board

to be defended in public

on Tuesday February 16, 2021

at 4 p.m. in the Aula. 
Kylie van Deinsen-Hesp

Dawn of a New Era in Sponge Biotechnology

PhD thesis, Wageningen University, Wageningen, the Netherlands (2020) With references, with summary in English

ISBN: 978-94-6395-693-2

DOI: https://doi.org/10.18174/540280 


\section{Table of contents}

$\begin{array}{lll}\text { Chapter } 1 & \text { General Introduction and Thesis Outline } & 7\end{array}$

Chapter 2 Breakthrough in Marine Invertebrate Cell Culture: Sponge Cells 19

Divide Rapidly in Improved Nutrient Medium

Sci Rep 9, 17321 (2019)

Chapter 3 Establishing the First Continuous Marine Sponge Cell Line

Chapter $4 \quad$ Transcriptome Analysis Provides Insights into How Marine Sponge 59

Geodia barretti Transitions from In Situ to In Vitro Conditions

Chapter 5 CRISPR/Cas12a-mediated Genome Editing in Geodia barretti 93

Sponge Cell Culture

Front Mar Sci 7, 599825 (2020)

Chapter 6 General Discussion: Dawn of a New Era in Sponge Biotechnology

Summary

References

Acknowledgements

Curriculum Vitae 
0 


\section{Chapter 1}

Introduction and Thesis Outline 


\subsection{Introduction}

Sponges are benthic animals that belong to the phylum Porifera, and fascinating creatures for many reasons. Sponges can inhabit both fresh- and marine water, but $99 \%$ of species live in marine environments. Marine sponges occur at all depths and in every ocean on the planet ${ }^{1}$. They constitute an important part of many marine ecosystems, and even form the basis for some ${ }^{2}$. First appearing in the fossil record about 600 million years ago, they are among the earliest and simplest animals ${ }^{3}$ and provide valuable insights into evolution of animals from single-celled eukaryotes ${ }^{4,5}$. Recently sponges have also emerged as a model of animal symbiosis with micro-organisms such as bacteria, microalgae and fungi ${ }^{6-}$ ${ }^{8}$. Last but not least, they are one of the richest natural sources of bioactive compounds with pharmaceutical and commercial potential ${ }^{9-13}$.

\subsection{Sponge physiology \& life cycle}

As filter-feeders, sponges take up nutrients from the large amounts of water they continuously pump through their body. Although sponges do not possess true tissues, they do have several different structures that each carry out a specific function. Water flows in through the ostia, small openings formed by tubular porocytes, that connect to the channels inside the sponge (Figure 1$)^{14}$. These channels carry the water through chambers lined with flagellated collar cells known as choanocytes that phagocytose bacteria and other micro-organisms ${ }^{15}$, and take up dissolved and particulate carbon sources ${ }^{16}$. Filtered water is then pumped into the central channel(s) and expelled through one or more larger openings, or oscula. Internal channels and choanocyte chambers are supported by the mesohyl, an extracellular matrix that may contain sponge collagen

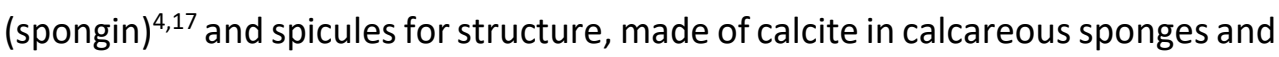
silica in all other sponges ${ }^{18}$. The mesohyl also contains cells, like archaeocytes, or sponge pluripotent stem cells ${ }^{17}$, spongin-producing (spongocytes) and spicule-producing cells (sclerocytes) ${ }^{18}$. Finally, the pinacoderm, an ectodermal layer of epithelial cells called pinacocytes, separates the sponge from its environment, helps it keep its shape and further supports internal structures ${ }^{18,19}$. 


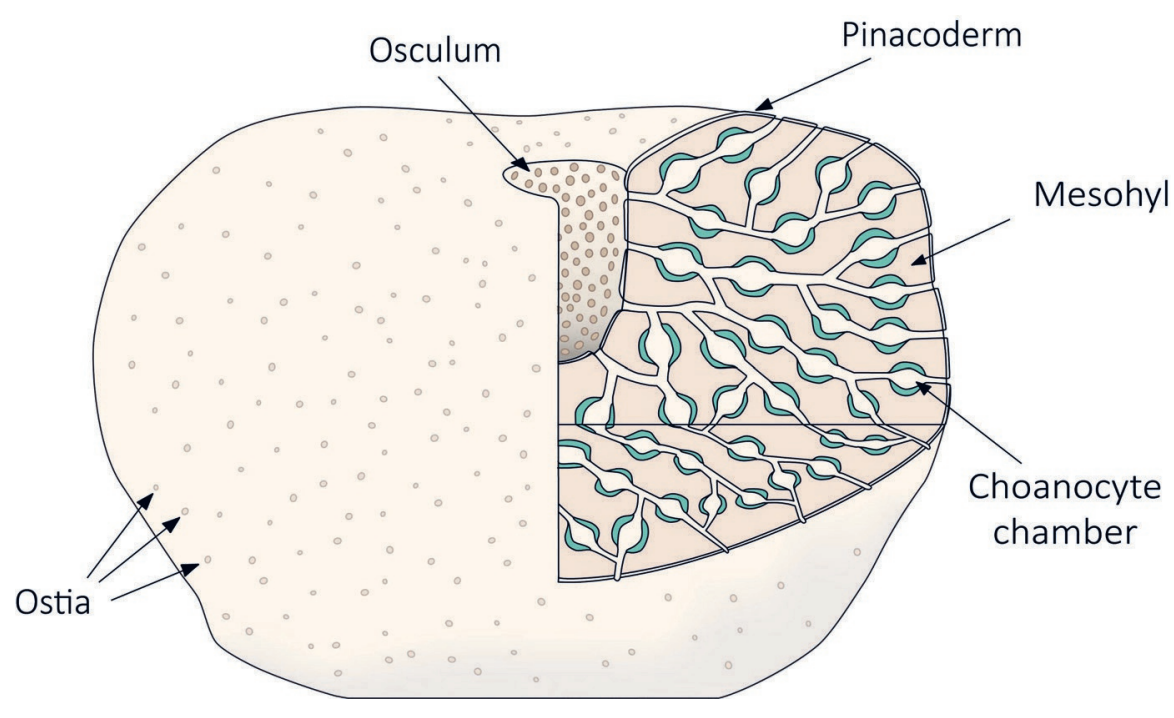

Figure 1. Schematic representation of an adult sponge. Choanocytes are shown in green.

The reproductive cycle in most sponges relies mainly on sexual reproduction ${ }^{20}$, although many sponges can also propagate asexually, and some are dependent on asexual reproduction ${ }^{21,22}$. Individuals of gonochoristic species each have a permanent gender, male or female. Other species can be sequential hermaphrodites that alternate between producing sperm and eggs, or simultaneous hermaphrodites that produce both at the same time $e^{20,23}$. Male sponges release their sperm in an event called 'spawning', while females either release their eggs to be fertilized and form an embryo in the water column (oviparous) or allow them to be fertilized and develop into an embryo inside their mesohyl before being released (viviparous). In both cases, the embryo will then become a free-swimming larva ${ }^{20,23}$. Sponge larvae drift along with the current for days, sometimes weeks, until they settle on the bottom, metamorphose, and grow to become a juvenile sponge. From this moment the sponge is sessile, meaning it will not move again during its life. Since sponges cannot move to find a mate, it is a great advantage ${ }^{21}$ that sponges can reproduce asexually in 3 different ways: budding, gemmulogenesis, and fragmentation ${ }^{20-23}$. Budding starts with a bulk of cells growing on the exterior of a sponge, until the bud is separated by constriction in the area connecting it to the parent sponge. 
Gemmules are small cell packages that are highly resistant to drought and physical damage. They develop inside the parent sponge and are only released when the surrounding sponge is severely damaged. Fragmentation happens when a part of the sponge is separated from the rest through physical damage from weather, animals or humans ${ }^{20,23}$. After dispersion by water currents the bud, gemmule or fragment settles and forms a small new sponge.

\subsection{Untapped biotechnological potential}

On the surface sponges may appear to have remained relatively simple, despite millions of years of evolution, but looks can be deceiving. Because they cannot move away from threats, sponges have evolved to defend themselves and interact with their environment by producing a large variety of complex chemicals (also known as compounds or secondary metabolites) with bioactive properties ${ }^{24}$ (Figure 2). For example, some compounds protect the sponge from harmful micro-organisms ${ }^{25-28}$, prevent obstruction of ostia through fouling ${ }^{29-31}$, successfully compete for space with other organisms ${ }^{32}$, and discourage predation by other animals ${ }^{28,33,34}$. The function of these secondary metabolites as a chemical defense is illustrated by the high incidence of bioactive compounds in species inhabiting densely populated ecosystems, such as coral reefs ${ }^{24}$. Sponges and sponge-derived microbes are the largest contributors to marine natural products discovered every year, with about $30 \%$ of these being of sponge origin $^{10-12}$. Many sponge-derived products have anticancer ${ }^{39,40}$, antibacterial ${ }^{41}$, antiviral $^{42}$ or antifungal ${ }^{43}$ activity, and could be potential drug candidates to combat disease in humans ${ }^{35-38}$. Avarol for example, produced by the Mediterranean sponge Dysidea avara, has a strong cytostatic effect on leukemic cells ${ }^{44,45}$. A recently discovered compound from a sponge symbiont inhibits growth and biofilm formation in several species of Staphylococcus bacteria ${ }^{46}$. Sponge compounds also have potential as commercial products, like nutritional supplements, cosmetics, molecular probes, enzymes and fine chemicals ${ }^{47}$. Each compound represents a potential multibillion dollar market ${ }^{48}$. 


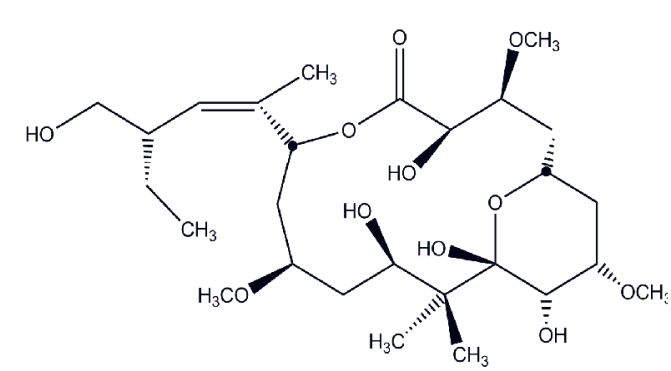

Barettin<smiles>Cc1c[nH]c2cc(Br)ccc12</smiles><smiles>CNC1=CCCCC1</smiles><smiles>CCNC(=O)[C@H](Cc1c(C(=O)O)[nH]c2ccccc12)NC</smiles>

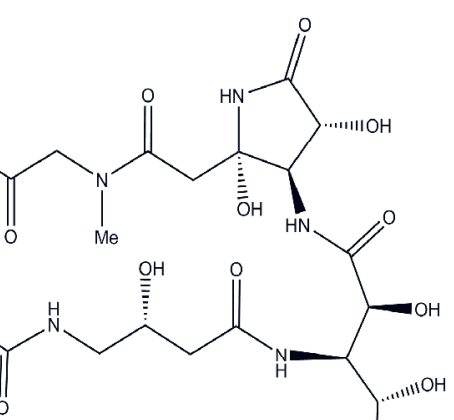

Figure 2. Compounds from sponges: Peloruside A (Mycale hentscheli), barettin (Geodia barretti) and Microsclerodermin A (Microscleroderma sp.)

Despite the wide variety of bioactive compounds discovered every year, currently there are only three sponge-derived drugs approved by the FDA ${ }^{40}$. Antitumor cytarabine and antiviral vidarabine originate in Tethya crypta, and antitumor eribulin mesylate is based on halichondrin $\mathrm{B}^{49}$, first discovered in Halichondria okadai ${ }^{50}$. Some other drug candidates are in various stages of clinical trials, but many face a serious supply problem ${ }^{32}$. Chemical synthesis is often not a viable option due to many compounds' complex structures (Figure 2 ), and metabolic pathways that produce the compounds are often equally complex and not well understood, ruling out transferring genes for microbial fermentation. Wild harvest to obtain a sufficient supply of compounds with therapeutic potential is not environmentally sustainable ${ }^{38}$. Aquaculture of sponges has some potential ${ }^{51,52}$, but provides limited control over environmental factors, is laborious and not feasible for all species because many compounds are naturally present at low concentrations ${ }^{32}$. Because of these issues, the enormous biotechnological potential of marine sponges remains largely 
untapped. Sponge cell lines could create enough biomass to produce the complex sponge-derived natural products at large scale in a controlled environment, and thus allow developing and testing novel drug leads. However, despite many efforts to establish in vitro cultures to study and produce these compounds ${ }^{47,53-60}$, there is still no sponge cell line available today.

\subsection{Cell culture \& challenges}

Culturing cells means keeping them alive outside of their usual environment, like tissue or blood. Cell cultures are the corner stone of biotechnological research and are used to identify and test potential new drugs ${ }^{61-63}$, produce such drugs at a large scale ${ }^{64}$, grow bone- or dental implants ${ }^{65-67}$, and determine how toxins, bacteria and viruses affect cells ${ }^{68}$, to name a few. The first step in establishing cell lines is obtaining a primary cell culture by transferring cells from the source to an artificial environment, like a culture vessel with liquid nutrient medium. Once the cells are growing, they are diluted before reaching their maximum cell density, or becoming completely confluent, meaning completely covering the bottom of the culture vessel. The secondary cultures grow and are diluted again before they reach a plateau. This process is called passaging and is repeated several times before the cultures are dubbed finite cell lines ${ }^{69}$. The first human cell line (HeLa) was created in 1951 by culturing cervical tumor cells from a woman named Henrietta Lacks ${ }^{70}$. Tumor cells are relatively easy to culture, as they are programmed to divide indefinitely, resulting in a continuous or immortal cell line. Both embryonic ${ }^{71}$ and adult ${ }^{72}$ stem cells are naturally immortal and have been used to establish continuous cell lines. Developing cell lines from healthy differentiated cells is more difficult and often requires a specifically tailored nutrient medium to support growth and prevent programmed cell death, or apoptosis ${ }^{73,74}$. Most animal cell lines need to adhere to a surface and/or other cells to survive, but some were adapted to grow while suspended in medium ${ }^{75-77}$. Finite cell lines can be immortalized by mimicking changes in the DNA that lead to cancer ${ }^{78-80}$, like overexpressing genes that stimulate cells to divide or disrupting genes that promote cell cycle arrest and apoptosis. 
Culturing sponge cells in vitro in any way, shape, or form has turned out to be quite challenging. Most studies have focused on monodispersed cell cultures, meaning single cell suspensions $s^{56,58,60}$. While primary cultures were quickly established ${ }^{53,54,81}$, cells generally did not divide $57,58,60$ and thus could not be passaged to become secondary cultures and eventually, cell lines. Analyzing how many cells were in different stages of the cell cycle showed that many cells stopped dividing and became apoptotic ${ }^{57}$. Sponge cells can also be cultured three-dimensionally, which provides contact with other cells and an extracellular matrix, which some hypothesize is needed for sponge cells to proliferate ${ }^{82-86}$. Dissociated sponge cells can reaggregate and after multiple stages of reorganizing, ultimately form a new, functional sponge. The first stage is forming primmorphs, organized spherical aggregates with an outer layer of skin-like pinacoderm ${ }^{87-89}$. Primmorphs can be cultured for months without ${ }^{87,90}$ or with low concentrations of ${ }^{91,92}$ added nutrients. Some cells in primmorphs of Suberites domuncula $^{89}$ and $H$. perleve ${ }^{93}$ synthesized DNA and proliferated. However, ultimately the biomass did not increase substantially, likely because while these cells started to grow, other cells went into apoptosis ${ }^{82,94}$.

Both types of cultures are often contaminated by prokaryotic and eukaryotic microbes ${ }^{58,87,93-95}$. Sponges have rich microbiomes and it is difficult, if not impossible, to take a sample of sponge tissue without including any microbes ${ }^{56}$. Many methods used to eliminate microbes were unsuccessful, such as adding antibiotics and -mycotics ${ }^{87}$, filtering cell suspensions, working aseptically, and separating cells of different sizes using density gradients ${ }^{58,81,96}$. Despite these precautions, contaminating microbes continued to plague sponge cell culture experiments. Another main issue was that little was known about the nutrients sponge cells need to proliferate ${ }^{58}$. Therefore, research focused on optimizing existing animal cell culture media for sponge cells to obtain more viable cell cultures $^{54,81,84,85,88,92,97-100}$ and this strategy yielded some promising results. Plant mitogen phytohemagglutinin (PHA) stimulated cells of two sponge species, Hymeniacidon heliophila ${ }^{81}$ and Axinella corrugata ${ }^{47,54}$, to divide and reach double the original cell concentration within 72 and 36 hours, respectively. 
Cultured archaeocytes isolated from $\mathrm{H}$. perleve increased in cell number by 2.5fold after 4 days ${ }^{101}$ in a previously developed improved nutrient medium ${ }^{98}$. However, these cultures could not keep growing because the totipotent archaeocytes differentiated into other cell types ${ }^{101}$. Adding $120 \mu \mathrm{M}$ dissolved silica to the culture medium increased the average area covered by Petrosia piciformis primmorphs from $0.1-0.2$ to $0.4-0.5 \mathrm{~mm}^{2}$, but higher concentrations did not increase the size further ${ }^{85}$. Recently, the amino acid concentrations of mammalian cell culture medium M199 where optimized for cells of Caribbean sponge Dysidea etheria, which caused the cells to be more metabolically active ${ }^{102}$. When optimized media did not lead to long-lasting sponge cell cultures, efforts were made to immortalize sponge cells by expressing oncogenes such as ras genes ${ }^{58}$ and human telomerase reverse transcriptase (hTERT). While hTERT was successfully expressed in $A$. corrugata cells, these cells were not immortalized $^{59}$. Another method to immortalize cells is by fusing them with other cells, creating a hybrid cell line. Sponge cells of different species were fused successfully ${ }^{55,58}$, and Haliclona sp. cells fused with insect cells ${ }^{59}$, but none of the hybrid cells started growing. Despite all the different approaches described here, and more, the first sponge cell line still needs to be reported.

\subsection{Thesis outline}

Attempts to culture sponge cells in vitro, regardless of the approach, are thwarted by 3 major obstacles: not knowing what nutrients sponge cells require to grow and maintain themselves, difficulties containing contamination by microbes, and being unable to consistently subculture dividing cells once they are obtained. The research presented in this thesis focused on how to overcome these obstacles and develop the first marine sponge cell line.

In Chapter 2 we develop culture methods for marine sponge cells in an amino acid-optimized medium (M1). This medium was originally optimized based on metabolic activity in the Caribbean sponge Dysidea etheria but did not stimulate the cells to divide ${ }^{103}$. However, when we tested how other sponge species responded to the medium, cells of many species grew rapidly. Several Geodia 
spp. including G. barretti showed the strongest response and the least individual variation. Cells could be subcultured multiple times and reach a maximum of 7 population doublings. This was a breakthrough in sponge biotechnology that opened many possibilities, some of which we explored in the next chapters.

Chapter 3 focuses on further improving conditions to stimulate $G$. barretti cells to proliferate, by culturing cells in OpM1, an improved medium based on $\mathrm{M} 1$, in which other nutrients such as lipids, fetal bovine serum, growth factors, vitamins and other components were optimized for D. etheria (Munroe et al. in prep). $G$. barretti cells grew faster and to a higher cell density in this medium than in M1 medium. The largest difference was in the longevity of the cultures: cells from 3 individuals of $G$. barretti continued to divide until nearly 100 population doublings, 20 times as many as cells from the same 3 individuals could reach in M1 medium. The first continuous sponge cell line was established, bringing us one step closer to marine sponge cells producing biopharmaceuticals in vitro.

In Chapter 4 we study how $G$. barretti cells react to OpM1 medium, by comparing how genes are expressed in the cells' physiological state in the intact sponge, after the cells have been dissociated and cryopreserved, and when cells are cultured in OpM1. Studying which genes are differentially expressed and which gene sets are enriched in these states provides new insight into the molecular biology and physiology of sponges and their cells, for example about their metabolism, how they respond to stress, and recover from it, and how they regulate cell division and apoptosis. This knowledge can be used when choosing target genes to immortalize cells, designing improved media, or optimizing sponges' metabolism to synthesize valuable compounds.

Establishing molecular tools is essential to develop a continuous cell line, by disrupting tumor-suppressor genes and overexpressing oncogenes to immortalize cells. Additionally, metabolic pathways can be altered to identify enzymes that are essential for synthesizing desired products and can be targeted to influence which products are synthesized at what rate. In Chapter 5 we show for the first time that CRISPR-Cpf1 can be used to edit the genome of sponge cells in vitro. We demonstrate that preformed CRISPR-Cpf1 ribonucleoprotein 
(RNP) complexes can be delivered into G. barretti cells using lipofection. Once there, the RNPs can cut the genome of $G$. barretti, and a sequence can be inserted through homologous recombination, either disrupting the target gene or introducing a gene to overexpress it.

In Chapter 6 we discuss the future of sponge biotechnology. In the work presented in this thesis, we took important steps forward. We showed that sponge cells of multiple species can divide rapidly in M1 medium (Munroe et al., 2019) and cultures could be maintained for multiple weeks. We developed the first marine sponge cell line by culturing $G$. barretti cells in the improved OpM1 medium. Finally, we provide the first evidence of CRISPR-Cas genome editing in sponge cells, taking the first step to start developing the molecular tool box required to improve strains using genetic modification. These advancements have cleared the way for new lines of research. Now is an exciting time in marine sponge biotechnology, so what's next? 


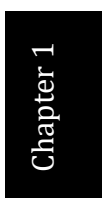


0 


\section{Chapter 2}

\section{Breakthrough in Marine Invertebrate Cell Culture:}

\section{Sponge Cells Divide Rapidly in Improved Nutrient \\ Medium}

This chapter is published as:

Conkling, M., Hesp, K., Munroe, S. et al. Breakthrough in Marine Invertebrate Cell Culture: Sponge Cells Divide Rapidly in Improved Nutrient Medium. Sci Rep 9, 17321 (2019) doi:10.1038/s41598-01953643-y 


\section{Abstract}

Sponges (Phylum Porifera) are among the oldest Metazoa and considered critical to understanding animal evolution and development. They are also the most prolific source of marine-derived chemicals with pharmaceutical relevance. Cell lines are important tools for research in many disciplines, and have been established for many organisms, including freshwater and terrestrial invertebrates. Despite many efforts over multiple decades, there are still no cell lines for marine invertebrates. In this study, we report a breakthrough: we demonstrate that an amino acid-optimized nutrient medium stimulates rapid cell division in 9 sponge species. The fastest dividing cells doubled in less than 1 hour. Cultures of 3 species were subcultured from 3 to 5 times, with an average of 5.99 population doublings after subculturing, and a lifespan from 21 to 35 days. Our results form the basis for developing marine invertebrate cell models to better understand early animal evolution, determine the role of secondary metabolites, and predict the impact of climate change to coral reef community ecology. Furthermore, sponge cell lines can be used to scale-up production of sponge-derived chemicals for clinical trials and develop new drugs to combat cancer and other diseases. 


\subsection{Introduction}

Sponges (Phylum Porifera) are key components of many benthic marine ecosystems. There are more than 9,000 described species that occur worldwide, from the intertidal to the deep sea ${ }^{104}$. Among the oldest metazoans, sponges have evolved a variety of strategies to adapt to different environments. Because they are sessile as adults, they have evolved sophisticated chemical systems for communication, defense from predators, antifoulants to prevent other organisms from growing over them, and to prevent infection from microbes filtered out of the water ${ }^{105,106}$. These chemicals interact with molecules that have been conserved throughout evolutionary history and are involved in human disease processes, for example, cell cycling ${ }^{57}$, immune and inflammatory responses ${ }^{107}$, and calcium and sodium regulation ${ }^{108,109}$.

Vertebrate, insect, and plant cell lines are important tools for research in many disciplines, including human health, evolutionary and developmental biology, agriculture, and toxicology. Although cell lines have been established for freshwater and terrestrial invertebrates (e.g., Hydra, Caenorhabditis), and longterm (>1 month) primary cultures have been reported for cells derived from tissues of the cnidarian Anemonia viridis and the shrimp Penaeus ${ }^{60,110}$, attempts to establish cell lines from marine invertebrates have been unsuccessful ${ }^{58,60,111}$. Marine sponges, including some of the species in this study, are the source of thousands of novel chemicals with pharmaceutically relevant properties ${ }^{94,112,113}$. Supply of these chemicals is a bottleneck to development of sponge-derived drug leads: wild harvest is not ecologically sustainable, and chemical synthesis is challenging due to the complexity of many of the bioactive chemical compounds. In vitro production has been proposed as an option, but the lack of permanent sponge cell lines makes this option unfeasible.

Sponge cell lines could be used as models to understand the role of secondary metabolites in sponges, to use this information to develop new models for drug discovery, and to scale-up production of sponge-derived bioactive compounds for novel medicines. Cell lines of common reef sponges could also be used to 
quantify the effects of climate change (ocean warming and acidification) on uptake of dissolved organic material (DOM), a major component of the "sponge loop hypothesis" of carbon cycling and to test the hypothesis that coral reefs could become sponge reefs as climate changes ${ }^{114}$.

To date, primary cultures have been established from dissociated and cryopreserved cells of several sponge species ${ }^{57,96,102,115}$; optimized nutrient media have been developed 97, 98, 103,115, 116 ; cell division has been stimulated with growth factors and mitogens ${ }^{54,81}$ (Munroe et al. in prep); transient expression of immortalizing genes has been obtained ${ }^{117}$; somatic cell hybridization has been demonstrated ${ }^{55}$; and methods for three-dimensional culture in hydrogels have been established ${ }^{118,119}$ (Munroe et al. in prep). These improvements in sponge cell culture were sporadic and incremental, and resulted only in a limited number of cell divisions. In this study, we report a breakthrough in marine invertebrate (sponge) cell culture: using an optimized nutrient medium ${ }^{103}$, a substantial increase in both the rate and number of cell divisions has been accomplished for the first time.

\subsection{Materials \& Methods}

\subsubsection{Sample collection}

Individuals of twelve sponge species (Class Demospongiae) from seven orders and eight families were collected for this study. Amphimedon erina (Order Haplosclerida, Family Niphatidae), Cliona varians (Order Clionaida, Family Clionaidae), Dysidea etheria (Order Dictyoceratida, Family Dysideidae), Geodia sp. (Order Tetractinellida, Family Geodiidae), an unidentified species of Spongiidae (Order Dictyoceratida), and Tedania ignis (Order Poecilosclerida, Family Tedaniidae) were collected from Atlantic coastal waters off Summerland Key, Florida $\left(24^{\circ} 39^{\prime} 36.9^{\prime \prime} \mathrm{N} 81^{\circ} 27^{\prime} 18.0^{\prime \prime} \mathrm{W}\right)$ at a depth of approximately one to two meters, from sandy bottom grass flats and mangrove roots. Amphimedon compressa (Order Haplosclerida, Family Niphatidae), Aplysina fulva (Order Verongiida, Family Aplysinidae), Axinella corrugata (Order Axinellida, Family Axinellidae), Geodia neptuni (Order Tetractinellida, Family Geodiidae) and 
Niphates erecta (Order Haplosclerida, Family Niphatidae) were collected from a deeper ( 20 meters) reef site off Looe Key, Florida $\left(24^{\circ} 32^{\prime} 44.6^{\prime \prime} \mathrm{N} 81^{\circ} 24^{\prime} 21.4^{\prime \prime} \mathrm{W}\right)$, characterized by hard bottom with dense sponge, coral and algae cover. Geodia barretti (Order Tetractinellida, Family Geodiidae) individuals were collected in a single trawl at a depth of $~ 500$ meters in a fjord $\left(59^{\circ} 58.8^{\prime \prime} \mathrm{N} 5^{\circ} 22.4^{\prime \prime} \mathrm{E}\right)$ close to Bergen, Norway. Collections from Florida were authorized under permit \#FKNMS-2014-070 from the National Oceanic and Atmospheric Administration, Office of National Marine Sanctuaries, and Special Activity License SAL-14-1588SR from the Florida Fish and Wildlife Conservation Commission.

\subsubsection{Sample identification}

Taxonomic identification of the sponges was confirmed by evaluation of morphological characters: morphology, color, surface texture, and microscopic analysis of the skeleton. Geodia sp. (reported as Geodia vosmaeri ${ }^{120}$ ) is in the process of being re-described (Cardenas, personal communication), but it is an easily recognized species in shallow water grass flats off the coast of south Florida.

\subsubsection{Dissociation and cryopreservation}

Cells from all studied species were dissociated and cryopreserved immediately after sampling using previously established methods ${ }^{47,54,102}$. Sponges were cleaned of debris and rinsed in seawater filtered through a sterile $0.22 \mu \mathrm{m}$ filter (FSW). Cells were dissociated by squeezing fragments of sponge through sterile gauze (grade 16 mesh size for G. barretti [B. Braun Medical] and grade 10 mesh size for all other species [Fisherbrand]) and filtering the cell suspension through a cell strainer ( $40 \mu \mathrm{m}$ [Greiner Bio-One] for G. barretti and $70 \mu \mathrm{m}$ [Fisherbrand]) for all other species) to eliminate debris, cell aggregates and spicules. Cells were washed twice by centrifugation at $300 \times \mathrm{g}$ for 5 minutes and resuspended in FSW. Cell concentrations of $G$. barretti were microscopically counted using disposable hemocytometers ( $\mathrm{C}$-chip ${ }^{\mathrm{TM}}$, Neubauer improved); cells from all other species were automatically counted using the Countess II FL Automated Cell Counter (Thermo Fisher). Dissociated cells from all species were cryopreserved at a cell 
concentration of approximately $1.00 \mathrm{E}+08 \mathrm{cells} / \mathrm{mL}$ in a cryoprotectant solution (10\% dimethyl sulfoxide (DMSO) and 10\% fetal bovine serum (FBS) in FSW $)^{54,86}$, ${ }^{102}$. Cells were pipetted $(1 \mathrm{~mL}$ ) into cryogenic vials (Fisherbrand), the vials were placed in Nalgene ${ }^{\circledast} \mathrm{Mr}$. Frosty freezing containers and cooled to $-80^{\circ} \mathrm{C}$ at a steady rate of $1{ }^{\circ} \mathrm{C}$ per minute.

\subsubsection{Media preparation}

Artificial seawater (ASW), modified from Zhang et al. (2004), was prepared by dissolving salts into filter sterilized distilled water (DIW) and then autoclaving at $121^{\circ} \mathrm{C}$ for $25 \mathrm{~min}^{102,103}$. Medium 199 (Sigma Aldrich, M3769) was prepared according to the manufacturer's protocol. M1 medium was prepared by dissolving Medium 199 powder (Sigma Aldrich, M3769) in distilled water (DIW). Salts were added in concentrations to approximate the $\mathrm{pH}$ (medium: 7.9; ocean: 8.1) and salinity (medium: $33.5 \mathrm{ppt}$; ocean: $35 \mathrm{ppt}$ ) of seawater (Table S1). Next, amino acids were added in the concentrations that were optimized for in vitro culture of the sponge $D$. etheria ${ }^{103}$ (Table S1). Both M1 and M199 were supplemented with rifampicin (Sigma Aldrich, R3501) and amphotericin B (Sigma Aldrich, A2411) to control bacterial and fungal contamination, respectively.

\subsubsection{Establishment of primary cultures}

For the first set of experiments, primary cultures of twelve species (one individual of each species) were evaluated to determine which species would proliferate in three different media (Table S1): artificial seawater (ASW), Medium 199 (M199) ${ }^{54} 24$ and M1 medium ${ }^{103}$. Cryopreserved cells were thawed rapidly in a $50{ }^{\circ} \mathrm{C}$ water bath to minimize ice crystal damage to the cells $s^{47,54}$. The cell suspension was rinsed twice by centrifugation at $4000 \mathrm{rpm}$ for 5 minutes and resuspended in ASW. Cell number was measured automatically using the Countess II FL Automated Cell Counter (Thermo Fisher) for all time points for all species except $G$. barretti, for which cell concentrations were counted microscopically using a disposable hemocytometer (C-chip, Neubauer improved). Prior to counting the cells, the cell suspension was gently pipetted to disperse aggregates. Cell aggregation was generally not an issue, however, if 
aggregation prevented accurate cell counts, the cells were resuspended in calcium- and magnesium-free artificial seawater $(\mathrm{CMF})^{57}$ prior to counting. Cell concentrations at time point zero were calculated by counting the cell suspension and then resuspending in either ASW, M199, or M1 to the desired concentration. Samples were cultured in 24-well plates (Falcon): for the first two experiments (to determine if cells were dividing and to further evaluate cell proliferation in the 6 selected species), cells were incubated for four to six days at $\sim 22^{\circ} \mathrm{C}$ for all species except $\mathrm{G}$. barretti, which was incubated at $4{ }^{\circ} \mathrm{C}$. These temperatures are within the range of ambient seawater temperatures for the Florida and Norway sites, respectively. To further characterize cell division and to determine the number of times the cells could be subcultured, the three Geodia species were incubated at both $\sim 22^{\circ} \mathrm{C}$ and $4{ }^{\circ} \mathrm{C}$.

\subsubsection{Growth characterization}

Growth curves in M1 were determined for G. barretti, Geodia sp., and G. neptuni ( $n=3$ specimens for each species). Specimens (=individuals) were prepared in triplicate, in 96-well plates (Falcon), for each individual and time point. All samples were incubated at $\sim 22^{\circ} \mathrm{C}$ and at $4{ }^{\circ} \mathrm{C}$. Cell number was measured hourly for 12 hours, every 12 hours for 48 hours and every 24 hours for 120 hours ( 5 days) for all cultures at $22^{\circ} \mathrm{C}$, as well as $\mathrm{G}$. barretti cultures at $4{ }^{\circ} \mathrm{C}$. Cultures of Geodia sp. and $G$. neptuni at $4{ }^{\circ} \mathrm{C}$ were measured hourly for 24 hours and every 24 hours for 120 hours. Three seeding densities $(5.0 \mathrm{E}+05,1.0 \mathrm{E}+06$, and 5.0E+06 cells $/ \mathrm{mL}$ ) were evaluated to determine the effect of inoculation density on final cell densities (unpublished data). The optimal seeding density (i.e., the inoculation density that resulted in the greatest increase in cell number) was 3.0E+06 cells $/ \mathrm{mL}$ for $\mathrm{G}$. barretti and 5.0E+06 cells $/ \mathrm{mL}$ for Geodia sp. and G. neptuni. Cells were automatically counted using the Countess II FL Automated Cell Counter (Thermo Fisher) for Geodia sp. and G. neptuni, and manually counted for $G$. barretti using disposable hemocytometers (C-chip, Neubauer improved) for all time points except for $G$. barretti at time point zero, which was calculated by counting the cell suspension, and then resuspending in $M 1$ at the desired concentration. 


\subsubsection{Passaging cells}

Cultures were passaged in $\mathrm{M} 1$ using three individuals per species, in triplicate, in 24-well plates (Falcon). All three species were cultured at both $\sim 22^{\circ} \mathrm{C}$ and $4{ }^{\circ} \mathrm{C}$. The seeding density was $3.0 \mathrm{E}+06$ cells $/ \mathrm{mL}$ for $\mathrm{G}$. barretti, and $5.0 \mathrm{E}+06$ cells $/ \mathrm{mL}$ for Geodia sp. and G. neptuni. Passaging was performed by resuspending the cells by pipetting, determining the cell concentration and subsequently diluting the cells, by either splitting the culture (e.g., 1:2 ratio) or diluting back to the seeding density. Cultures were passaged until the cells stopped dividing in order to determine the lifespan of the cell lines for each species. Cell number was measured automatically using the Countess II FL Automated Cell Counter (Thermo Fisher) for Geodia sp. and G. neptuni. Geodia barretti cells were counted microscopically using disposable hemocytometers (C-chip, Neubauer improved) for all time points except for time point zero which was calculated by counting the cell suspension and then diluting to the desired concentration. Cultures of all three species were monitored microscopically (EVOS FL Auto imaging system, Invitrogen).

\subsubsection{Sponge cell verification}

To confirm that the cells in culture were from the three species of Geodia, $18 \mathrm{~S}$ rRNA gene amplicon sequencing and eukaryotic community profiling was performed on cultures of all three species, both before (time point zero for all three species) and after passaging (one passage, time point 48 hours, $\mathrm{T}=22^{\circ} \mathrm{C}$ for $G$. neptuni and Geodia sp.; four passages, time point 28 days, $T=4^{\circ} \mathrm{C}$ for $G$. barretti). Cell pellets (approximately $1.0 \mathrm{E}+08$ cells) of each species were stored at $-20^{\circ} \mathrm{C}$. Genomic DNA extraction, $18 \mathrm{~S}$ rRNA gene amplification through polymerase chain reaction (PCR) and Illumina MiSeq sequencing and sequence analysis were performed by RTL Genomics (Lubbock, Texas, USA). A High Pure PCR Template Preparation Kit (Roche Life Science, Basel, Switzerland) was used to extract genomic DNA following the manufacturer's protocol, with one exception: after addition of binding buffer and proteinase $\mathrm{K}$, the samples were incubated at $70{ }^{\circ} \mathrm{C}$ for a prolonged period ( 35 minutes) to increase DNA yield. Fungal primers that were previously used for eukaryotic community analysis of 
a sponge holobiont ${ }^{121}$ were used to amplify an approximately 350 base pair long region of the eukaryotic small-subunit rRNA gene, including the V7 and V8 hypervariable regions in a two-step process. The forward primer was constructed with $\left(5^{\prime}-3^{\prime}\right)$ Illumina i5 sequencing primer (TCGTCGGCAGCGTCAGATGTGTATAAGAGACAG) and the FF390 primer (CGATAACGAACGAGACCT) $^{122}$. The reverse primer was constructed with $\left(5^{\prime}-3^{\prime}\right)$ Illumina i7 sequencing primer (GTCTCGTGGGCTCGGAGATGTGTATAAGAGACAG) and the FR1 primer (ANCCATTCAATCGGTANT) ${ }^{122}$. Amplifications were performed in $25 \mu \mathrm{L}$ reactions with Qiagen HotStarTaq master mix (Qiagen Inc., Valencia, California), $1 \mu \mathrm{L}$ of each $5 \mu \mathrm{M}$ primer, and $1 \mu \mathrm{L}$ of template. Reactions were performed on $\mathrm{ABI}$ Veriti thermocyclers (Applied Biosystems, Carlsbad, California) under the following thermal profile: $95^{\circ} \mathrm{C}$ for 5 minutes, then 30 cycles of $95^{\circ} \mathrm{C}$ for 30 seconds, $50^{\circ} \mathrm{C}$ for 45 seconds, and $72{ }^{\circ} \mathrm{C}$ for 1 minute, followed by a final extension of $72^{\circ} \mathrm{C}$ for 10 minutes, and a $4{ }^{\circ} \mathrm{C}$ hold. Products from the first stage amplification were added to a second PCR based on qualitatively determined concentrations. Primers for the second PCR were designed based on the Illumina Nextera PCR primers: ForwardAATGATACGGCGACCACCGAGATCTACAC [i5index]TCGTCGGCAGCGTC and Reverse-CAAGC AGAAGACGGCATACGAGAT [i7index]GTCTCGTGGGCTCGG. The second stage amplification was run with the following thermal profile: $95^{\circ} \mathrm{C}$ for 5 minutes, then 10 cycles of $94^{\circ} \mathrm{C}$ for 30 seconds, $54{ }^{\circ} \mathrm{C}$ for 40 seconds, and $72{ }^{\circ} \mathrm{C}$ for 1 minute, followed by a final extension of $72^{\circ} \mathrm{C}$ for 10 minutes and a $4{ }^{\circ} \mathrm{C}$ hold. Sequence data were analyzed using the in-house data analysis pipeline of RTL Genomics (version 2.3.1).

\subsection{Results}

\subsubsection{An amino acid-optimized nutrient medium stimulates rapid cell division in primary cell cultures of marine sponges}

We cultured cells of 12 sponge species in three different media: artificial seawater (ASW), Medium 199 (M199), and M1 (Figure 1). As predicted from prior research ${ }^{57,96,115}$, the number of cells cultured in ASW either remained the same 


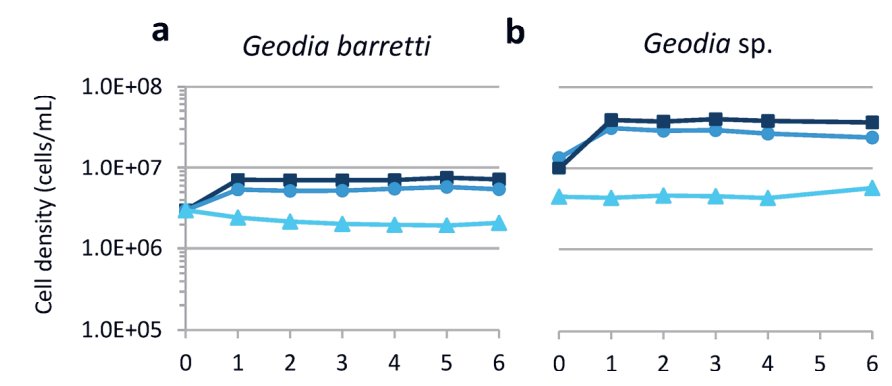

C Geodia neptuni
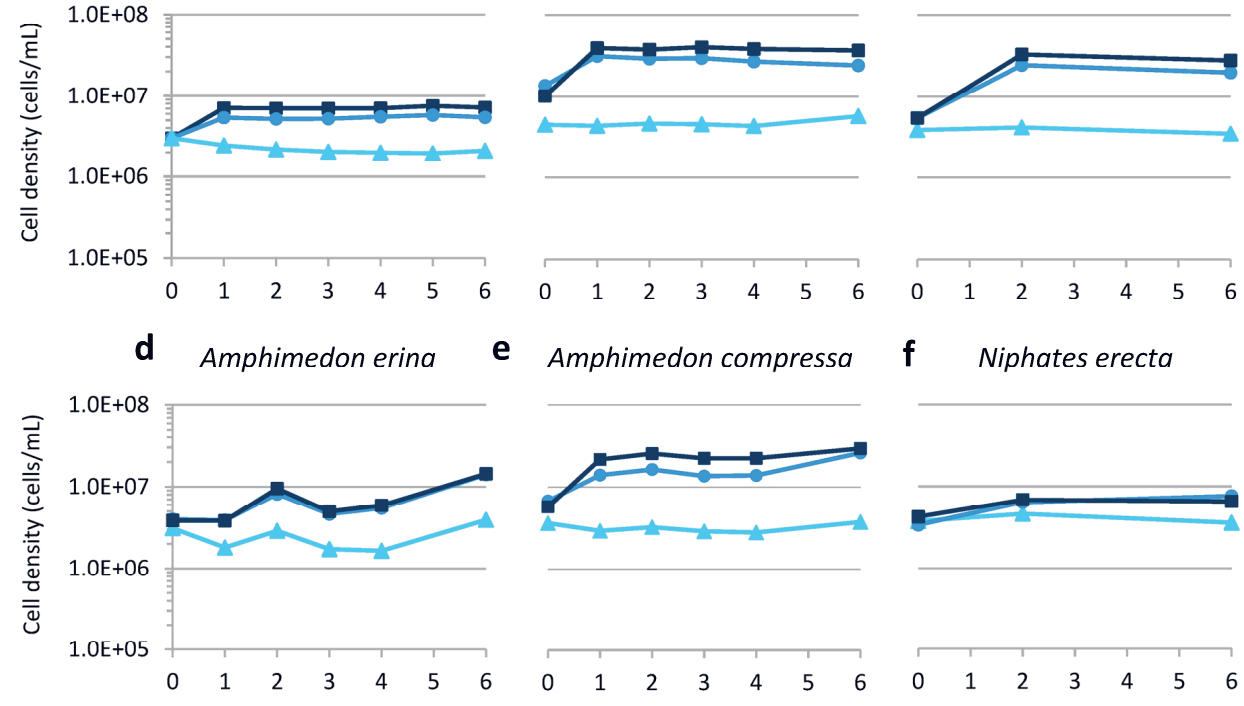

e Amphimedon compressa

f Niphates erecta
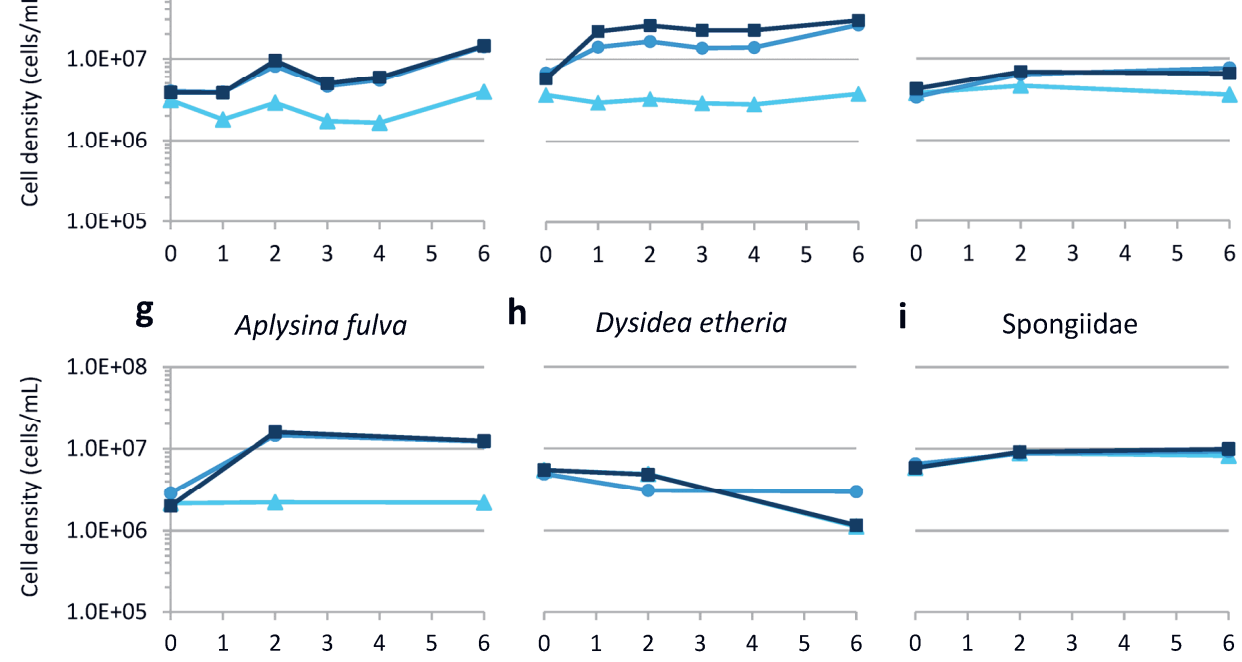

h Dysidea etheria

i Spongiidae
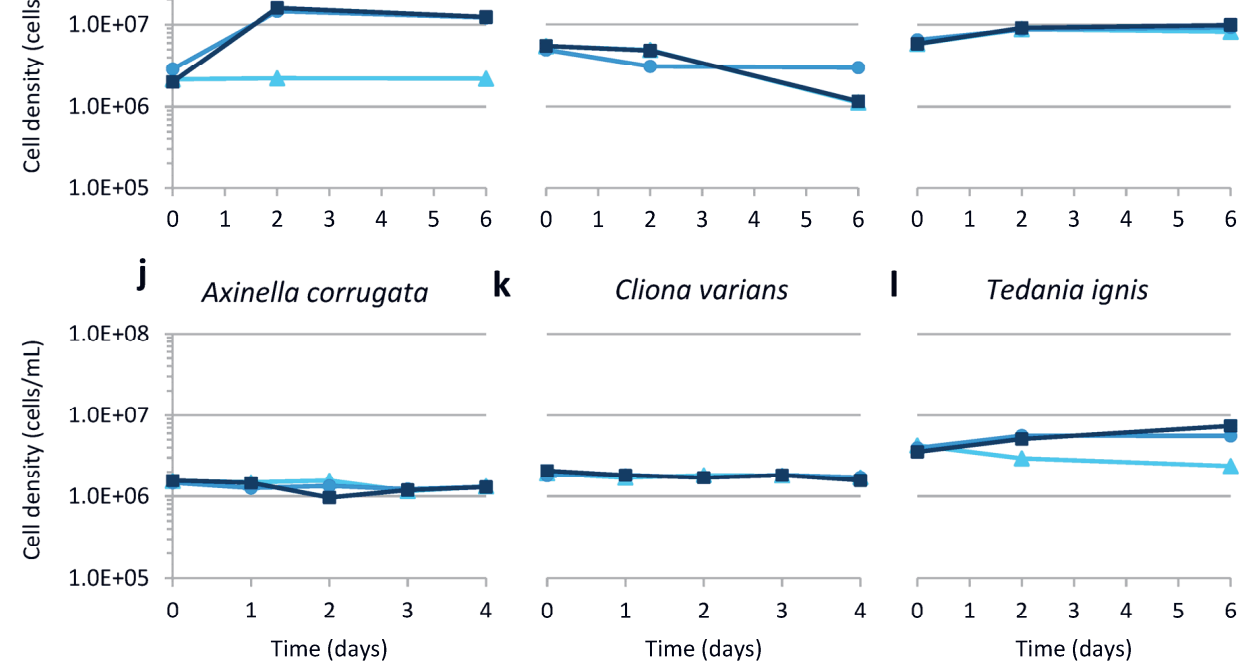

k

Cliona varians

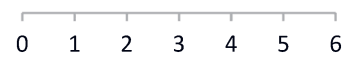

(1)
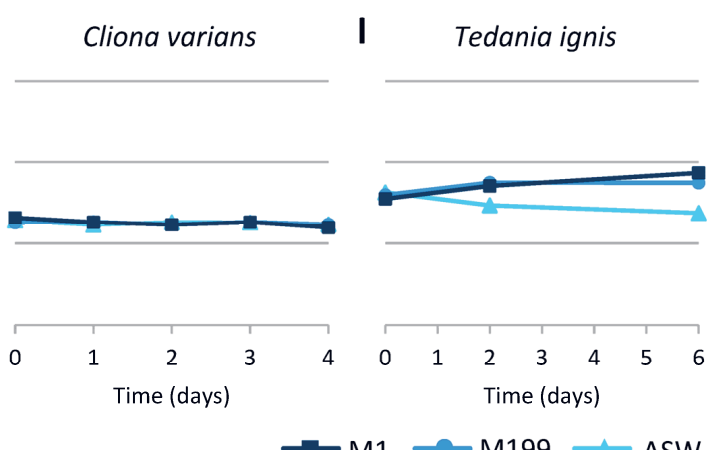

Figure 1. Primary cultures of 12 sponge species in three different media: artificial seawater (ASW), Medium 199 (M199) and M1 medium. (a) Geodia barretti, (b). Geodia sp., (c). Geodia neptuni, (d). Amphimedon erina, (e). Amphimedon compressa, (f). Niphates erecta, (g). Aplysina fulva, (h). Dysidea etheria, (i). Spongiidae, (j). Axinella corrugata, (k). Cliona varians, (l). Tedania ignis. Only 1 individual of each species was tested, however, the results are the average of 3 technical replicates $(n=3) \pm$ standard deviation. 
or decreased, except for an unidentified species of Spongiidae (Figure 1i). Although the pattern of cell number increase and decrease in ASW for Amphimedon erina parallels the pattern for M199 and M1 after the initial decrease in ASW (Figure 1d), there was only a small increase in cell number in ASW ( 25\%) compared with nearly a three-fold increase in cell number in M199 and $M 1$ after day 1 . Within two days of incubation in $M 1$, cell numbers increased for each of the following species: Geodia barretti, Geodia sp., G. neptuni, A. erina, Amphimedon compressa, Niphates erecta, Aplysina fulva, the unidentified species of Spongiidae, and Tedania ignis (Figure 1a-g,i,l). Of these, G. barretti, Geodia sp., G. neptuni, A. erina, A. compressa, and A. fulva had the largest increase in cell number in $\mathrm{M} 1$, with between 1.5 and 3 population doublings (Figure 1a-e,g). Cell numbers also increased in M199 for the same six species, although either lower than ( $G$. barretti, Geodia sp., G. neptuni, and A. compressa) or equal to ( $A$. erina and $A$. fulva) the increase in cell numbers in $M 1$.

For three species (D. etheria, $A$. corrugata, and $C$. varians), there was no increase in cell number in any medium (Figure $1 \mathrm{~h}, \mathrm{j}, \mathrm{k}$ ). The medium in cultures of each individual of each species with increases in cell number changed color from pale orange to dark grey, and the cells appeared microscopically to have dark inclusions (unpublished data). These changes were observed as soon as cultures increased in cell number and became increasingly darker as the cell density increased. The color change is not associated with a change in $\mathrm{pH}$ of the culture medium (spent medium $\mathrm{pH}$ : 7.8). Research is in progress to determine the cause of the color change and to characterize the cell inclusions.

\subsubsection{Individual (intraspecific) variation must be factored into selection of source material}

To evaluate individual (intraspecific) variation, we focused subsequent studies on the six species with the largest increase in cell density in M1: G. barretti, Geodia sp., G. neptuni, A. compressa, A. erina, and A. fulva (Figure 1). For these species, cells from multiple individuals were cultivated in triplicate for 48 hours in M1 medium. There was little individual (intraspecific) variation in final cell 
density for each of the three species of Geodia cultured for 48 hours (Figure 2ac). Conversely, individuals of $A$. erina, $A$. compressa, and A. fulva had individual (intraspecific) variation in final cell density (Figure $2 \mathrm{~d}-\mathrm{f}$ ).
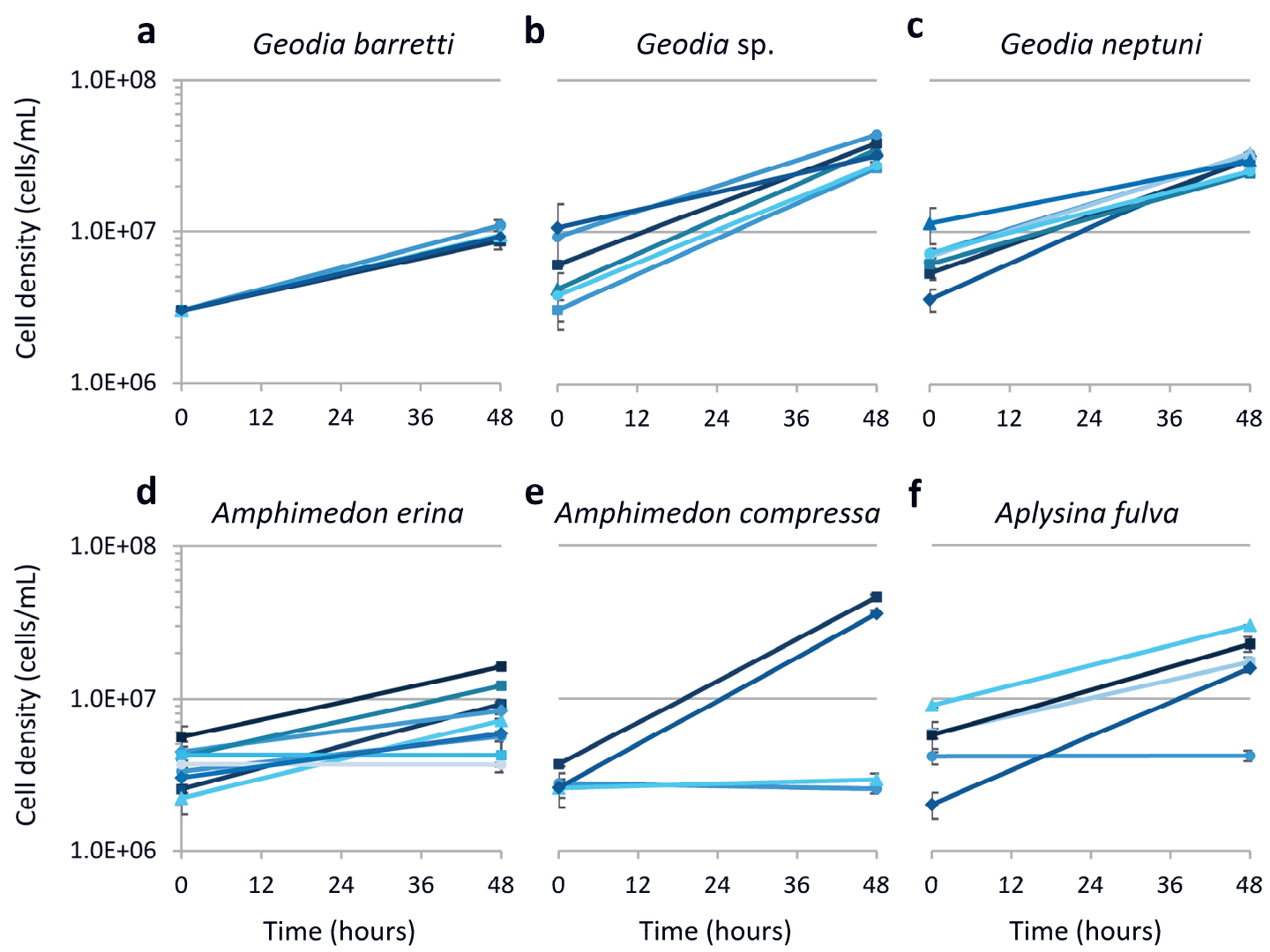

Figure 2. Primary cultures of the 6 sponge species selected for further studies in M1 medium. a. G. barretti $(n=4)$, b. G. cf. gibberosa $(n=6)$, c. G. neptuni $(n=7)$, d. A. erina $(n=9)$, e. A. compressa $(n=4), f . A$. fulva $(n=5)$. Each line represents an individual. Error bars indicate standard deviation from the average of 3 technical replicates. 


\subsubsection{Marine sponge cells are capable of rapid cell division}

In this study, we selected three species of Geodia because there was little intraspecific variation when cultured in M1 medium. In addition, G. barretti is the source of compounds with anti-inflammatory activity ${ }^{123}$. Cell density of $G$. barretti, Geodia sp., and G. neptuni was measured with a finer time resolution to understand the dynamics of cell division for each species (Figure 3 ) and to identify when the cultures were near the end of exponential growth and, therefore, ready to subculture/passage. Growth curves were analyzed for all three species at both $22^{\circ} \mathrm{C}$ (Figure 3a-c), the temperature at which M1 medium was optimized, and $4{ }^{\circ} \mathrm{C}$ (Figure $3 \mathrm{~d}-\mathrm{f}$ ). These temperatures were chosen because Geodia sp. occurs on shallow grass flats ( $\sim 2$ meters) and G. neptuni occurs on shallow reefs ( 20 meters) off Summerland Key, FL, USA, with sea surface temperatures ranging from $21.5^{\circ} \mathrm{C}$ to $30.5^{\circ} \mathrm{C}$, and G. barretti occurs in deeper water ( $\sim 500$ meters) in Norwegian fjords, with sea surface temperatures ranging from $5.5^{\circ} \mathrm{C}$ to $15.5^{\circ} \mathrm{C}$. Rapid cell division was observed in all Geodia species (Figure 3a-f), although the number of population doublings $\left(N_{d}\right)$ varied between species and incubation temperatures (Table 1).

Geodia barretti cultures did not reach the same density as the other two species. There was little individual variation in peak cell density for $G$. barretti when cultured at $4{ }^{\circ} \mathrm{C}$, with an average of $8.54 \mathrm{E}+06$ cells $/ \mathrm{mL}$ (Figure 3a). When $\mathrm{G}$. barretti cells were cultured at $22^{\circ} \mathrm{C}$, two individuals reached peak densities of $1.67 \mathrm{E}+07$ cells $/ \mathrm{mL}$ and $1.73 \mathrm{E}+07$ cells $/ \mathrm{mL}$ (Figure 3a). One individual at $22^{\circ} \mathrm{C}$ had a lower peak density of $1.11 \mathrm{E}+07$ cells $/ \mathrm{mL}$ (Figure 3a). Two individuals of Geodia sp. cultured at $22^{\circ} \mathrm{C}$ reached a peak density of $6.03 \mathrm{E}+07$ cells $/ \mathrm{mL}$ and $6.08 \mathrm{E}+07 \mathrm{cell} / \mathrm{s} / \mathrm{mL}$ within 12 hours (Figure $3 \mathrm{~b}$ ). The third individual cultured at $22^{\circ} \mathrm{C}$ a significantly higher peak density $(8.38 \mathrm{E}+07$ cells $/ \mathrm{mL})$ within 9 hours (Figure 3b). Different individual responses were observed for Geodia sp. cells cultured at $4{ }^{\circ} \mathrm{C}$. Two individuals cultured at $4{ }^{\circ} \mathrm{C}$ reached a peak density of $4.29 \mathrm{E}+07$ cells $/ \mathrm{mL}$ and $4.71 \mathrm{E}+07 \mathrm{cell} / \mathrm{mL}$ (Figure 3e). At $4{ }^{\circ} \mathrm{C}$, one individual reached a higher peak density of $5.61 \mathrm{E}+07$ cells $/ \mathrm{mL}$ within 24 hours (Figure 3e); this was not the same individual with the highest density at $22^{\circ} \mathrm{C}$. Cultures of all 

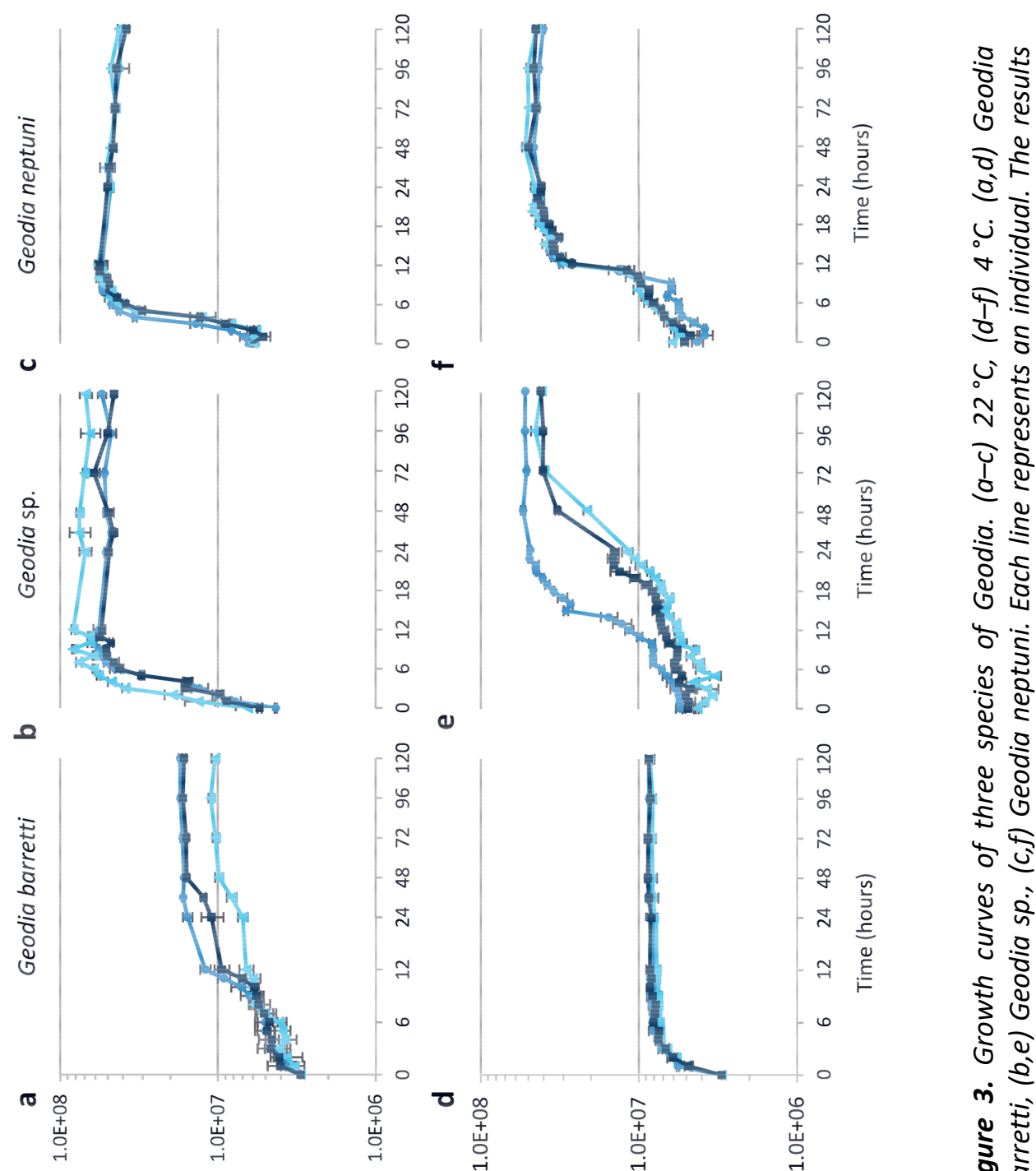

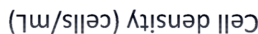

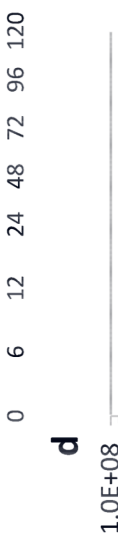

0
0
0
+
0
ப

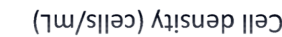

욛

co

ن

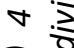

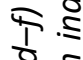

อ

Uั

$\approx$ के

过

पे के

둥

$\circ \stackrel{0}{\circ}$

: $\overline{5}$

(1) w

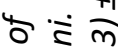

थ

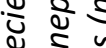

०

के 응

$\approx 0$

¿ 8

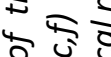

ज

$\int^{2} \frac{8}{4}$

S용

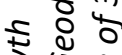

ริ ธั

जे बे

¿

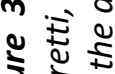

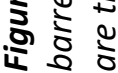

כ.22 
Table 1. Population doublings $\left(N_{d}\right)$ for growth curve and subculture/passaging experiments. The results are the average of 3 technical replicates $(n=3) \pm$ standard deviation.

\begin{tabular}{|c|c|c|c|c|c|c|c|}
\hline \multirow{2}{*}{ Experiment } & \multirow{2}{*}{$T\left({ }^{\circ} \mathrm{C}\right)$} & \multicolumn{2}{|c|}{ G. barretti } & \multicolumn{2}{|c|}{ Geodia sp. } & \multicolumn{2}{|c|}{ G. neptuni } \\
\hline & & $\mathrm{N}_{\mathrm{d}}$ & $\sigma$ & $\mathrm{N}_{\mathrm{d}}$ & $\sigma$ & $\mathrm{N}_{\mathrm{d}}$ & $\sigma$ \\
\hline \multirow{2}{*}{$\begin{array}{c}\text { Growth } \\
\text { curve }\end{array}$} & 22 & 2.26 & \pm 0.33 & 3.67 & \pm 0.12 & 3.17 & \pm 0.05 \\
\hline & 4 & 1.54 & \pm 0.18 & 3.27 & \pm 0.12 & 3.47 & \pm 0.17 \\
\hline \multirow{2}{*}{ Passaging } & 22 & 5.54 & \pm 0.17 & 4.67 & \pm 0.26 & 4.20 & \pm 0.08 \\
\hline & 4 & 6.95 & \pm 0.63 & 5.50 & \pm 0.28 & 5.53 & \pm 0.12 \\
\hline
\end{tabular}

3 individuals of $G$. neptuni incubated at $22{ }^{\circ} \mathrm{C}$ reached an average peak density of $5.54 \mathrm{E}+07$ cells $/ \mathrm{mL}$ within 12 hours (Figure $3 \mathrm{c}$ ). The average peak cell density of $\mathrm{G}$. neptuni cells incubated at $4{ }^{\circ} \mathrm{C}$ was lower (4.97E $+07 \mathrm{cells} / \mathrm{mL}$ ) (Figure $3 \mathrm{f}$ ).

\subsubsection{Cultures can be subcultured and maintained up to several weeks}

Figure 4 shows the results of subculture experiments for $G$. barretti, Geodia sp., and $\mathrm{G}$. neptuni at both $22^{\circ} \mathrm{C}$ and $4{ }^{\circ} \mathrm{C}$. Passaging times and total number of population doublings varied among the three species. As in the growth curve experiments, G. barretti cells did not reach the same density as the other two species ( $\sim 9.82 \mathrm{E}+06$ cells $/ \mathrm{mL}$ at $4{ }^{\circ} \mathrm{C}$ and $\sim 1.35 \mathrm{E}+07$ cells $/ \mathrm{mL}$ at $22^{\circ} \mathrm{C}$ ) (Figure $4 a, d)$, however, G. barretti cells continued to divide until the 5 th passage, ultimately reaching a total of 6.95 population doublings at $4^{\circ} \mathrm{C}$ and 5.54 population doublings at $22^{\circ} \mathrm{C}\left(\mathrm{N}_{\mathrm{d}}\right)$ (Table 1 ). Cell cultures of Geodia sp. had individual variations in peak cell densities when cultured at $22{ }^{\circ} \mathrm{C}$, from $3.45 \mathrm{E}+07$ to $5.77 \mathrm{E}+07$ cells $/ \mathrm{mL}$ (Figure $4 \mathrm{~b}$ ). Similarly, the peak cell density of Geodia sp. cells cultured at $4{ }^{\circ} \mathrm{C}$ varied between individuals, from $3.79 \mathrm{E}+07$ to $5.13 \mathrm{E}+07$ cells $/ \mathrm{mL}$ (Figure 4e). Geodia sp. cells cultured at $4{ }^{\circ} \mathrm{C}$ reached a higher number of population doublings $\left(N_{d}=5.50\right)$ after 28 days of culture compared to cultures at $22^{\circ} \mathrm{C}$ after 120 hours $\left(N_{d}=4.67\right.$ ) (Table 1). G. neptuni cultures reached 4.20 population doublings after 120 hours ( 5 days) (Table 1 ) but had an average peak cell density of $5.77 \mathrm{E}+07 \mathrm{cell} / \mathrm{mL}$ within 12 hours (Figure $4 \mathrm{c}$ ). On the other hand, $G$. neptuni cultured at $4{ }^{\circ} \mathrm{C}$ had 5.53 population doublings over a course of 28 days (Table 1 ) and a lower average peak cell density, only reaching $4.38 \mathrm{E}+07$ cells $/ \mathrm{mL}$ (Figure $4 \mathrm{f}$ ). 

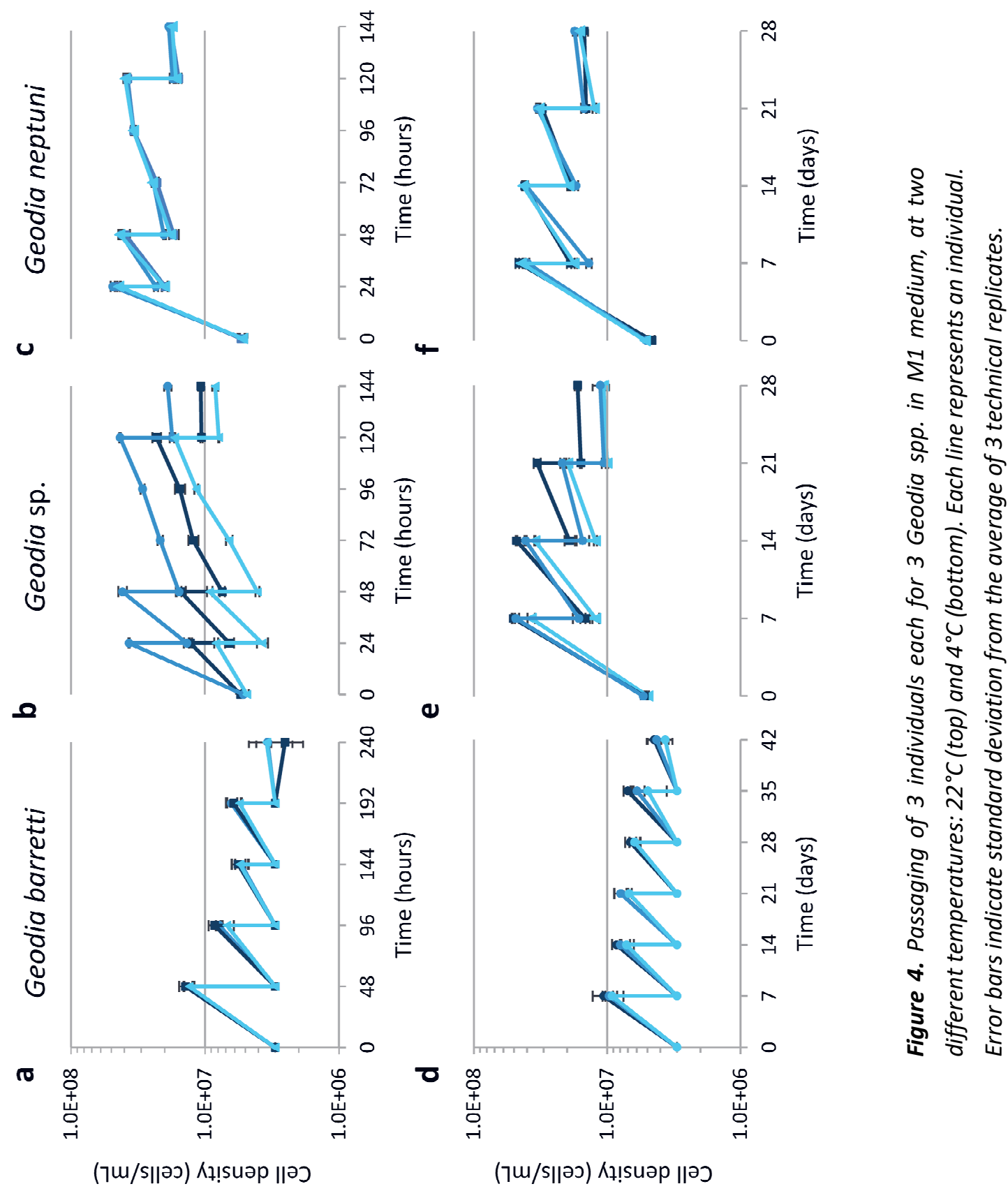

ग.22 


\subsubsection{Cell identity was verified by $18 \mathrm{~S}$ rRNA gene sequence analyses}

Cultures were routinely monitored microscopically, however, verification that the subcultures were the three Geodia species was confirmed by $18 \mathrm{~S}$ rRNA gene amplicon sequencing. For all samples sequenced, at least $99.96 \%$ of the reads had a $100 \%$ match with published sequence data for Geodia species (Table 2).

Table 2. $18 S$ rNA gene sequence match data for each species at time point zero (TO) and after one passage (P1) and four passages (P4). The results are the sequencing of one individual for each species.

\begin{tabular}{|c|c|c|c|c|c|c|}
\hline & \multicolumn{2}{|c|}{ G. barretti } & \multicolumn{2}{|c|}{ Geodia sp. } & \multicolumn{2}{|c|}{ G. neptuni } \\
\hline & TO & P4 & TO & P1 & T0 & P1 \\
\hline $\begin{array}{l}\text { Animalia, Porifera, Demospongiae, } \\
\text { Tetractinellida, Geodiidae, Geodia }\end{array}$ & 99.80 & 99.84 & 99.99 & 99.96 & 99.98 & 99.94 \\
\hline $\begin{array}{r}\text { Animalia, Porifera, Demospongiae, } \\
\text { Polymastiida, Polymastiidae }\end{array}$ & 0.20 & 0.16 & 0.00 & 0.00 & 0.00 & 0.01 \\
\hline $\begin{array}{r}\text { Animalia, Porifera, Demospongiae, } \\
\text { Haplosclerida, Niphatidae }\end{array}$ & 0.00 & 0.00 & 0.01 & 0.04 & 0.00 & 0.00 \\
\hline $\begin{array}{r}\text { Animalia, Porifera, Demospongiae, } \\
\text { Dictyoceratida, Dysideidae }\end{array}$ & 0.00 & 0.00 & 0.00 & 0.00 & 0.01 & 0.01 \\
\hline \multirow[t]{2}{*}{$\begin{array}{r}\text { Animalia, Cnidaria, Hydrozoa, } \\
\text { Anthoathecata, Hydractiniidae, } \\
\text { Clavactinia }\end{array}$} & 0.00 & 0.00 & 0.00 & 0.00 & 0.01 & 0.04 \\
\hline & $\%$ & $\%$ & $\%$ & $\%$ & $\%$ & $\%$ \\
\hline
\end{tabular}

\subsection{Discussion}

We established finite cell lines ${ }^{124,125}$ for G. barretti, Geodia sp., and G. neptuni. The cultures were monitored microscopically and were not axenic. However, sponges are holobionts with a diverse community of microbes ${ }^{126}$ that may be obligate symbionts: the microbiome of $G$. barretti is species-specific and stable $^{127}$. Continued development of sponge cell lines, and specifically, the establishment of an axenic cell line, will provide a sponge model to test hypotheses related to the functional role(s) of the sponge microbiome.As noted, the medium in cultures with dividing cells changed color, from pale orange to dark grey, and the sponge cells appeared microscopically to have dark inclusions $(\sim 0.5 \mu \mathrm{m})$. The change in medium color and appearance of dark inclusions were 
present in each individual of each species that had increases in cell number. These changes were observed as soon as cultures increased in cell number and became increasingly darker as the cell density increased. We hypothesize that the color change is associated with the production of melanin, a photoprotective pigment that has been reported from the ectosome of marine sponges ${ }^{128}$. Sponge-associated bacteria also produce melanin, causing color changes to media ${ }^{129}$. Research is in progress in our group to determine the exact cause of the color change in the medium and to characterize the inclusions present in the cells.

Both interspecific and intraspecific (individual) variation have been observed in metabolic responses of sponges ${ }^{102,103}$. Over the course of this study, inter- and intra-species responses were observed for peak cell densities, number of population doublings, passaging times and culture lifespan. Cells remained in stationary phase for up to 1 week, depending on the species and the culture temperature. Our results demonstrate the necessity of testing multiple individuals of the target species to identify the appropriate individuals for continued development of cell lines. The choice of which sponge species and even which individuals to use can have a significant impact on the outcome of the study. Selecting the appropriate species and individual source material to establish marine sponge cell lines cannot be overemphasized.

The establishment of sponge cell lines requires the optimization of several variables, including nutrient media, incubation temperature, inoculation/seeding density, duration between passages, and the use of antibiotics, to name a few. Optimization of nutrient media is especially important: A genetic algorithm approach was used to optimize the amino acid composition of M199 to improve metabolic activity in primary sponge cell cultures. M1 medium was optimized from M199, based on 48-hour cultures of one sponge species, $D$. etheria ${ }^{103}$. Even though M1 was developed for and stimulated metabolic activity in $D$. etheria, cells from this species did not divide ${ }^{103}$. Nevertheless, we hypothesized that the optimized medium would stimulate cell division in other sponge species, and our results demonstrate that M1 stimulated rapid cell division in 9 other species. M199 also stimulated cell 
division, but $M 1$ was better for most species. Since $M 1$ contains extra amino acids, for some sponges the amino acid content of M199 is suboptimal. Research is in progress to optimize other medium components (e.g., lipids, vitamins, trace metals, growth factors) (Munroe et al. in prep) and to develop cell lines from additional species of sponges. We hypothesize that, not unlike optimization of other eukaryotic cell lines, medium optimization will be required for each species and for the intended application of the cell line.

\subsection{Conclusion}

Our demonstration of exceptionally fast cell division for marine invertebrates (sponges), as well as our ability to subculture the cells, is a breakthrough in marine biotechnology. From this study, we conclude that optimization needs to be species-specific and may depend on the intended use of the cell lines. Our results form the basis for developing marine invertebrate (sponge) cell models to better understand early animal evolution and to test hypotheses related to the effects of higher temperature and lower $\mathrm{pH}$ on sponges. Furthermore, sponge cell lines may be used to scale-up production of sponge-derived chemicals with pharmaceutical relevance, and to gain more insight into the role of secondary metabolites in sponges to develop new models for marine natural products drug discovery.

\subsection{Acknowledgements}

This research was supported by the European Union Marie Curie Grant (ITN2013-BluePharmTrain-607786) (to D.S.), the European Union Horizon 2020 Project SponGES (grant agreement No. 679848) (to D.S., S.P., D.M.), the Harbor Branch Oceanographic Institute Foundation, Aquaculture and Save Our Seas Specialty License Program (to S.P.), and the National Oceanic and Atmospheric Administration, Cooperative Institute for Ocean Exploration, Research, and Technology (award number NA14OAR43202600 (to S.P.). This document reflects only the authors' views; sponsors are not responsible for any use that may be made of the information it contains. We thank Dr. M. Cristina Diaz (HBOI-FAU) and Dr. Patricia Blackwelder (Nova Southeastern University) for microscopic 
analyses and discussions regarding the color change in nutrient media. We thank Dr. Hans Tore Rapp (University of Bergen) for collecting Geodia barretti samples. S.P. also acknowledges the many collaborators, undergraduate and graduate students (especially Dr. Robin Willoughby and Dr. Klaske Schippers), postdoctoral fellows, and research assistants whose contributions to the sponge biotechnology programs at Florida Atlantic University-Harbor Branch Oceanographic Institute and Wageningen University provided the foundation for the success achieved in this study. 


\subsection{Supplementary information}

Table S1. Composition of the three media used in this studv.

\begin{tabular}{|c|c|c|c|c|}
\hline \multirow{2}{*}{\multicolumn{2}{|c|}{ Component }} & ASW & Medium 199 & M1 \\
\hline & & \multicolumn{3}{|c|}{$\mathrm{g} / \mathrm{L}$} \\
\hline \multirow{7}{*}{ Salts } & $\mathrm{NaCl}$ & 23.300 & 6.800 & 15.420 \\
\hline & Trizma HCL & 4.020 & - & 3.769 \\
\hline & Trizma Base & 2.970 & - & 2.784 \\
\hline & $\mathrm{MgCl}_{2}$ & 10.200 & - & 10.04 \\
\hline & $\mathrm{Na}_{2} \mathrm{SO}_{4}$ & 1.000 & - & 0.814 \\
\hline & $\mathrm{CaCl}_{2}$ & 1.000 & 0.200 & 0.400 \\
\hline & $\mathrm{KCl}$ & 1.000 & 0.400 & 0.302 \\
\hline \multirow{22}{*}{$\begin{array}{l}\text { Amino } \\
\text { Acids }\end{array}$} & L-Alanine & - & 0.025 & 0.053 \\
\hline & L-Arginine $\cdot \mathrm{HCL}$ & - & 0.070 & 0.095 \\
\hline & L-Aspartic Acid & - & 0.030 & 0.077 \\
\hline & L-Asparagine & - & - & 0.103 \\
\hline & L-Cystine $\cdot 2 \mathrm{HCl}$ & - & 0 & 0 \\
\hline & L-Cystine $\cdot \mathrm{HCl} \cdot \mathrm{H}_{2} \mathrm{O}$ & - & 0.026 & 0.067 \\
\hline & L-Glutamic Acid & - & 0.067 & 0.114 \\
\hline & L-Glutamine & - & 0.100 & 0.163 \\
\hline & Glycine & - & 0.050 & 0.053 \\
\hline & Hydroxy-L-Proline & - & 0.010 & 0.010 \\
\hline & L-Histidine $\cdot \mathrm{HCl} \cdot \mathrm{H}_{2} \mathrm{O}$ & - & 0.022 & 0.131 \\
\hline & L-Isoleucine & - & 0.020 & 0.070 \\
\hline & L-Leucine & - & 0.060 & 0.113 \\
\hline & L-Lysine $\cdot \mathrm{HCl}$ & - & 0.070 & 0.089 \\
\hline & L-Methionine & - & 0.015 & 0.081 \\
\hline & L-Phenylalanine & - & 0.025 & 0.963 \\
\hline & L-Proline & - & 0.040 & 0.118 \\
\hline & L-Serine & - & 0.025 & 0.869 \\
\hline & L-Threonine & - & 0.03 & 0.055 \\
\hline & L-Tryptophan & - & 0.010 & 0.729 \\
\hline & $\mathrm{L}$-Tyrosine $\cdot 2 \mathrm{Na} \cdot 2 \mathrm{H}_{2} \mathrm{O}$ & - & 0.058 & 0.105 \\
\hline & L-Valine & - & 0.025 & 0.128 \\
\hline \multirow{2}{*}{$*$} & Rifampicin & - & 0.030 & 0.030 \\
\hline & Amphotericin B & - & 0.003 & 0.003 \\
\hline
\end{tabular}

* Added antibiotics and antimycotics 
0 


\section{Chapter 3}

\section{Establishing the First Continuous Marine Sponge Cell Line}

This chapter will be submitted for publication in Nature Biotechnology as:

Hesp, K., van der Heijden, J.M.E., Munroe, S., et al. Establishing the First Continuous Marine Sponge Cell Line. Nat Biotech (2021) 


\section{Abstract}

The potential of sponge-derived chemicals for pharmaceutical applications remains largely unexploited due to insufficient biomass. Although many have attempted to culture marine sponge cells in vitro to create a scalable production platform for such biopharmaceuticals, these efforts have been mostly unsuccessful. We recently showed that Geodia barretti cells could divide rapidly in M1 medium. Here we report establishing the first marine sponge cell line, originating from G. barretti. G. barretti cells cultured in OpM1 medium, the successor of $M 1$, grew more rapidly and to a higher density than in $M 1$ medium. The maximum number of population doublings increased from 5 doublings in $\mathrm{M} 1$ to nearly 100 doublings in OpM1. Subcultured cells could be cryopreserved and used to inoculate new cultures. With these results, we have overcome the obstacle that has blocked the path to producing biopharmaceuticals with sponge cells at industrial scale for decades. 


\subsection{Introduction}

Sponges (Phylum Porifera) are one of the richest natural sources of pharmaceutically relevant chemicals ${ }^{9}, 11,94$, and contain structural components with potential human health applications in tissue engineering, for example, as a scaffold to grow bone implants ${ }^{130-134}$. The discovery of sponge-derived chemicals with pharmaceutical relevance sparked an interest in the culture of marine sponges to overcome the bottleneck of obtaining sufficient quantities of the compounds for clinical trials and commercialization ${ }^{58,60,90,111,117 . ~ M o s t ~}$ efforts to overcome this supply issue have focused on biomass production using

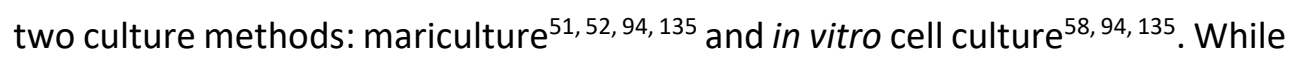
mariculture has shown promise in some cases ${ }^{51,52}$, in vitro culture of sponge cells has major advantages: it allows for close monitoring and control of culture conditions for optimization of growth and productivity and it is highly scalable. Despite the efforts of many groups, for a long time only primary cell cultures could be established. Main obstacles in sponge cell culture are a lack of starting material capable of cell division, contamination by microbial symbionts and a limited understanding of the nutritional requirements of sponge cells in vitro ${ }^{58}$.

Recently, we reported that cells of multiple sponge species were capable of rapid cell division in $\mathrm{M}^{136}$, a sponge-specific medium developed by Munroe et al. in 2019 ${ }^{103}$. M1 medium is based on the defined M199 medium ${ }^{103}$, that was previously modified and used to set up primary cultures of cells for various sponge species ${ }^{47,54,56,81}$. Amino acid concentrations were fine-tuned through multiple rounds of optimization using a genetic algorithm ${ }^{103}$. Cells from grass flat sponges Geodia sp. and Amphimedon erina, reef sponge Geodia neptuni and boreal deep-sea sponge Geodia barretti, among other species, proliferated in M1. Cultures of all 3 Geodia species divided rapidly until plateauing and could be passaged by subculturing up to 5 times and reach up to $6.95 \pm 0.63$ population doublings $\left(\mathrm{N}_{\mathrm{d}}\right)$ depending on the species. This was the first report of a concrete lead for developing stable or continuous marine sponge cell lines ${ }^{136}$.

While these results represented a breakthrough, the number of passages and population doublings that can be reached in M1 medium do not yield enough 
biomass for an industrial scale production process. Growth characteristics and longevity of sponge cells in vitro need to be improved for biomass production at such a scale to be feasible. To this end, Munroe et al. used M1 medium as a base to optimize other components such as vitamins, fetal bovine serum and several growth factors (Munroe et al., in prep) using the same genetic algorithm that was used to develop M1 medium ${ }^{103}$. This resulted in the development of a new medium composition, OpM1 (Munroe et al., in prep). We hypothesized that $G$. barretti cells cultured in OpM1 medium would have improved growth characteristics, such as a higher growth rate, maximum cell density and maximum number of population doublings, due to the additional nutrients and other components. In this study we compared these growth characteristics of $G$. barretti cells in $\mathrm{M} 1$ and $\mathrm{OpM} 1$ media.

\subsection{Materials \& Methods}

\subsubsection{Sample collection, dissociation \& cryopreservation}

Three individuals of Geodia barretti (Phylum Porifera, Class Demospongiae, Order Tetractinellida, Family Geodiidae) were collected from the ocean floor in a single trawl at a depth of $\sim 500$ meters in a fjord close to Bergen, Norway $\left(59^{\circ} 58.8^{\prime \prime} \mathrm{N} 5^{\circ} 22.4^{\prime \prime} \mathrm{E}\right)$. Cells were dissociated by squeezing fragments of the sponge through sterile gauze (B. Braun Medical, grade 16 mesh), and the resulting cell suspension was passed through a $40 \mu \mathrm{m}$ filter to remove debris. Two wash steps followed, each consisting of centrifugation at $300 \times g$ for 5 minutes and resuspension of the pellet in ASW. Cells were counted microscopically using hemocytometers (C-Chip ${ }^{\mathrm{TM}}$ Neubauer Improved, NanoEnTek) to determine cell concentrations. The cells were centrifuged once more at $300 \times g$ for 5 minutes and resuspended in cryoprotectant (10\% fetal bovine serum (FBS) and $10 \%$ dimethyl sulfoxide (DMSO) in ASW) at a density of approximately $1.0 \mathrm{E}+08$ cells $/ \mathrm{mL}$. Aliquots of $1 \mathrm{~mL}$ cell suspension in cryogenic vials were placed inside Nalgene ${ }^{\circ} \mathrm{Mr}$. Frosty freezing containers, that cooled the cell suspension at a steady rate of $1^{\circ} \mathrm{C} /$ minute when placed at $-80^{\circ} \mathrm{C}$. 


\subsubsection{Medium preparation}

$\mathrm{M} 1^{103}$ and OpM1 (Munroe et al., in prep) culture media compositions were as described in the original formulae. Stock and final concentrations, manufacturer and catalog number for each component can be found in Table 1. Salt solutions added to $\mathrm{M} 199$ powder in $\mathrm{dH}_{2} \mathrm{O}$ resulted in a final osmolality and composition close to sea water ( $\approx 1000 \mathrm{mOsm})$. Next, stock solutions for each amino acid were added to reach the desired final concentration. Before the final addition of $\mathrm{dH}_{2} \mathrm{O}$, antimicrobials ( $30 \mu \mathrm{g} / \mathrm{mL}$ rifampicin and $3 \mu \mathrm{g} / \mathrm{mL}$ amphotericin $\mathrm{B}$ ) and additional OpM1 ingredients, aliquots of the medium were stored at $-20^{\circ} \mathrm{C}$ for up to 1 month. When medium was required, a thawed aliquot could either be supplemented with $\mathrm{dH}_{2} \mathrm{O}$ and antimicrobials to make $\mathrm{M} 1$ medium, or with $\mathrm{dH}_{2} \mathrm{O}$, antimicrobials and the other ingredients required to make OpM1. The final $\mathrm{pH}$ of both media was $\approx 8.2$, similar to the $\mathrm{pH}$ of sea water. The concentrations of components in $\mathrm{M} 1$ and $\mathrm{OpM} 1$ during medium preparation were adjusted to account for dilution by addition of the inoculum, $\mathrm{dH}_{2} \mathrm{O}$ and antimicrobials. The inoculum always made up $1 / 16$ of the total culture volume and contained $16 \mathrm{x}$ the inoculation density of $3.0 \mathrm{E}+06$ cells $/ \mathrm{mL}: 4.8 \mathrm{E}+07$ cells $/ \mathrm{mL}$ in ASW.

\subsubsection{Growth characterization}

To characterize the growth of $G$. barretti cells in M1 and OpM1 media, cells from three individuals of $G$. barretti were cultured at $4^{\circ} \mathrm{C}$ for 2 days $(t=48 \mathrm{~h}$ ). Cryopreserved cells of each individual were thawed rapidly in a water bath set to $50{ }^{\circ} \mathrm{C}$, then transferred to $1.5 \mathrm{~mL}$ Eppendorf tubes. The cells were washed twice in ASW by centrifugation at $300 \times g$ for 5 minutes, removal of the supernatant and subsequent resuspension in $1 \mathrm{~mL}$ ASW. Cell concentrations were obtained using disposable hemocytometers (C-Chip ${ }^{\mathrm{TM}}$ Neubauer Improved, NanoEnTek). The cell suspension used to inoculate the culture was $1 / 16$ of the final culture volume and always contained $4.8 \mathrm{E}+07$ cells $/ \mathrm{mL}$ in ASW, to obtain a starting cell density of $3.0 \mathrm{E}+06$ cells $/ \mathrm{mL}$. The dilution of medium components by addition of the inoculum was taken into account during medium preparation, so that the final concentration of each component was as described in Table 1 after addition of the inoculum. The cells were microscopically counted every hour for 
the first 6 hours, then after 24 and 48 hours. From one constantly-mixed cell suspension, triplicates of $250 \mu \mathrm{L}$ cultures $(\approx 3.0+\mathrm{E} 06 \mathrm{cells} / \mathrm{mL})$ in 48 -well plates (CELLSTAR $^{\circledR}$, Greiner Bio-one, Cat. No. 677180) were prepared for each time point (8 time points per individual).

\subsubsection{Subculturing G. barretti cells}

Cells of three $G$. barretti individuals were cultured at $4^{\circ} \mathrm{C}$ in triplicate in 48 -well plates (CELLSTAR ${ }^{\oplus}$, Greiner Bio-one, Cat. No. 677180), with $250 \mu \mathrm{L}$ cell suspension per well and a seeding density of 3.0+E06 cells $/ \mathrm{mL}$. Cultures were passaged every 3 or 4 days. Cells that adhered to the bottom of the well were resuspended in the spent medium by pipetting up and down, then cell concentrations were determined microscopically, as described above. Subsequently, part of the cell suspension was mixed with fresh M1 or OpM1 medium to dilute back to the starting density in a total volume of $250 \mu \mathrm{L}$ per well. This was continued until the cells were no longer dividing to determine the maximum number of population doublings of the cultures in each medium.

\subsubsection{G. barretti cell bank}

Leftover cell suspension from subculturing in OpM1 was cryopreserved to create a cell bank for each cell line. The method described in the section 'Sample collection, dissociation \& cryopreservation' was used, except that OpM1 was used instead of ASW in the cryoprotectant (with 10\% FBS and 10\% DMSO) and the cell density was lower, around $1.0 \mathrm{E}+07$ cells $/ \mathrm{mL}$. To start a new culture from the cryopreserved cells in the cell bank, cells of $1 \mathrm{G}$. barretti individual were thawed and washed twice with ASW, following the protocol described in 'Growth characterization'. Cells were then inoculated in triplicate in 48-well plates (CELLSTAR ${ }^{\circledR}$, Greiner Bio-one, Cat. No. 677180), with $250 \mu \mathrm{L}$ cell suspension per well and a seeding density of 3.0+E06 cells $/ \mathrm{mL}$, and subcultured following the method described above. 


\subsubsection{Additional OpM1 components varyingly impact cell density}

OpM1 contains 9 additional components compared to M1: growth factor cocktail 1 (GF1), Platelet-derived growth factor (PDGF), pifithrin- $\alpha$ (PFT $\alpha$ ), fetal bovine serum (FBS), Insulin-Transferrin-Selenium-Ethanolamine (ITSX), Lipid Mixture-1 (LM1), vitamin solution 1 (Vit1), trace elements solution (TE), and RPMI 1640 vitamin solution (RPMI). Effects of each component were assessed by comparing growth of cells from 1 individual of G. barretti in a set of media, in each of which a different component was absent, using OpM1 and M1 media as controls. While the cell density was lower in all-but-one medium missing a component, the impact of different components on the maximum cell density varied (Figure S1).

Without GF1, which consists of epidermal growth factor (EGF), insulin-like growth factor-1 (IGF-1) and phytohemagglutinin (PHA) (Table 1), the growth curve was the same as in M1 medium. When PHA, IGF-1 and EGF were tested separately, all medium compositions without PHA performed like M1, while the composition with PHA showed no difference with the OpM1 control (Figure S2A). The effect of PHA on cell density was dose-dependent: concentrations below $75 \%$ of the PHA concentration in OpM1 decreased the final density of $G$. barretti cells (Figure S3A). Concentrations of PHA above $100 \%$ (up to $800 \%$ ) did not increase the final cell density compared to OpM1 (Figure S3B).

Absence of small chemical apoptosis inhibitor PFTa or FBS lowered the cell density compared to the OpM1 control after 6 hours, and after 24 hours the density had decreased to the same level as in the M1 control (Figure S1A,B). When Vit1 (Table 1) was omitted, the final density significantly decreased compared to OpM1 (Figure S1C). Cells cultured in OpM1 without Vit1 but with either of its components, sodium ascorbate (SA) and sodium pyruvate (SP), reached a lower density than in OpM1 after 6 hours, but after 24 hours the difference with OpM1 was no longer significant (Figure S2B). In OpM1 without PDGF, LM1, ITSX or RPMI, a modest but significant decrease in cell density was observed (Figure S1A-C). TE (Table 1) was the only component of which omission did not significantly affect the final cell density (Figure S1C). 


\subsection{Results}

\subsubsection{OpM1 medium increases maximum cell density of G. barretti in culture}

To compare characteristics of sponge cell proliferation in M1 and OpM1, growth curves of cells from 3 individuals of $G$. barretti cultured at $4{ }^{\circ} \mathrm{C}$ in both media were measured (Figure 1). Cells of $3 \mathrm{G}$. barretti individuals cultured in M1 reached an $\mathrm{N}_{d}$ of $0.7 \pm 0.1$ after 30 minutes ( $\mathrm{t}=0.5 \mathrm{~h}$ ), while cells cultured in OpM1 reached an $\mathrm{N}_{d}$ of $1.7 \pm 0.1$ in the same amount of time (Figure 1). Cells in $\mathrm{M} 1$ peaked at a cell density of $6.4 \mathrm{E}+06 \pm 5.4 \mathrm{E}+05$ cells $/ \mathrm{mL}$ on average within 5 hours after inoculation (Figure 1). These results are consistent with results we reported previously, where cells of 3 other $G$. barretti individuals cultured in M1 at $4^{\circ} \mathrm{C}$ reached an average maximum cell density of $8.5 \mathrm{E}+06 \pm 7.0 \mathrm{E}+04$ cells $/ \mathrm{mL}^{136}$. In OpM1 medium, cells of $3 \mathrm{G}$. barretti individuals plateaued at a significantly ( $p=1.6 \mathrm{E}-10$ ) higher average density of $1.2 \mathrm{E}+07 \pm 2.3 \mathrm{E}+05$ cells $/ \mathrm{mL}$ after 2 hours (Figure 1). After reaching their respective plateaus, the cells remained at the same density until the end of the culture $(t=48 \mathrm{~h})$ in both media, with the final cell densities averaging $6.9 \mathrm{E}+06 \pm 1.2 \mathrm{E}+05$ in $\mathrm{M} 1$ and significantly higher in OpM1 1.3E+07 $\pm 3.0 \mathrm{E}+05$ cells $/ \mathrm{mL}(\mathrm{p}=5.7 \mathrm{E}-14)$ (Figure 1).

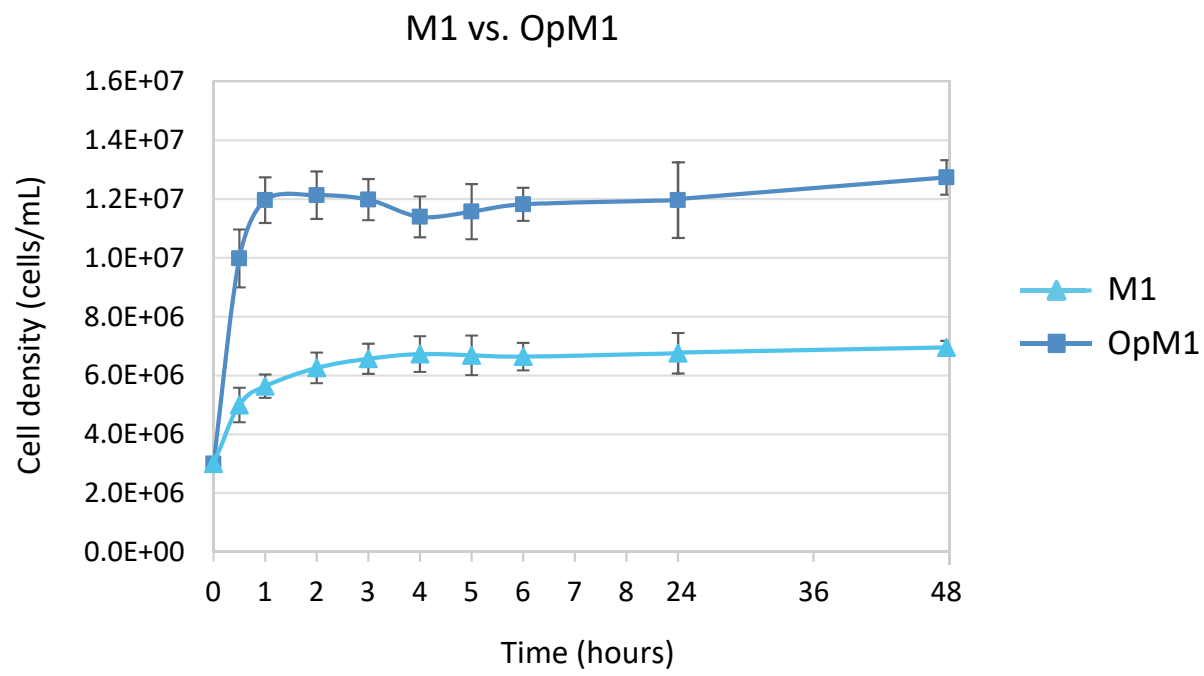

Figure 1. Growth curves of G. barretti cells cultured in M1 and OpM1 media. Error bars indicate the standard deviation from the average of biological $(N=3)$ and technical $(n=3)$ replicates. 


\subsubsection{G. barretti cells can double nearly 100 times in OpM1 medium}

Subculture experiments were carried out in both $\mathrm{M} 1$ and $\mathrm{OpM} 1$ media at $4^{\circ} \mathrm{C}$ to determine the maximum number of population doublings $\left(\mathrm{N}_{\mathrm{dmax}}\right)$ and number of passages that can be reached by cells from $3 \mathrm{G}$. barretti individuals. In M1 medium, cells that were subcultured 2 times a week continued to divide until the $5^{\text {th }}$ passage (P5) (Figure 2). In the first 2 passages (P1, P2), significant variation was observed in the cell densities reached among the $3 \mathrm{G}$. barretti individuals that were cultured in $\mathrm{M} 1$. In P1, the cells of 1 individual (GB1) reached $5.9 \mathrm{E}+06$ $\pm 3.6 \mathrm{E}+05$ cells $/ \mathrm{mL}$, while the other 2 individuals reached higher cell densities of $1.0 \mathrm{E}+07 \pm 6.6 \mathrm{E}+05(\mathrm{~GB} 2)$ and $8.1 \mathrm{E}+06 \pm 5.1 \mathrm{E}+05$ cells $/ \mathrm{mL}(\mathrm{GB} 3)$, respectively (Figure 2). A similar pattern was observed in $\mathrm{P} 2$, where these individuals reached cell densities of $6.4 \mathrm{E}+06 \pm 2.9 \mathrm{E}+05(\mathrm{~GB} 1), 9.8 \mathrm{E}+06 \pm 4.6 \mathrm{E}+05(\mathrm{~GB} 2)$, and 7.7E+06 $\pm 4.5 \mathrm{E}+05$ (GB3) cells $/ \mathrm{mL}$, respectively (Figure 2). Although this pattern of individual variation was not observed in the cell densities measured for the $3^{\text {rd }}$ (P3) and $4^{\text {th }}(\mathrm{P} 4)$ passage, it returned in P5. The maximum cell density for each individual was inconsistent between experiments, as GB2 and GB3 did not reach higher cell densities than GB1 in the growth curve (Figure 1). Cells in OpM1 medium reached higher cell densities than those observed in $M 1$ (Figure 2). In $\mathrm{P} 1$ and $\mathrm{P} 2$, the cells from $3 \mathrm{G}$. barretti individuals reached an average cell density of $1.3 \mathrm{E}+07 \pm 8.6 \mathrm{E}+05$ and $1.3 \mathrm{E}+07 \pm 2.6 \mathrm{E}+05$ cells $/ \mathrm{mL}$, respectively. In contrast to $M 1$, little individual variation was observed when cells were cultured in OpM1 medium (Figure 2). Cells of $3 \mathrm{G}$. barretti individuals cultured in M1 medium reached an average $\mathrm{N}_{\mathrm{dmax}}=5.1 \pm 0.9$ population doublings at P5 (Figure 3).

For M1 medium the $\mathrm{N}_{d}$ per passage was the highest in the first 3 passages and the cumulative $N_{d}$ then plateaued, with only minor increases in cell number over the last 2 passages (Figure $2 \&$ Figure 3 ). In contrast, for $G$. barretti cells cultured in OpM1, the $\mathrm{N}_{d}$ per passage was nearly constant at $1.8 \pm 0.02$ over 19 passages. After P19, cell densities increased for 3 passages, then decreased and cells stopped growing after 25 passages and $N_{d}=46.9 \pm 0.7$ (Figure 2). 


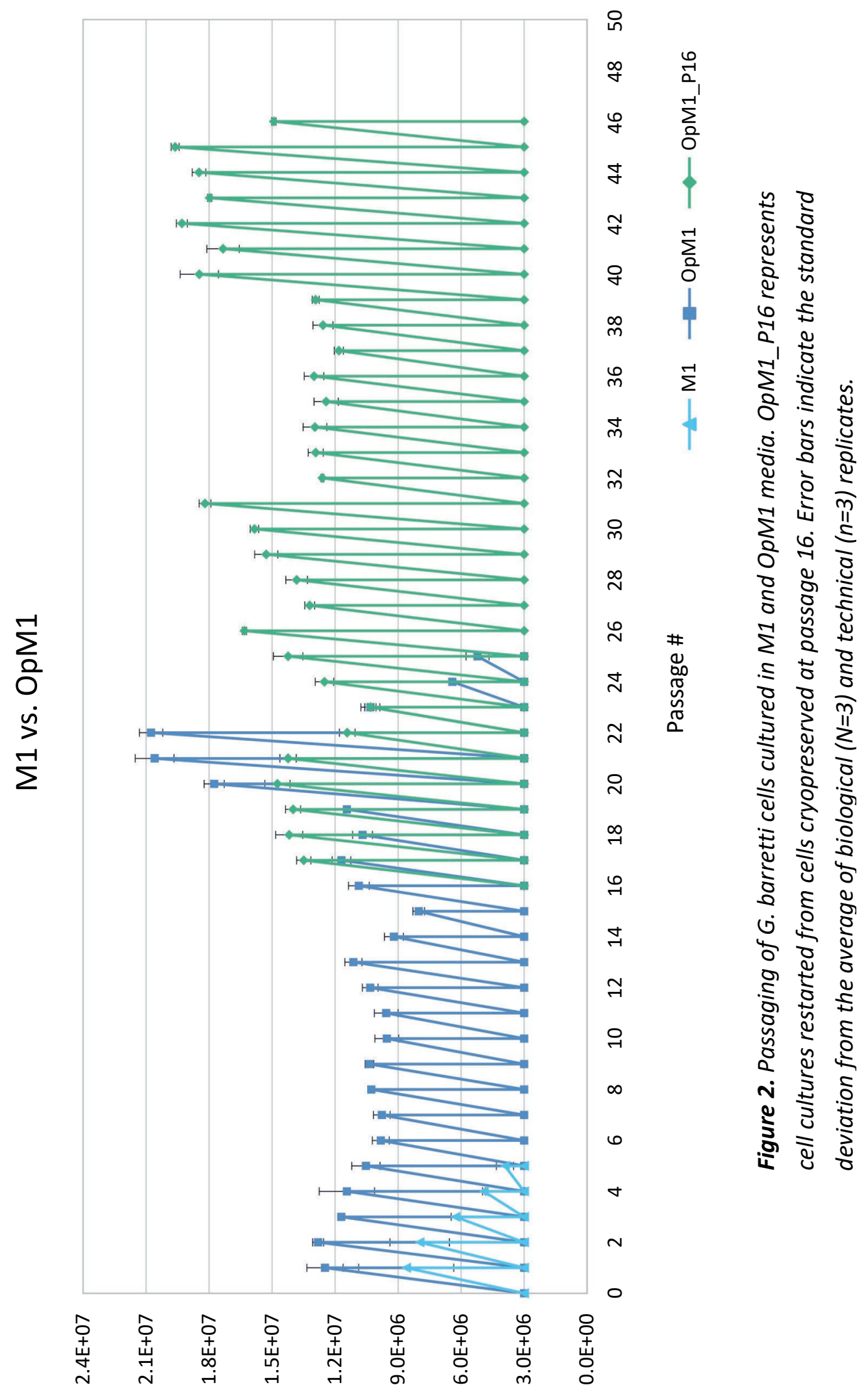


Cells that were cryopreserved at the time of the 16th passage were used to start a new culture in OpM1 medium (OpM1_P16). This new culture could be subcultured at least 30 times and cells reached a total $N_{d}=98.4 \pm 0.8$ (Figure 3). The $\mathrm{N}_{\mathrm{d}}$ reached by $G$. barretti cells cultured in OpM1 was nearly 20 times as high as the $\mathrm{N}_{\mathrm{dmax}}$ of cells cultured in M1. The cells in OpM1 continued to divide, and the $N_{\text {dmax }}$ that can be reached by $G$. barretti cells remains to be determined. Each time the cells were subcultured, a portion of the cells was cryopreserved to create a cell bank for use in future experiments.

\section{Cumulative $\mathrm{N}_{\mathrm{d}}$}

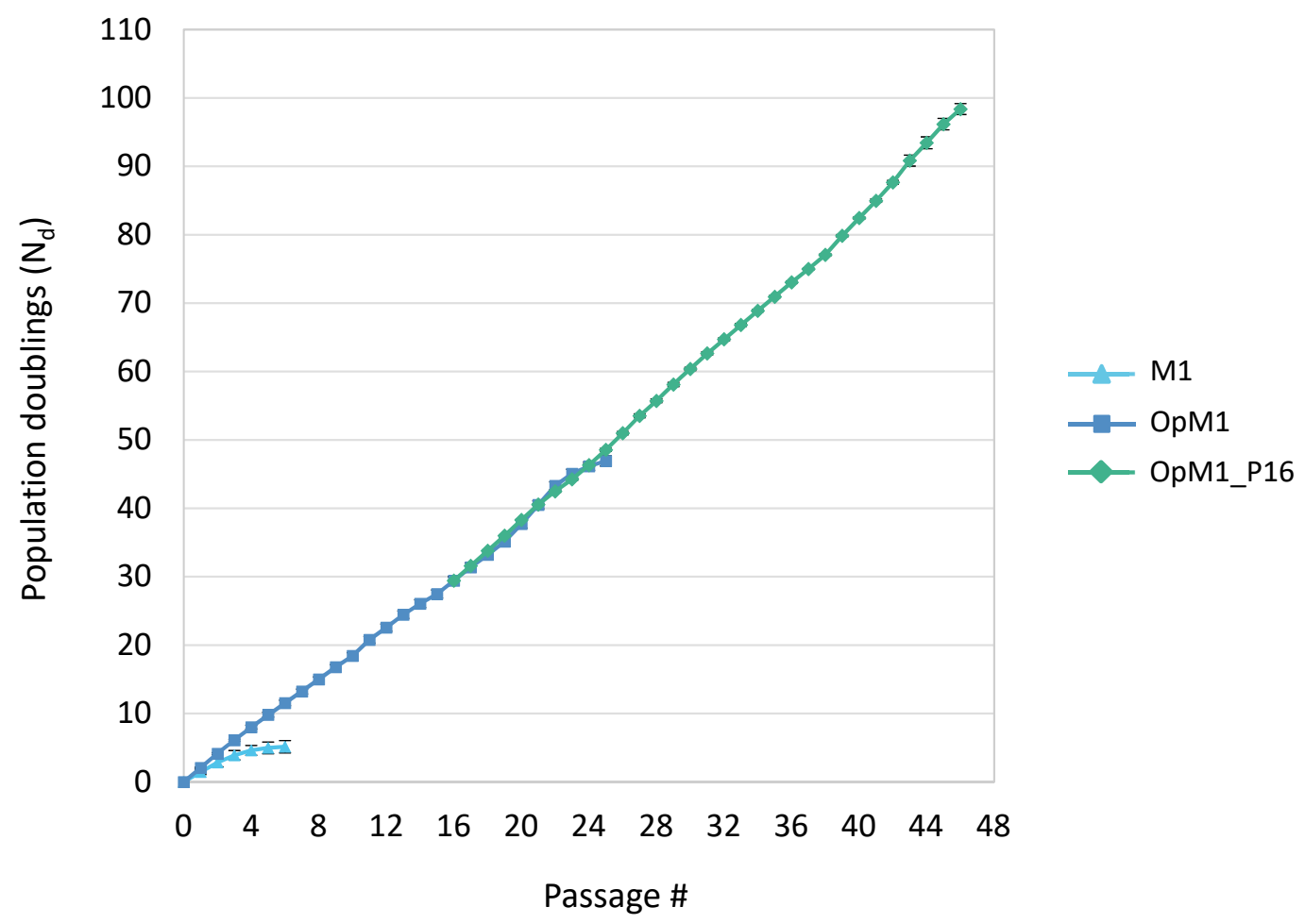

Figure 3. Cumulative population doublings ( $\left.N_{d}\right)$ of cells from 3 individuals of $G$. barretti cultured in $M 1$ and OpM1 over 5 and 25 passages, respectively. OpM1_P16 shows doublings made by cells cryopreserved after passage 16 and used to start a new culture in OpM1, which continued for at least 30 more passages (46 passages total). Error bars indicate the standard deviation from the average of biological $(\mathrm{N}=3)$ and technical $(n=3)$ replicates. 


\subsection{Discussion}

Here we report having established the first long-lasting marine sponge cell line, originating from the deep sea boreal sponge $G$. barretti. We previously reported that cells from multiple sponge species, including $G$. barretti, divided rapidly in M1 medium and could be maintained for up to 5 passages and $6.95 \pm 0.63$ population doublings ${ }^{136}$. In this study, we compared growth of $G$. barretti cells in the amino acid-optimized M1 medium ${ }^{103}$ and its successor OpM1 medium that contains optimized concentrations of additional nutrients such as growth factors, lipids, vitamins and trace elements, among others (Munroe et al., in prep). OpM1 strongly increased the growth rate and maximum cell density reached by $G$. barretti cells in culture. More importantly, the number of population doublings that could be reached by cells in OpM1 was increased nearly 20-fold, from a maximum of 5 doublings in M1 to nearly 100 doublings in OpM1. Since the cells in OpM1 continued to divide, the maximum number of population doublings that $G$. barretti cells can reach in vitro still needs to be determined. Cells that were cryopreserved after the $16^{\text {th }}$ passage resumed proliferation when thawed and used to inoculate new cultures that could be subcultured at least 30 more times, showing that the established cell line can be used to create a cell bank to store cells for future experiments.

Our results show that all additions to OpM1, except the TE solution, contributed to its superior performance compared to $\mathrm{M} 1$, but the impact of each component varied. PHA was paramount for the difference between OpM1 and M1, which is in line with previous reports of PHA-induced proliferation in cells of other marine sponges, $A$. corrugat ${ }^{54}$ and $H$. heliophila ${ }^{81}$, while removing EGF and IGF-1 did not influence the final cell density when PHA was present. Higher PHA concentrations did not increase the cell density, suggesting other factors limit the maximum cell density in G. barretti cultures. Small chemical inhibitor of p53mediated apoptosis ${ }^{137}$ PFT $\alpha$ and FBS also had a large impact, and in their absence G. barretti cell growth followed a similar pattern. After 6 hours, G. barretti cells cultured in OpM1 without either component reached higher densities than those in $M 1$, but lower than in OpM1, suggesting apoptosis occurs in the first hours. 
After 24 hours, the cell density had decreased to the same level as in M1. Therefore, we hypothesize PFT $\alpha$ and FBS can prevent apoptosis in G. barretti cells, likely via different pathways, as absence of either component results in reduced cell density. These results provide the first insights into specific nutrients required to maintain sponge cells in vitro. However, further testing is necessary to fully understand the effect of the different components in OpM1 on $G$. barretti cell growth. Additionally, how various media components affect the number of times $G$. barretti cells can divide has yet to be determined.

Strong interspecies variation has been observed in sponges ${ }^{136}$ and it remains to be determined whether OpM1 medium can support cells of species other than $G$. barretti in long-term in vitro cultures. Each species will react differently to certain medium components and culture conditions to some degree, as illustrated by differences between cells from 12 different sponge species cultured in $\mathrm{M} 1$ medium in our previous work ${ }^{136}$. Additionally, both $\mathrm{M}^{103}$ and OpM1 media (Munroe et al., in prep) were optimized for Caribbean sponge Dysidea etheria, in which it significantly increased metabolic activity but did not stimulate cells to divide. Individual variation also needs to be considered when selecting source material ${ }^{102,103,136}$, even for species with low individual variation such as $G$. barretti ${ }^{136}$. Our results show that cells from the same individual can also respond differently in separate experiments under the same culture conditions. While this could be because different medium batches and different cryovials vary slightly, it would be beneficial to repeat (sub)culture experiments when screening sponge species and individuals. Developing a production strain from any sponge species will require careful screening and selecting of source material, as well as species-specific optimizing of medium and culture conditions $^{136}$.

\subsection{Conclusion}

We established the first long-lasting marine sponge cell line, originating from deep-sea boreal sponge $G$. barretti. Studying these cells can teach us more about what sponge cells require to grow and divide and which pathways are responsible for synthesizing secondary metabolites such as barettins. In vitro 
sponge cell cultures can be used as model systems to test a wide variety of hypotheses about cell biology and how early animals evolved from unicellular organisms and developed symbiotic relationships with microbes. Our results may also function as a blueprint to establish cell lines for other sponge species. Interspecific and intraspecific (individual) variation make careful screening of source material and species- and application-specific optimizing of the culture medium essential to developing sponge cell lines. Cell lines for $G$. barretti and other sponge species can consistently produce enough biomass to develop small-scale production platforms for pharmaceutically relevant compounds. Producing biopharmaceuticals with sponge cells on an industrial scale is one step closer to becoming a reality. 


\subsection{Supplementary information}

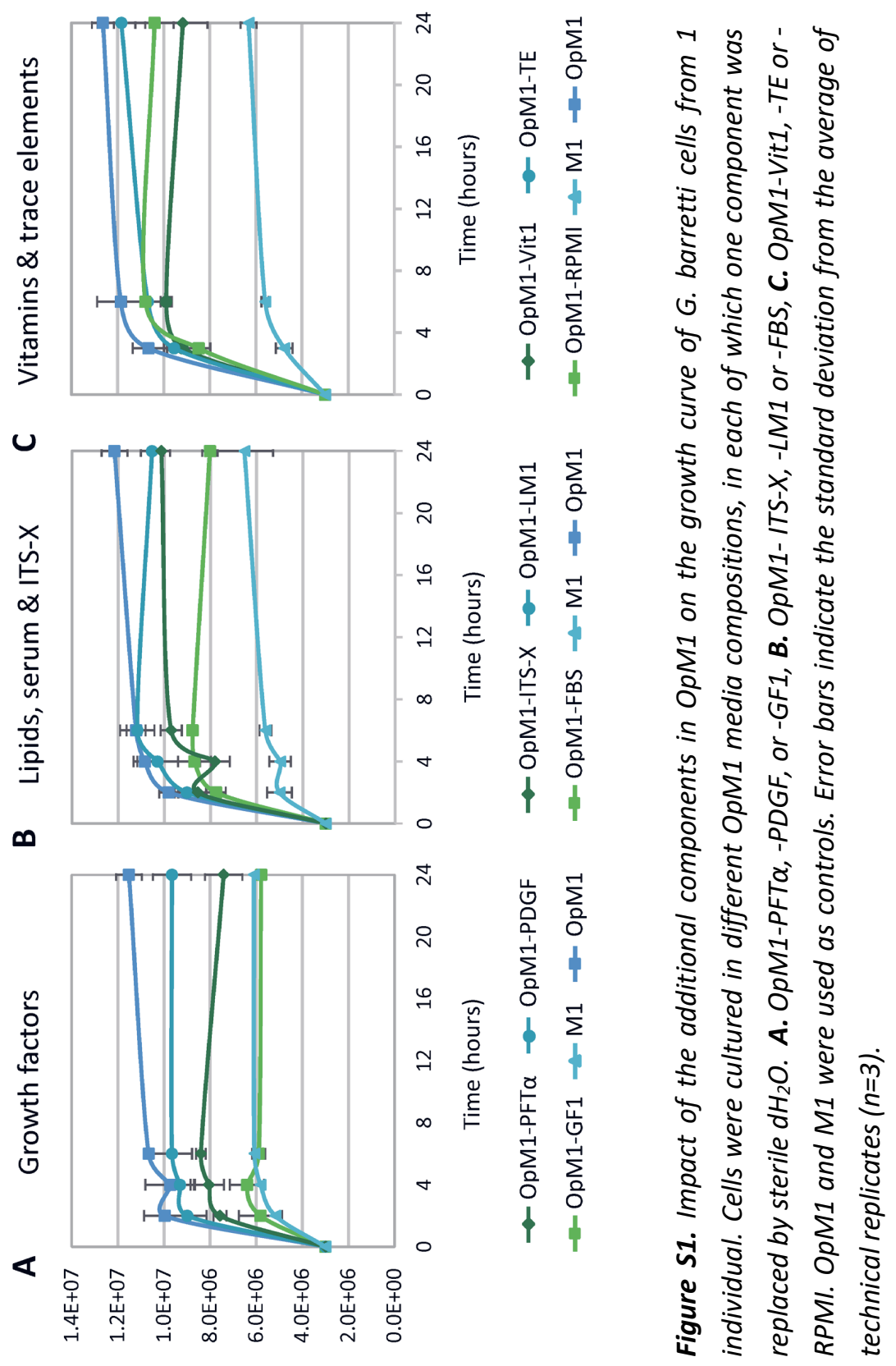

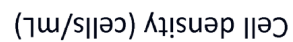




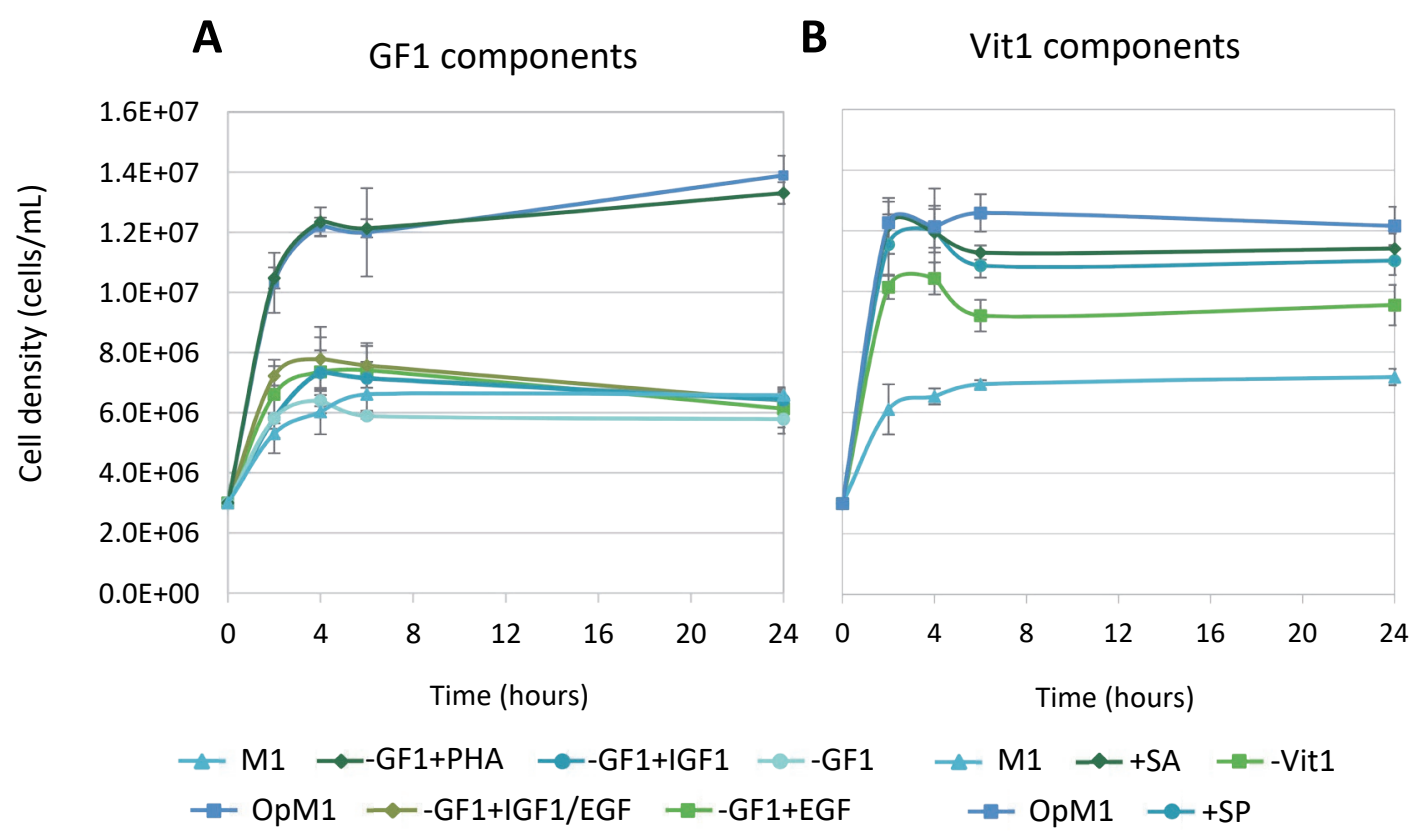

Figure S2. Growth curves of cells from $1 \mathrm{G}$. barretti individual show the impact of individual ingredients of $\boldsymbol{A}$. GF1: Cells cultured in OpM1-GF1+PHA, +IGF1, +EGF or +IGF1/EGF compared to cells cultured in OpM1-GF1, B. Vit1: Cells cultured in OpM1-Vit1, +SA or +SP compared to cells cultured in OpM1-Vit1. OpM1 and M1 were used as controls. Error bars indicate the standard deviation from the average of technical replicates $(n=3)$.

A

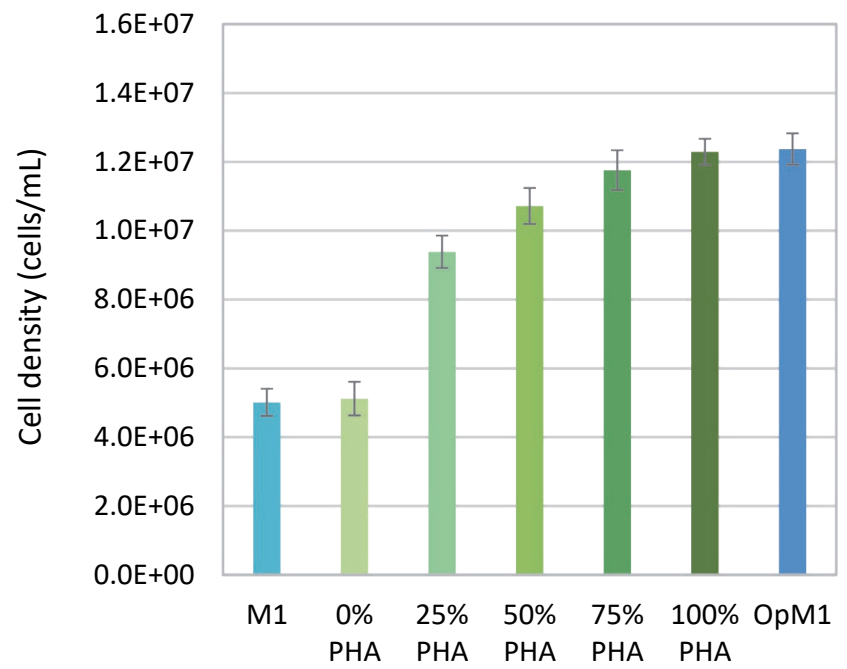

B

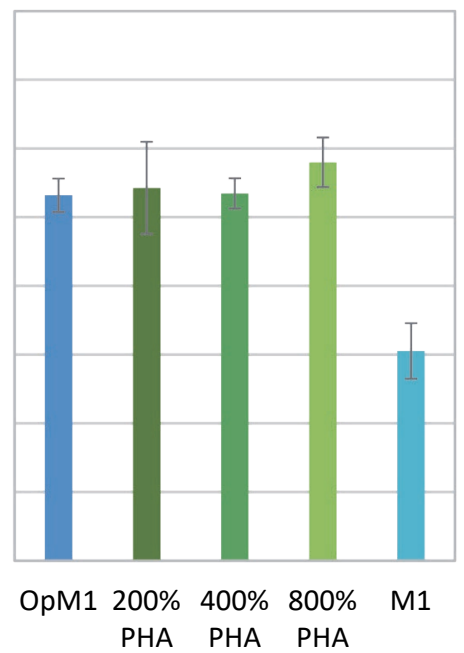

Figure S3. Dose-dependency of the final cell density reached by cells of $1 \mathrm{G}$. barretti individual on PHA concentration in OpM1 medium. OpM1-GF1 with A. 0, 25, 50, 75 and 100\%, and B. 200, 400 and $800 \%$ of the PHA concentration in OpM1. OpM1 and M1 were used as controls in both experiments. Error bars indicate the standard deviation from the average of technical replicates $(n=3)$. 
Table 1. Compositions of M1 and OpM1 media

\begin{tabular}{|c|c|c|c|c|c|}
\hline Ingredient & [x] stock & [x] M1* & [x] OpM1* & Unit & Cat. no \\
\hline M199 powder & 9.1 & 9.1 & 9.1 & $\mathrm{~g} / \mathrm{L}$ & M3769 \\
\hline $\mathrm{NaCl}$ & 275 & 15.4203 & 15.4203 & $\mathrm{~g} / \mathrm{L}$ & 488662 \\
\hline Tris-HCl & 50 & 3.7688 & 3.7688 & $\mathrm{~g} / \mathrm{L}$ & T5941 \\
\hline Tris-base & 50 & 2.7838 & 2.7838 & $\mathrm{~g} / \mathrm{L}$ & T1503 \\
\hline $\mathrm{MgCl}_{2}$ & 200 & 10.0400 & 10.0400 & $\mathrm{~g} / \mathrm{L}$ & M2393 \\
\hline $\mathrm{Na}_{2} \mathrm{SO}_{4}$ & 50 & 0.8170 & 0.8170 & $\mathrm{~g} / \mathrm{L}$ & 238597 \\
\hline $\mathrm{CaCl}_{2}$ & 50 & 0.4000 & 0.4000 & $\mathrm{~g} / \mathrm{L}$ & C7902 \\
\hline $\mathrm{KCl}$ & 50 & 0.3021 & 0.3021 & $\mathrm{~g} / \mathrm{L}$ & P5405 \\
\hline L-Alanine & 5 & 0.0281 & 0.0281 & $\mathrm{~g} / \mathrm{L}$ & A3534 \\
\hline L-Arginine & 5 & 0.0250 & 0.0250 & $\mathrm{~g} / \mathrm{L}$ & A6969 \\
\hline L-Aspartic acid & 5 & 0.0469 & 0.0469 & $\mathrm{~g} / \mathrm{L}$ & A4534 \\
\hline L-Asparagine & 5 & 0.1031 & 0.1031 & $\mathrm{~g} / \mathrm{L}$ & A0884 \\
\hline L-Cysteine & 5 & 0.0406 & 0.0406 & $\mathrm{~g} / \mathrm{L}$ & C6852 \\
\hline L-Glutamic acid & 5 & 0.0469 & 0.0469 & $\mathrm{~g} / \mathrm{L}$ & G5638 \\
\hline L-Glutamine & 5 & 0.0625 & 0.0880 & $\mathrm{~g} / \mathrm{L}$ & G8540 \\
\hline Glycine & 5 & 0.0031 & 0.0031 & $\mathrm{~g} / \mathrm{L}$ & G8790 \\
\hline L-Histidine & 5 & 0.1094 & 0.1094 & $\mathrm{~g} / \mathrm{L}$ & H8125 \\
\hline L-Isoleucine & 5 & 0.0500 & 0.0500 & $\mathrm{~g} / \mathrm{L}$ & 12752 \\
\hline L-Leucine & 5 & 0.0531 & 0.0531 & $\mathrm{~g} / \mathrm{L}$ & L8912 \\
\hline L-Lysine & 5 & 0.0188 & 0.0188 & $\mathrm{~g} / \mathrm{L}$ & L5626 \\
\hline L-Methionine & 5 & 0.0656 & 0.0656 & $\mathrm{~g} / \mathrm{L}$ & M9625 \\
\hline L-Phenylalanine & 5 & 0.0938 & 0.0938 & $\mathrm{~g} / \mathrm{L}$ & P5482 \\
\hline L-Proline & 5 & 0.0781 & 0.0781 & $\mathrm{~g} / \mathrm{L}$ & P5607 \\
\hline L-Serine & 5 & 0.0844 & 0.0844 & $\mathrm{~g} / \mathrm{L}$ & S5511 \\
\hline L-Threonine & 5 & 0.0250 & 0.0250 & $\mathrm{~g} / \mathrm{L}$ & T8441 \\
\hline L-Tryptophan & 5 & 0.0719 & 0.0719 & $\mathrm{~g} / \mathrm{L}$ & T0254 \\
\hline L-Tyrosine & 5 & 0.0469 & 0.0469 & $\mathrm{~g} / \mathrm{L}$ & T1145 \\
\hline L-Valine & 5 & 0.1031 & 0.1031 & $\mathrm{~g} / \mathrm{L}$ & V6504 \\
\hline Rifampicin & 30 & 0.0300 & 0.0300 & $\mathrm{~g} / \mathrm{L}$ & R3501 \\
\hline Amphotericin B & 0.2500 & 0.0250 & 0.0250 & $\mathrm{~g} / \mathrm{L}$ & A2411 \\
\hline Ascorbate $\cdot \mathrm{Na}$ & 0.5943 & - & 0.0015 & $\mathrm{~g} / \mathrm{L}$ & A4034 \\
\hline Pyruvate $\cdot \mathrm{Na}$ & 0.4400 & - & 0.0011 & $\mathrm{~g} / \mathrm{L}$ & P5280 \\
\hline $\mathrm{Na}_{2} \mathrm{SiO}_{3}$ & 1.4210 & - & 0.0018 & $\mathrm{~g} / \mathrm{L}$ & 307815 \\
\hline $\mathrm{ZnSO}_{4} \cdot 7 \mathrm{H}_{2} \mathrm{O}$ & $6.46 \mathrm{E}-02$ & - & 0.0001 & $\mathrm{~g} / \mathrm{L}$ & Z0251 \\
\hline Lectin from Phaseolus vulgaris (PHA) & 4 & - & $4.72 \mathrm{E}-02$ & $\mathrm{~g} / \mathrm{L}$ & L1668 \\
\hline EGF Recombinant Human Protein & $1.60 \mathrm{E}-04$ & - & 1.89E-06 & $\mathrm{g} / \mathrm{L}$ & 10605HNAE50 \\
\hline $\begin{array}{l}\text { Insulin-like Growth Factor-1 Protein, } \\
\text { Recombinant Human }\end{array}$ & $8.00 \mathrm{E}-03$ & - & $9.44 \mathrm{E}-05$ & $\mathrm{~g} / \mathrm{L}$ & GF138 \\
\hline Pifithrin- $\alpha$ & 20 & - & $1.19 \mathrm{E}-02$ & $\mathrm{~g} / \mathrm{L}$ & P4359 \\
\hline PDGF-BB Recombinant Mouse Protein & $1.00 \mathrm{E}-01$ & - & 8.69E-05 & $\mathrm{g} / \mathrm{L}$ & PMG0044 \\
\hline RPMI 1640 Vitamin solution (100x) & - & - & 3.11 & $\mu \mathrm{L} / \mathrm{mL}$ & R7256 \\
\hline $\begin{array}{l}\text { Fetal Bovine Serum, qualified, One } \\
\text { Shot }^{\mathrm{TM}} \text {, US origin }\end{array}$ & - & - & 4.35 & $\mu \mathrm{L} / \mathrm{mL}$ & A3160501 \\
\hline $\begin{array}{l}\text { Insulin Transferrin Selenium } \\
\text { Ethanolamine }\end{array}$ & - & - & 11.18 & $\mu \mathrm{L} / \mathrm{mL}$ & 51500056 \\
\hline Lipid Mixture-1 (100x) & - & - & 11.18 & $\mu \mathrm{l} / \mathrm{mL}$ & L0288 \\
\hline
\end{tabular}

*Numbers in columns [x] M1 and [x] OpM1 do not include concentrations of nutrients present in M199 powder, only what is added to prepare both media. 
0 


\section{Chapter 4}

Transcriptome Analysis Provides Insights into How Marine Sponge Geodia barretti Transitions from In Situ to In Vitro Conditions

This chapter will be submitted for publication as:

Hesp, K., Riesgo, A., Martens, D.E., et al. Transcriptome Analysis Provides Insights into How Marine Sponge Geodia barretti Transitions from In Situ to In Vitro Conditions (2021) 


\section{Abstract}

Recent progress in establishing sponge cell lines has brought us one step closer to producing sponge-derived biopharmaceuticals on an industrial scale. However, there is still a major lack of knowledge about sponge cells, how they proliferate, respond to stress, or react to nutrients and other stimuli, among others. To bridge this gap, we investigated which genes are differentially expressed in 3 states: sponge fragments, dissociated and cryopreserved cells, and cells cultured in optimized nutrient medium. Cells retained proliferative potential in all 3 states and expressed genes needed to progress the cell cycle and prevent apoptosis. Cells in culture reorganized their cytoskeleton and downregulated genes needed to aggregate and adhere to the extracellular matrix. Cells in culture relied less on lipids and glycogen reserves to make energy, and upregulated genes required to synthesize proteins and lipids. Genes for phagocytosis were expressed at lower levels, likely replaced by diffusing or transport of soluble energy sources. Our results provide the first glimpse at the changes sponge cells need to undergo to successfully transition from in situ to in vitro conditions. 


\subsection{Introduction}

Lack of sponge cell lines impedes developing new pharmaceuticals derived from marine sponges. Sponges are among the most ancient phyla of animals, as well as one of the richest natural sources of biologically active compounds. A vast array of sponge-derived natural compounds with interesting properties such as antiviral, antimicrobial and antitumor activity have already been discovered in these animals ${ }^{94,112,113}$. Obtaining a sufficient supply of these compounds is currently the most significant obstacle for pharmaceutical or commercial application of sponge-derived natural products ${ }^{58}$. Large-scale wild harvest is neither ecologically nor economically sustainable, and chemical synthesis is not feasible for many compounds due to their chemical complexity ${ }^{9}$. While mariculture has been used with some success ${ }^{51,52}$, in vitro cell culture has many characteristics that make it more attractive for production of pharmaceuticals, as it is easily scalable and allows for close monitoring and optimization of conditions during production. Until recently however, no long-lasting sponge cell cultures had been reported.

Although methods to establish primary cell cultures were developed decades ago, cells did not typically proliferate in such cultures; instead a slow shift of cells towards apoptosis occurred ${ }^{54,81}$. Sponge cells are usually obtained by harvesting whole sponges, cutting them into small fragments, and mechanically or chemically dissociating the cells ${ }^{47}, 53,54,81$. Once dissociated, cells are often cryopreserved $^{54,81,102}$ before being thawed and used to inoculate cultures in filtered sea water (FSW) or various commercially available nutrient media (reviewed by Pomponi, 2006). Cell dissociation was shown to correlate strongly with cells entering programmed cell death ${ }^{57}$, suggesting that the cells did not recover after the initial stress response. Absence of essential nutrients and contamination by symbionts have been identified as possible causes for this inability to recover ${ }^{58}$, but the exact reasons have remained largely unknown.

Two recently developed sponge-specific nutrient media, $\mathrm{M}^{103}$ and OpM1 (Munroe et al. in prep), have led to major breakthroughs in sponge cell culture. We reported that dissociated and cryopreserved cells of multiple sponge 
species, including the deep sea boreal sponge G. barretti, can be cultured and maintained over multiple passages ${ }^{136}$ in $M 1$ medium, with optimized amino acid concentrations ${ }^{103}$. Concentrations of other components including lipids, vitamins, trace elements and growth factors were optimized using $\mathrm{M} 1$ as a base, resulting in OpM1 medium (Munroe et al. in prep). G. barretti cells cultured in OpM1 underwent nearly 100 population doublings, 20 times more than the 5 doublings reached by cells cultured in $M 1$ medium, resulting in the first continuous marine sponge cell line (Hesp et al., in prep). Investigating what genes are up- or downregulated while proliferating in vitro can guide what cells require to be cultured successfully and how to achieve this in cells of sponge species that do not respond to OpM1 medium. Furthermore, investigating metabolic pathways can determine how secondary metabolites are synthesized and ultimately, how to produce such metabolites on a large scale. In this study, we compared how genes are differentially expressed between $G$. barretti sponge fragments, dissociated and cryopreserved cells, and cells cultured in OpM1 medium, to study the changes sponge cells undergo between these 3 states.

\subsection{Materials \& Methods}

\subsubsection{Sample collection}

We collected 3 individuals of Geodia barretti (Order Tetractinellida, Family Geodiidae) in a single trawl at a depth of $\approx 500$ meters $\left(59^{\circ} 58.8^{\prime \prime} \mathrm{N} 5^{\circ} 22.4^{\prime \prime} \mathrm{E}\right)$ in a fjord close to Bergen, Norway. Pieces of each individual were cut into fragments of less than $0.5 \mathrm{~cm}^{3}$, which were placed in $5-10$ volumes of RNALater, left to soak overnight at $4^{\circ} \mathrm{C}$ and then stored at $-80^{\circ} \mathrm{C}$ for RNA extraction.

\subsubsection{Cell dissociation \& cryopreservation}

From the remaining part of each individual sponge, cells were dissociated by squeezing them through a sterile gauze, then pipetted through a $40 \mu \mathrm{m}$ filter to remove debris. Cells were then centrifuged at $300 \times \mathrm{g}$ for 5 minutes, resuspended in filtered sea water (FSW) and counted manually using a hemocytometer (CChip Neubauer improved, NanoEnTek). Finally, the cells were spun down once more and resuspended in cryoprotectant ( $10 \%$ fetal bovine serum and $10 \%$ 
dimethyl sulfoxide in FSW) to achieve a concentration of $\approx 1.0+$ E08 cells $/ \mathrm{ml}$. Aliquots of $1 \mathrm{ml}$ in cryogenic vials were put inside a Nalgene ${ }^{\circledR} \mathrm{Mr}$. Frosty freezing container and placed at $-80^{\circ} \mathrm{C}$ to cool at the steady rate of $1^{\circ} \mathrm{C} / \mathrm{min}$.

\subsubsection{G. barretti cell culture \& sample preparation}

The original protocol was followed to prepare OpM1 medium (Munroe et al., in prep, Hesp et al., in prep). Rifampicin $(30 \mu \mathrm{g} / \mathrm{mL})$ and amphotericin $\mathrm{B}(3 \mu \mathrm{g} / \mathrm{mL})$ were added to prevent bacterial and fungal contamination, respectively. Geodia barretti cells were thawed rapidly in a $50{ }^{\circ} \mathrm{C}$ water bath and transferred to a sterile $1.5 \mathrm{~mL}$ Eppendorf tube, then washed twice by centrifuging at 1,500 $\mathrm{x} g$ for 5 minutes and resuspending in $1 \mathrm{~mL}$ of artificial sea water (ASW). Cells were counted manually using a hemocytometer. For each individual, a total of $\approx 1.0+$ E08 dissociated cells was placed in a $1.5 \mathrm{~mL}$ sterile Eppendorf tube and centrifuged at 1,500 $\times g$ for 5 minutes and RNA was extracted from the pellet. From the remaining cell suspension, cultures were inoculated at 3.0+E06 cells $/ \mathrm{mL}$ in $10 \mathrm{~mL} \mathrm{OpM} 1$ for each individual and incubated at $4{ }^{\circ} \mathrm{C}$. After 1 hour, the cells had more than doubled ( $\approx 1.0+E 07$ cells $/ \mathrm{mL}$ ) (Hesp et al., in prep), were centrifuged at $1,500 \times g$ for 5 minutes and RNA was extracted from the pellet.

\subsubsection{RNA extraction}

Total RNA was extracted from $100 \mathrm{mg}$ of sponge fragments, a pellet containing $\approx 1.0+$ E08 of dissociated cells, and a pellet containing $\approx 1.0+$ E08 of cultured cells for each of the 3 individuals. The Maxwell ${ }^{\circledR}$ RSC simplyRNA Cells Kit, following the manufacturer's protocol, except for the step to homogenize the sample; sponge fragments were vortexed for 30 seconds in $200 \mu \mathrm{L}$ homogenization buffer with thiolglycerol $(20 \mu \mathrm{L} / \mathrm{mL})$, while cell pellets were merely resuspended in the $200 \mu \mathrm{L}$ homogenization buffer with thiolglycerol $(20 \mu \mathrm{L} / \mathrm{mL})$ to prevent shearing. RNA concentrations as well as $\mathrm{OD}_{260} / \mathrm{OD}_{280}$ were obtained using a NanoDrop 2000 spectrophotometer (Thermo Scientific). The quality of the total RNA samples was determined using 2 different methods: agarose gel electrophoresis, to detect possible RNA degradation and contamination, and an Agilent Bioanalyzer 2100 System (Agilent Technology) to determine RNA integrity. 


\subsubsection{RNA sequencing \& de novo transcriptome assembly}

Stranded cDNA libraries derived from sponge tissues and cells were prepared from poly-A enriched mRNA using the Illumina TruSeq ${ }^{\text {TM }}$ RNA Sample Prep Kit protocol. Libraries were sequenced on the Illumina HiSeq 2000 platform with 150 bp paired-end reads (Novogene, Hong Kong) using TruSeq ${ }^{\mathrm{TM}}$ RNA Sample Prep Kit adapter sequences: RNA 5' Adapter (RA5, \#15013205) (5'AATGATACGGCGACCACCGAGATCTACACTCTTTCCCTACACGACGCTCTTCCGATCT3') and RNA 3' Adapter (RA3, \#15013207) (5'-GATCGGAAGAGCACACGTCTGAACTCCAGTCAC (6-nucleotide index) ATCTCGTATGCCGTCTTCTGCTTG-3').

De novo transcriptome assembly of reads from tissue and cell samples of all individuals $(n=9)$ combined was performed using Trinity ( $\mathrm{r} 20140413 p 1)^{138}$. All read pairs used for assembly were mapped back to the assembled transcripts using Bowtie2 (bowtie2-align-s v2.3.4exit) ${ }^{139}$ to evaluate the quality of the transcriptome assembly. Read quality was checked using Illumina CASAVA $\mathrm{v} 1.8^{140}$. Raw sequence reads were filtered to remove adapter sequences and low-quality reads to make sure downstream analyses were based on clean reads. Read pairs were discarded when either read contained adapter contamination, uncertain nucleotides constituted over 10 percent of either read, or low-quality nucleotides (base quality $<20$ ) constituted $>50 \%$ of either read.

\subsubsection{Annotation \& quantification}

Transcripts and peptide sequences for the longest open reading frames (ORFs) were compared to 7 databases: NR (e-value 1E-05), Swiss-Prot (e-value 1E-05) and KOG/COG (e-value 1E-03) using Diamond v0.8.22 $2^{141}$, KEGG (e-value 1E-08, KAAS r140224 ${ }^{142}$ ), and NT (e-value 1E-05, Blast v2.2.28+ ${ }^{143}$ ), Pfam (e-value 0.01, Hmmscan HMMER 3.1b1 ${ }^{144}$ ) and GO (e-value 1E-06, Blast2go b2g4pipe_v2.5 ${ }^{145,}$ ${ }^{146}$ ). MEGAN6 ${ }^{147}$ was used to determine the lowest common ancestor (LCA) of each transcript annotated in the Swiss-Prot database by Diamond. Reads were mapped back to the assembled transcripts for each of the 3 sponge fragments, dissociated cells and cultured cells samples separately using Bowtie (v2-2.2.2) ${ }^{139}$, with 0 mismatches allowed. Subsequently, RSEM (v1.3.0) $)^{148}$ was used to estimate how abundant individual transcripts were in each sample. 


\subsubsection{Differential expression \& gene set enrichment analysis}

Transcript expression levels were compared between DC and F, DC and CC, and $\mathrm{CC}$ and $\mathrm{F}$ samples. Differential expression was determined using DESeq2 ( $\mathrm{p}$ $0.05, v 1.10 .1)^{149}$, followed by GO enrichment using GOSeq-1.32.0 $0^{150}$ and topGo2.23.0 $151(p<0.05)$ and KEGG enrichment using KOBAS $(p<0.05, v 3.0)^{152-154}$.

\subsection{Results}

\subsubsection{De novo transcriptome assembly \& annotation}

RNA was extracted from sponge fragments (F), dissociated cells (DC) and cultured cells (CC) of $3 \mathrm{G}$. barretti individuals. Before RNA was extracted, CC were inoculated in OpM1 medium (Munroe et al., in prep, Hesp et al., in prep) and incubated at $4^{\circ} \mathrm{C}$ for $1-1.5$ hours to ensure the cells were actively dividing. PolyA enriched mRNA was sequenced on the Illumina HiSeq 2000 platform with $150 \mathrm{bp}$ paired-end reads (Novogene, Hong Kong). After reads with low quality or adapter contamination were removed, the clean reads were used in de novo transcriptome assembly. The number of raw reads per sample was between $20,138,146-28,526,078$. Of all raw reads, $98.66 \%$ passed quality control and were used to assemble the transcriptome (Table S1). Overall, the clean reads had an average GC content of $49.90 \%$ (Table S3), which was higher than the GC content found in the coding sequences of 3 other sponges, Amphimedon queenslandica (39\%), Haliclona amboinensis (42\%) and Oscarella carmela (45\%) 155 . The total number of transcripts was 204,939 , of which 25,798 transcripts were longer than 2 kilobase pairs (kbp) (Table S2). The average transcript length was 1004 base pairs (bp) and the N50 value was 1595 bp, meaning 50\% of all bp were present in transcripts of $\geq 1595 \mathrm{bp}$.

Transcripts were compared to those in 7 databases for functional gene annotation: NCBI non-redundant protein (NR) and nucleotide sequences (NT), Protein family (Pfam), eukaryotic orthologous groups (KOG) and cluster of orthologous groups (COG) of proteins, Swiss-Prot, Kyoto Encyclopedia of Genes and Genome (KEGG), and Gene Ontology (GO). The database with the most successfully annotated transcripts was $\mathrm{Nr}$ with $54.39 \%$, followed by Swiss-Prot with $45.35 \%$. Of all transcripts, 59.74\% were annotated in at least 1 database, 
while $1.54 \%$ of transcripts could be annotated in all 7 databases (Table S4). A large proportion of transcripts $(40.80 \%)$ matched with genes in NR in a single organism: A. queenslandica, the first sponge to have its full genome sequenced ${ }^{3}$. Smaller numbers of transcripts matched with genes in the golden silk orb-weaver spider Nephila clavipes (4.00\%), humans (2.50\%), stony coral Stylophora pistillata $(2.10 \%)$ and sea anemone Exaiptasia pallida (1.9\%), while the best matches for the remaining $48.70 \%$ were with genes in a variety of other species. The transcriptome was analyzed for completeness by screening for 978 core metazoan genes (Table S3), using BUSCO (v2/v3) $)^{156}$ with the gVolante interface ${ }^{157}$. Complete orthologs were found for 912 genes (93.25\%), while partials were detected for 36 more genes, for a total of 948 genes (96.93\%). Some conserved eukaryotic genes were also annotated manually by local BLAST searches of transcripts and their open reading frame(s) as identified using the free online ExPASy Translate tool, to identify the strongest gene candidates and any potential isoforms in $G$. barretti. These transcripts and the proteins they encode can be found in 2 supplementary FASTA files.

\subsubsection{Lowest common ancestor analysis}

MEGAN6 ${ }^{147}$ was used to analyze the taxonomic origin of each gene annotated in the Swissprot database, and compare the results for all transcripts in the assembled transcriptome (Figure $1 \mathrm{~A}$ ) to those for genes with a normalized readcount (NC) of $>0$ in samples from F, CC and DC (Figure 1B-D). While $18.6 \%$ of all genes in the assembly were of prokaryotic origin ( $18.0 \%$ bacterial, $0.6 \%$ archaeal) (Figure $1 \mathrm{~A}$ ), only $\sim 11 \%$ of the genes with $N C>0$ were prokaryotic, with little to no difference between each of the 3 states (Figure 1B-D). Most notable was the difference in the number of archaeal genes, which was $0.6 \%$ and $0.5 \%$ in both the assembly and $\mathrm{F}(\mathrm{NC}>0)$ (Figure $1 \mathrm{~A}, \mathrm{~B})$ and only $0.2 \%$ in both $\mathrm{CC}$ and DC (Figure $1 C, D)$. The vast majority of genes in the assembly (77\%) as well as the 3 different states ( $85 \%$ ) were of eukaryotic origin. Around $4 \%$ of all genes were not successfully assigned to any taxon. Of the eukaryotic genes, $86.5 \%$ belonged to metazoans, $0.7 \%$ to amoebozoa and $0.7 \%$ to fungi (data not shown). The metazoan genes originated overwhelmingly from bilaterians (97.8\%), likely because bilaterian genes are the most well characterized. 


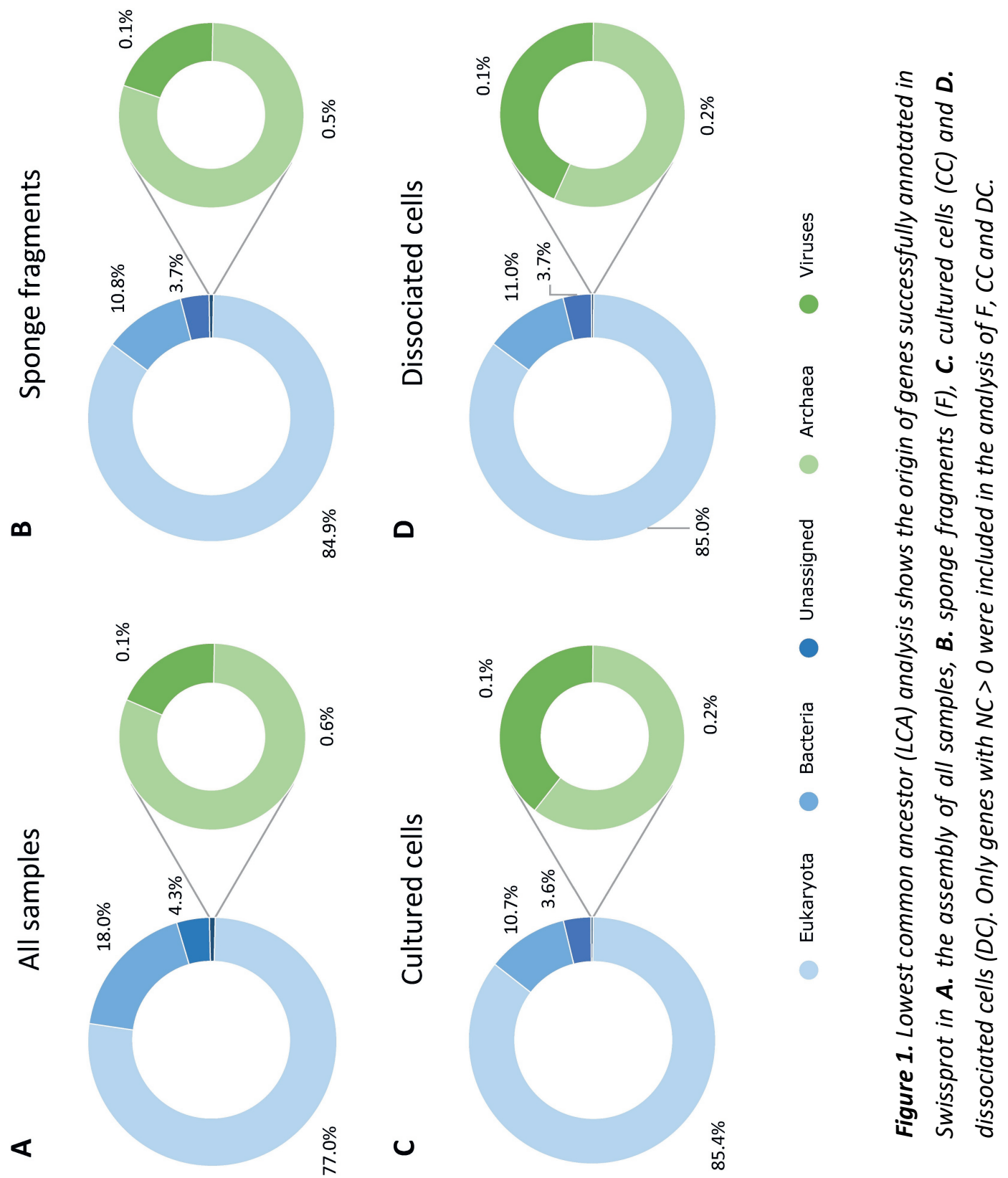



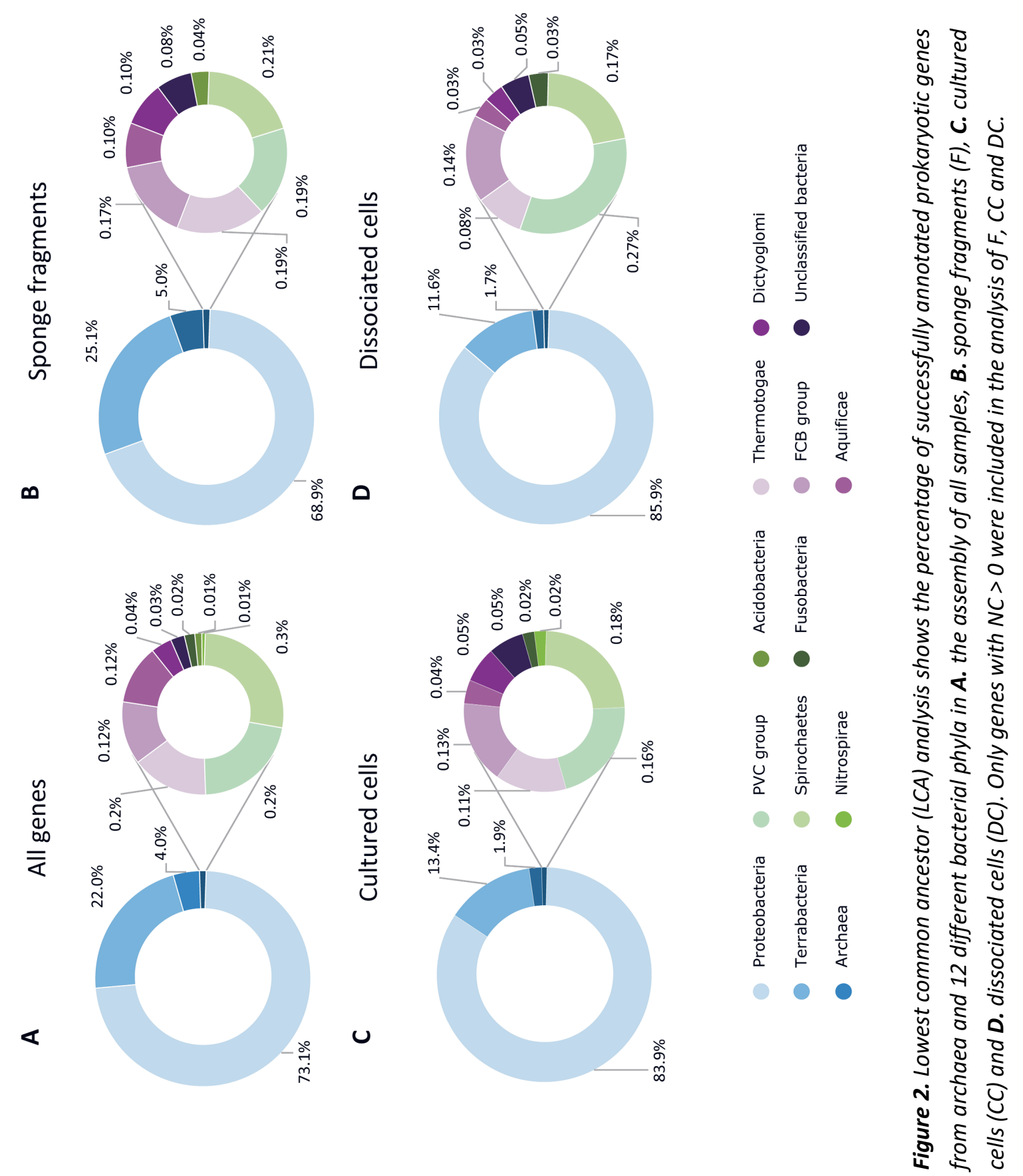
Most prokaryotic genes in the assembly and the different states were assigned to the phylum Proteobacteria (Figure 2). The percentage of genes from Proteobacteria was higher in CC (83.9\%) and DC (85.9\%) (Figure $2 \mathrm{~A}, \mathrm{~B})$ than in the assembly (73.1\%) and F (68.9\%). Conversely, both CC (13.4\%) and DC (11.6\%) contained reduced number of genes from the $2^{\text {nd }}$ most represented phylum, Terrabacteria, compared to the assembly $(22.0 \%)$ and $\mathrm{F}(25.1 \%)$. The $3^{\text {rd }}$ largest proportion of prokaryotic genes was of archaeal origin, and as noted previously this proportion was smaller in CC and DC than in the assembly and F. The remaining genes $(<1 \%)$ were assigned to 9 other bacterial phyla and some originated from unclassified bacteria (Figure 2). Small differences were observed between the 3 states, in particular in 2 phyla that were unique to CC (Nitrospirae) (Figure $2 \mathrm{C}$ ) and $\mathrm{F}$ (Acidobacteria).

\subsubsection{Gene set enrichment analysis}

Compared to F, CC showed significant enrichment of many gene ontology (GO) terms (Table S5). Several of the most significantly enriched GO terms were associated with protein synthesis, such as molecular function (MF) term 'structural constituent of ribosome', Cellular Component term 'ribosome' and Biological Process term 'translation'. Furthermore, GO terms connected with cell structure and (re)organization were enriched in CC, such as 'cytoplasm', 'organelle' and 'intracellular' (Cellular Component), 'plasma membrane organization' and 'cell morphogenesis' (Biological Process). The term 'biosynthetic process' (Biological Process), which contains 'secondary metabolite biosynthetic process' among others, was also enriched in CC/F. There were 3 significantly enriched GO terms in DC/CC: 'signal transduction' (Biological Process), 'enzyme binding' (Molecular Function) and 'cytoskeleton' (Cellular Component). The only GO term significantly enriched in $D C / F$ was oxidoreductase activity (Molecular Function) (Table S5)

In DC/CC, two KEGG pathways regulating cell adhesion were significantly enriched: tight junctions and focal adhesion. The phosphatidylinositol signaling, involved in responses to stimulation of membrane receptors ${ }^{158}$, and phagosome pathways were also enriched (Table S6). Activity of the focal adhesion pathway 
was also significantly different in $C C / F$, as well as regulation of the actin cytoskeleton, ribosome biogenesis and function, and oxidative phosphorylation (OXPHOS) in mitochondria (Table S6). The activity of genes (14) in the latter pathway was also different in DC/F, as well as a few genes (5) in the hypoxiainducible factor 1 (HIF1) signaling pathway, which mediates how cells respond to low oxygen levels ${ }^{159}$.

\subsubsection{Differential expression analysis}

In cultured cells (CC), 1075 genes were expressed at a significantly $(p<0.05)$ higher rate than in sponge fragments $(F)$, while 846 other genes had lower expression levels (Figure 3). Fewer genes were differentially expressed between dissociated cells (DC) and CC (702 up, 146 down) and the least differences were observed between DC and F (159 up, 146 down). Figure 4 shows the log2foldchange (L2FC) in the 100 most significantly differentially expressed genes (DEG) in the comparisons between $C C / F, D C / F$ and $D C / C C$. Based on the results of the GSEA, DEG were most often involved in forming and (re)organizing the cytoskeleton, regulating how cells adhere to each other and to the extracellular matrix (ECM), synthesizing proteins, and producing energy in the form of adenosine triphosphate (ATP).

$\mathrm{DC} / \mathrm{CC}$

702

146

Cultured

cells

1075

846
146

$\mathrm{DC} / \mathrm{F}$
Figure 3. Venn diagram showing the number of genes significantly $(p<$ 0.05) up- (green) and downregulated (purple) between $C C / F, D C / C C$ and $D C / F$. 


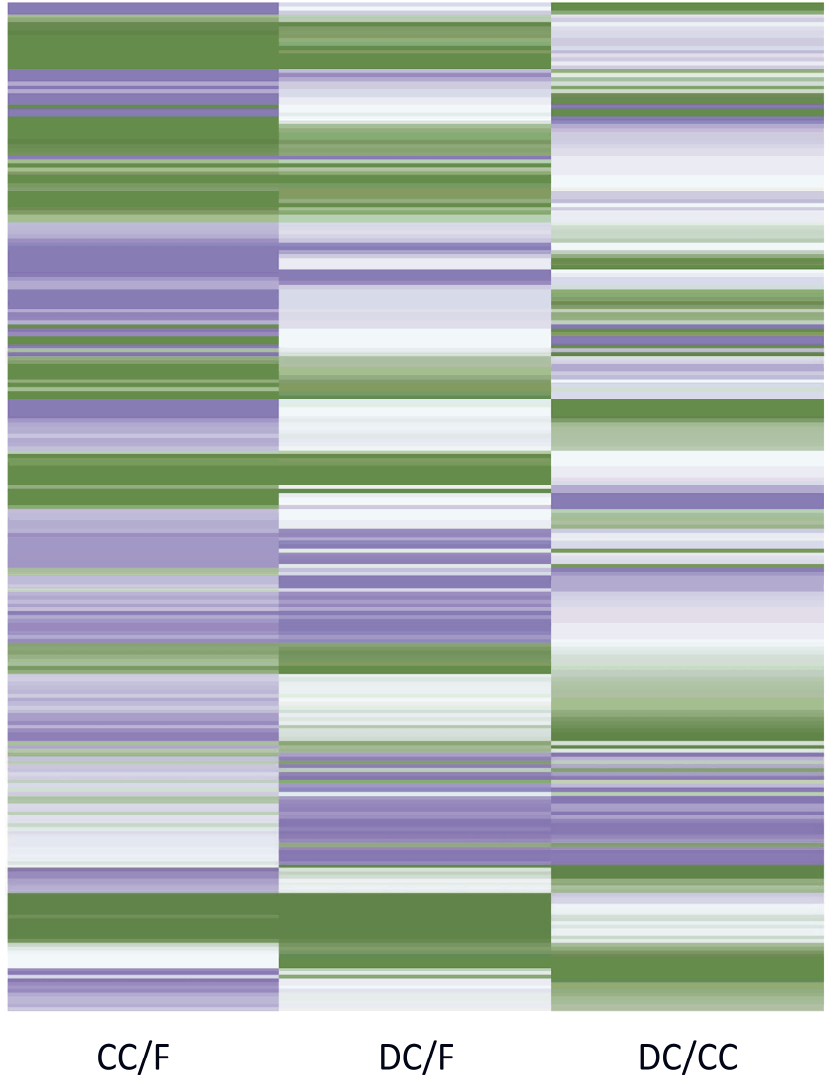

Figure 4. Heatmap of the L2FC in the 100 most significantly (lowest adjusted p-value) DEG between $C C / F, D C / F$ and $D C / C C$. Scale goes from strongly downregulated (L2FC $\leq-6)$ to strongly upregulated ( $L 2 F C \geq 6$ ).

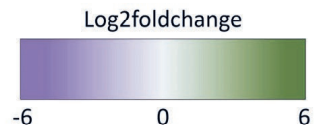

\subsubsection{Cytoskeleton \& adhesion}

Genes encoding proteins that are part of the cytoskeleton, or (re)organize it, were differentially expressed in CC/F and DC/CC (Figure 5). Cytoskeletal actin was strongly upregulated in CC/F with an L2FC of 7.04 ( $p=6.8 \mathrm{E}-04$ ). The normalized expression level of this gene was lower in DC than in CC and higher than in $F$, but not significantly up- or downregulated compared to either $(p=$ 0.90887 and 0.12321, respectively). Multiple filamin transcripts were significantly downregulated in CC/F and upregulated in DC/CC. Filamin-A links actin filaments to the membrane, mediates cell-cell contact, promotes forming adherens junctions ${ }^{160}$, and helps assemble cilia ${ }^{161}$. Many DEG in CC/F and DC/CC were involved in cell adhesion and cell-ECM adhesion was strongly downregulated in CC. Genes at the cell membrane that mediate adhesion such as ankyrin-1 $1^{162}$ (down in CC compared to F and DC) integrin- $\alpha$ PS1 (up in DC/CC) and integrin $\beta$-PS (down in CC compared to $F$ and DC) ${ }^{163}$, components of the ECM 
such as collagen $\alpha 1$ chain (up in $\mathrm{DC} / \mathrm{CC}$ ) and laminin $\beta 1$ (down in CC compared to $\mathrm{F}$ and $\mathrm{DC})^{164}$, cytoskeletal proteins like various myosins ${ }^{165}$ and 2 isoforms of paxillin, which mediates connecting the cytoskeleton to sites of cell adhesion ${ }^{166}$. Some genes involved in cell-cell adhesion were upregulated in $\mathrm{CC} / \mathrm{F}$, such as 3 isoforms of intercellular adhesion molecule $\left(\right.$ ICAM) ${ }^{167}$, possibly as cells attempt to adhere to one-another due to the loss of cell-ECM adhesion.

Cytoplasmic dynein heavy chains (DYNCH) are part of the dynein motor and required to assemble and move cilia ${ }^{168}$, as well as vesicle-mediated transport ${ }^{169}$, 170, , 171. Multiple isoforms of this gene were strongly downregulated in CC, compared to both F and DC (Figure 5). Conversely, an isoform of cytoplasmic dynein light chain (DYNCL), which regulates how vesicles and organelles are transported along microtubules and is also involved in forming the mitotic spindle ${ }^{172}$, was upregulated in CC compared to $F$ and DC. Both $F(p=0.0019$, L2FC $=-6.21)$ and dissociated cells $(p=9.87 \mathrm{E}-05, \mathrm{~L} 2 \mathrm{FC}=7.17)$ expressed girdin at $\mathrm{a}$ significantly higher level than in CC. Girdin is the short name for guanine nucleotide-binding (G) protein $\alpha$ subunit $(G \alpha)$-interacting vesicle-associated protein (GIV) which binds guanine nucleotide-binding (G) proteins, stimulating cells to assemble cilia, migrate and not proliferate ${ }^{173}$.

\subsubsection{Protein synthesis \& energy production}

In both CC and DC, major genes that form the electron transport chain and produce adenosine triphosphate (ATP) production through oxidative phosphorylation were expressed at higher levels than in $F$, such as cytochrome $c$ oxidase subunit I $(p=0.00019$ and 0.014$)$ and III $(p=0.00091 \text { and } 0.018)^{174}$, and $\mathrm{NADH}$ dehydrogenase subunit $4(p=0.00011$ and 0.023$)$ and $5(p=0.046$ and $0.026)^{175}$. In $C C$, many proteins that are part of the large (60S) and small (40S) ribosomal subunits were significantly higher expressed than in $F$, as were genes that promote ribosome biogenesis such as ribosome biogenesis factor BMS1 $1^{176}$ $(p=0.034)$, eukaryotic initiation factor 6 (EIF6)177, 178, 178, 179, $179(p=$ $0.044)$, and 1 isoform of exportin-1180, 181, 181 ( $p=0.023)$, while another isoform of exportin-1 was downregulated $(p=0.00031)$. 
Cytoskeleton \& adhesion

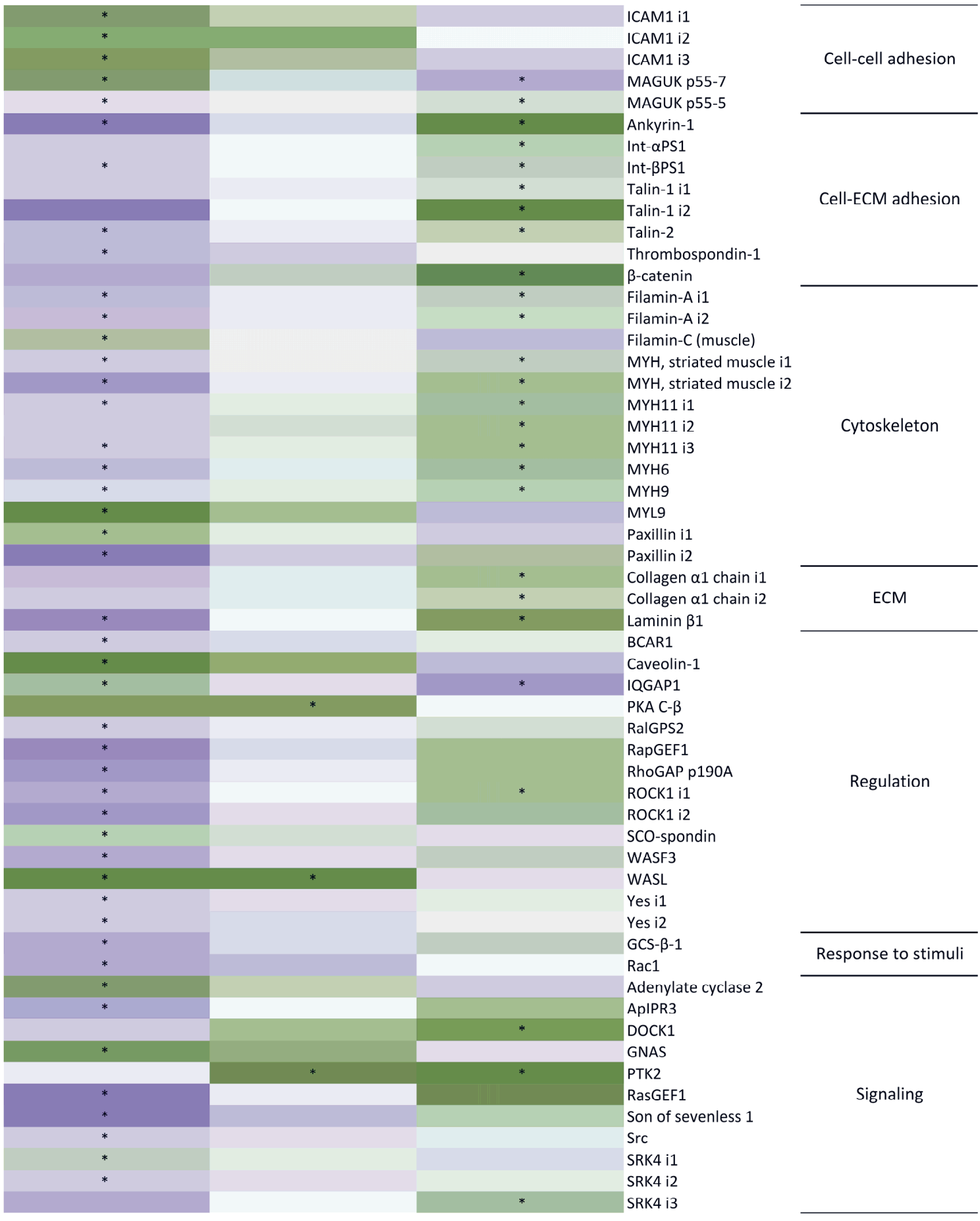

$\mathrm{CC} / \mathrm{F} \quad \mathrm{DC} / \mathrm{F} \quad \mathrm{DC} / \mathrm{CC}$

Figure 5. Heatmap of the L2FC in genes that are involved in or regulate the cytoskeleton, ECM and/or cell adhesion and are differentially expressed between $C C / F, D C / F$ and $D C / C C$. Scale goes from strongly downregulated ( $L 2 F C \leq-6)$ to strongly upregulated ( $L 2 F C \geq 6$ ). Asterisks

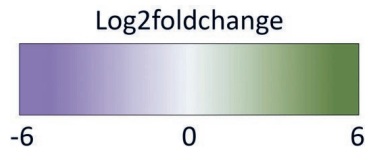
indicate which differences are significant ( $p<0.05)$. 


\subsubsection{Endocytosis \& metabolism}

One of 2 isoforms of clathrin heavy chain, essential for phagocytosis and other forms of endocytosis ${ }^{177,178,178}$, was downregulated in CC compared to both $\mathrm{F}$ and DC, while another was downregulated compared to F. Various other genes involved in vesicle-mediated transport were also expressed at lower levels, including dyneins ${ }^{169,169-171,171}$, phosphoinositide 3-kinases (PI3K) which stimulate phagocytosis ${ }^{179}$ and its downstream target Rac1 ${ }^{180}$. Conversely, genes that inhibit endocytosis were upregulated in CC compared to F and DC, such as phosphatidylinositol 3,4,5-trisphosphate 5-phosphatase 2 (INPPL1) ${ }^{180}$ and SH3 domain-containing kinase-binding protein 1 (SH3KBP1) ${ }^{181,182,182}$. A number of components of the endosomal sorting complex required for transport III (ESCRT III) were expressed at higher levels in CC than F or DC, possibly because ESCRT III is also required for cytokinesis ${ }^{183}$.

Cultured cells also metabolized fat differently, for example, the extracellular gastric triacylglycerol lipase ${ }^{184}$ was upregulated in $C C / F(p=0.00066)$. While this gene was also upregulated in $D C / F$, this difference was not significant $(p=0.193)$. Multiple isoforms of fatty-acid synthesis enzyme acetoacetyl-CoA synthetase ${ }^{185}$ were upregulated in $\mathrm{CC}$ compared to $\mathrm{F}$ and $\mathrm{DC}$, although 1 isoform was expressed significantly higher in DC/CC ( $p=4.55 \mathrm{E}-06)$. Short/branched $(p=0.00040)$ and medium chain $(p=0.0029)$ fatty acid beta oxidation were downregulated in CC compared to $F$, expression was not significantly different in DC/CC or DC/F. The same was observed for glycogen catabolic enzyme glycogen phosphorylase ${ }^{186}$, which was lower in $\mathrm{CC}$ than in $\mathrm{F}(\mathrm{p}=3.87 \mathrm{E}-05)$ but not different in $\mathrm{DC}$ from either. Both CC ( $p=0.028)$ and DC $(p=0.00012)$ had upregulated isoforms of the same gene, Rab32, which helps assemble melanin-synthesizing melanosomes ${ }^{187}$ and could be involved in producing the dark pigment observed in sponge cell cultures $^{136}$, which is suspected to be melanin. 
Vesicle-mediated transport

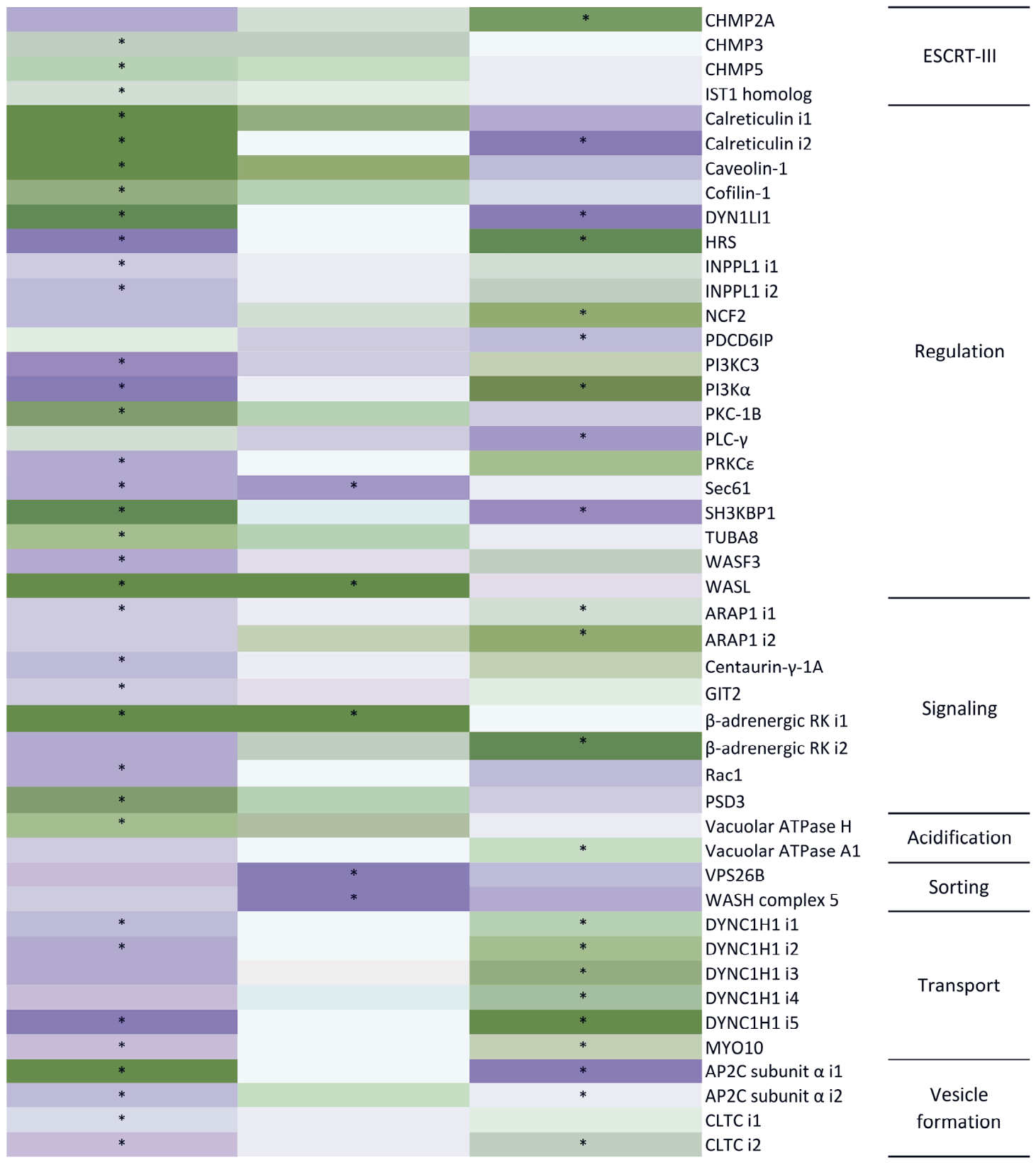

$\mathrm{CC} / \mathrm{F}$

$D C / F$

$\mathrm{DC} / \mathrm{CC}$

Figure 6. Heatmap of the L2FC in genes that are involved in or regulate vesicle-mediated transport and are differentially expressed between $C C / F, D C / F$ and $D C / C C$. Scale goes from strongly

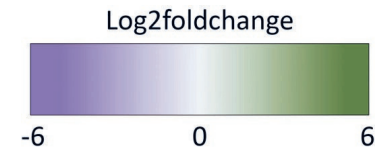
downregulated (L2FC $\leq-6)$ to strongly upregulated ( $2 F C \geq 6$ ). Asterisks indicate which differences are significant $(p<0.05)$. 


\subsubsection{Cell division \& apoptosis}

Many genes that drive and regulate the cell cycle, such as cyclins and cyclindependent kinases, were highly expressed at the same level in CC, F and DC samples. This included $G 1 / S$ phase cyclins $D$ and $E$, and the associated cyclindependent kinases (CDK) 2, CDK4 and CDK6, S/G2 phase cyclin A1 and CDK1, and $M$ phase cyclin $B^{188}$. No transcripts encoding tumor suppressor $p 53$, caspase 3 and apoptosis regulator $\mathrm{BAX}$, all of which negatively regulate cell cycling and promote apoptosis ${ }^{189}$, were highly or differentially expressed in any sample. No transcripts were annotated as the highly conserved CDK inhibitor 2A (p21) ${ }^{190}$, suggesting that p21 was not expressed enough to be detected using our methods. Transcripts of genes that p21 interacts with, such as p21-activated

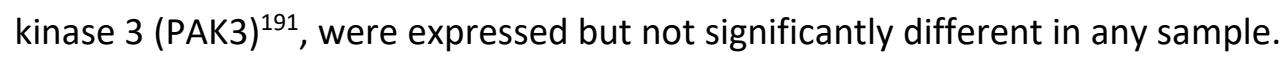
Although cell cycling and apoptosis pathways were not significantly enriched, some genes that are either part of or regulate these processes were differentially expressed (Figure 5). For example, 1 isoform of mitotic checkpoint protein BUB3 ${ }^{192,193,193}$ was significantly upregulated in $C C / F$, while another isoform was downregulated. In both $C C / F$ and $D C / F$, genes involved in the bacterial cell cycle were downregulated, suggesting fewer actively dividing bacteria in these samples. E3 ubiquitin-protein ligase COP1, which ubiquinates p53, causing it to be degraded ${ }^{194}$, was upregulated in CC/F, and its counterpart that prevents $p 53$ from being degraded by deubiquitinating it, called ubiquitin carboxyl-terminal hydrolase 7 (USP7) 195, 196, 196 was downregulated. A number of cathepsins were significantly upregulated in $\mathrm{CC} / \mathrm{F}$, which seems counterintuitive at first since cathepsins stimulate apoptosis ${ }^{197}$. However, the role of cathepsins in regulating apoptosis is more complex, as these proteins are also upregulated in various types of tumor cells ${ }^{198,199,199}$. Three isoforms of $60 \mathrm{kDa}$ heat-shock proteins (HSP60), which ensure proteins are properly folded to prevent apoptosis under stress ${ }^{200}$ and also promote tumor cell survival ${ }^{201}$ were differentially expressed ( 2 up, 1 down) in both CC/F and DC/F. 
Cell cycling, DNA repair \& apoptosis

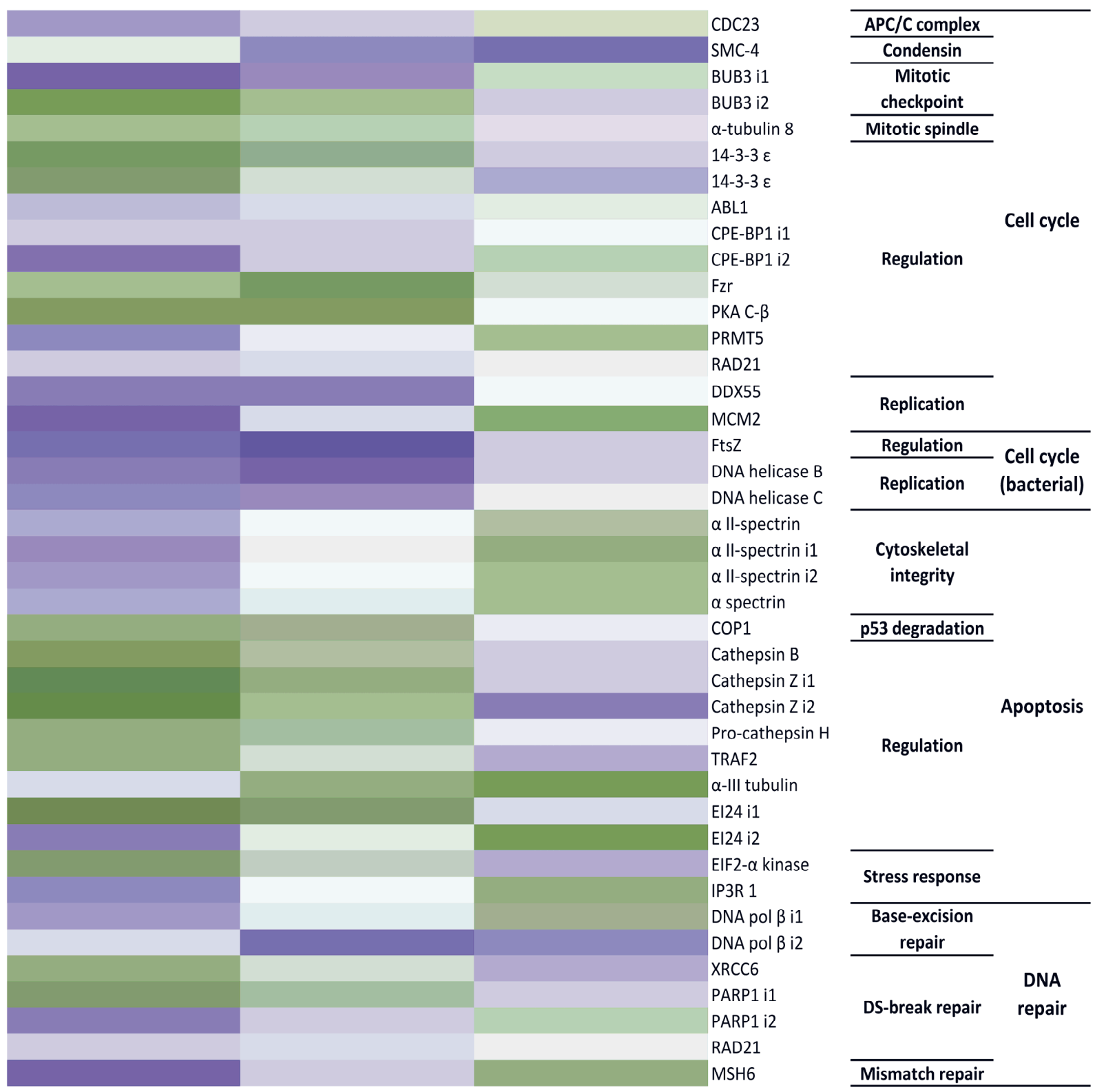

$\mathrm{CC} / \mathrm{F}$

$D C / F$

$\mathrm{DC} / \mathrm{CC}$

Figure 7. Heatmap of the L2FC in genes that are involved in or regulate the cell cycle, apoptosis or differentiation and are differentially expressed between $C C / F, D C / F$ and $D C / C C$. Scale goes from strongly downregulated ( $L 2 F C \leq-6$ ) to strongly upregulated ( $L 2 F C \geq 6$ ). Asterisks

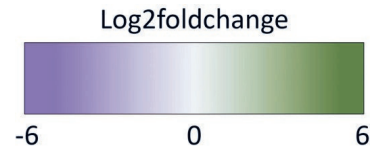
indicate which differences are significant $(p<0.05)$. 


\subsubsection{DNA repair}

All major DNA repair pathways were expressed in all samples, including baseexcision repair proteins DNA glycosylase $202,203,203$ and DNA ligase $3^{204}$, and double-strand break repair proteins RAD50 and MRE11 ${ }^{205}$. DNA damage detectors and DS-break repair proteins poly [ADP-ribose] polymerases PARP1 ${ }^{206}$, $\mathrm{PARP}^{207}$ and PARP3 ${ }^{208}$ were also ubiquitously expressed. The only 3 DEG were 2 isoforms of PARP1 (1 up- and 1 downregulated in CC/F) and 1 isoform of X-ray repair cross-complementing protein 6 (XRCC6) ${ }^{209}$ (up in CC/F) (Figure 7).

\subsubsection{Longevity, stemness \& differentiation}

Only partial transcripts of $G$. barretti telomerase reverse transcriptase (TERT), responsible for lengthening telomeres, were found in the assembly. Partial TERT transcripts were expressed in all samples with an average total NC of all TERT transcripts was $42.6,36.38$, and 23.21 for DC, CC and F, respectively. This supports previous observations that TERT was detected in dissociated cells of marine sponge Axinella corrugata using immunohistochemistry ${ }^{59}$ and telomerase was active in Suberites domuncula and Geodia cydonium ${ }^{82}$ sponges, and cell aggregates (primmorphs) of $S$. domuncula ${ }^{89}$. Genes that regulate TERT and maintain telomeres were also ubiquitously expressed, including tripeptidylpeptidase 1 (TPP1) that recruits TERT to telomeres ${ }^{210}$ and telomerase Cajal body protein 1 (TCAB1) that matures telomerase RNA component (TERC) in nuclear Cajal bodies ${ }^{211}$. PinX1 inhibits TERT when highly expressed ${ }^{212,213,213}$, but is needed to maintain telomeres ${ }^{212}$. Tankyrase inhibits telomeric repeat-binding factor 1 and 2 (TRF-1 \& 2) which make telomeres inaccessible to TERT ${ }^{214}$.

A number of genes involved in deciding cell fate were differentially expressed in $\mathrm{CC} / \mathrm{F}$ and $\mathrm{DC} / \mathrm{CC}$. For example, 2 isoforms of activin receptor $2 \mathrm{~A}$, which stimulates various mammalian cell types to differentiate 215,216 , were downregulated in CC/F and upregulated in $\mathrm{DC} / \mathrm{CC}$, as were genes involved in Wnt signaling which also causes cells to differentiate ${ }^{217}$. Fibroblast growth factor receptor 2 (FGFR2) had 2 upregulated isoforms in CC/F, one of which was also downregulated in DC/CC. FGFR2 activates multiple signaling pathways that promote cell cycling ${ }^{218}$ and is often constitutively active in skin and bone cancers ${ }^{219}$. 


\subsection{Discussion}

We investigated how genes are expressed differently between sponge fragments, dissociated and cryopreserved cells, and cells cultured in OpM1 medium. Recently, we developed the first continuous sponge cell line for the deep-sea boreal sponge $G$. barretti (Hesp et al., in prep), with the cells cultured in the OpM1 medium (Munroe et al. in prep). Studying cells in culture can reveal how cellular processes are regulated in sponge cells, like how they proliferate, prevent apoptosis, respond to stress or take up nutrients.

We sequenced RNA isolated from cells in these 3 different states for $3 \mathrm{G}$. barretti individuals, assembled the transcriptome de novo, assessed the taxonomic origin of the assembled genes, and performed differential expression analysis (DEA) and gene set enrichment analysis (GSEA) to better understand how sponge cells respond to the stress of being dissociated and cryopreserved, and adapt to cell culture. Since DC contain the same cell types as F, and DC are not exposed to nutrients after being thawed, enriched gene functions and pathways are probably actively up- or downregulated in DC as the cells respond to being dissociated and cryopreserved. Culturing sponge cells in OpM1 results in more differentially expressed genes which may be due to adaptation of cells, selection of certain cell types, or both. This suggests that some changes in which genes are expressed may be relative, rather than actively adapted by the cells.

\subsubsection{Lowest common ancestor analysis}

Relatively few differences were observed between the number of prokaryotic genes in the 3 different states. The lower percentage of prokaryotic genes in the samples compared to the full assembly is likely because only genes with NC $>0$ were analyzed for the samples. This may indicate that some microbes, possibly (endo)symbionts, remain active when cells are cultured in OpM1, despite the presence of antibiotics. Another possibility is that $G$. barretti carries and expresses prokaryotic genes obtained from its symbionts through horizontal gene transfer, as was found for $A$. queenslandica ${ }^{220}$. Some differences were observed between the assembly and the 3 states, such as the reduced number of archaeal genes in CC and DC compared to the assembly and F. This could 
indicate that a portion of the archaeal genes belonged to non-symbiotic archaea, or that archaea are more likely to be removed by washing steps performed when the cells are dissociated and cryopreserved. Many of the phyla found in our assembly were well-represented when gene sequences from sponge metatranscriptomes were analyzed to identify putative sponge-specific bacteria, including Proteobacteria, Spirochaetes, Nitrospirae, Acidobacteria, Actinobacteria, Fusobacteria and Chloroflexi ${ }^{221}$ 222. The 2 phyla unique to $F$ (Acidobacteria) and CC (Nitrospirae) could suggest that some bacteria cannot transition easily into culture with the sponge cells, while others may benefit from their new environment. Observing changes in the community profile of $G$. barretti cell cultures over multiple passages through $18 \mathrm{~S}$ and $16 \mathrm{~S}$ sequencing could provide a closer look at (obligate) microbial symbionts.

\subsubsection{Gene set enrichment analysis}

Analyzing which gene functions are enriched under certain conditions can provide a broad picture of what is happening in the cells. The most functions are enriched in $C C / F$, suggesting the major changes are needed to adjust to cell culture. Losing the support of an ECM also means CC must maintain their own shape, which requires them to reorganize their cytoskeletons, likely leading to the enriched GO-terms 'plasma membrane organization' and 'cell morphogenesis' (Biological Process), and KEGG pathway 'regulation of actin cytoskeleton'. Cells divide rapidly in OpM1, and therefore increase the number of ribosomes to synthesize proteins, produce lipids for cell membranes, make cytoskeletal changes required for cells to separate, and construct the organelles each sister-cell will need to survive. Another enriched gene function in CC/F was 'biosynthetic process', which includes 'secondary metabolite biosynthetic process' indicating that $G$. barretti cells may produce either more or less secondary metabolites in vitro. Either way, examining which genes are up- or downregulated may provide clues regarding the pathways that synthesize these metabolites and how they could be targeted to become (more) active. DC/F showed few differences, the only significantly enriched function is oxidoreductase activity. This could be linked to cells responding to oxidative 
stress $^{223}$ after being exposed to air during dissociation, or a lack of oxygen. No gene functions were significantly enriched in DC/CC.

The analysis of how KEGG pathways are differentially regulated between the 3 states shows that there are major changes in gene expression in DC/CC. The pathways that regulate how cells interact with and adhere to their surroundings change as CC lose contact with an ECM. Furthermore, CC downregulate phagocytosis genes, indicating they may rely less on phagocytosing nutrients, as many are present in soluble form in OpM1 medium that can either diffuse or be transported into the cells by enzymes in the plasma membrane. Pathways that organize the actin cytoskeleton and regulate ribosomes also show changed activity in CC compared to $F$, as in the GO-term GSEA. Both in DC/F and CC/F oxidative phosphorylation (OXPHOS) is upregulated, possibly responding to changes in the intra- and extracellular redox state, which regulate mitochondrial activity $^{223}$. This also concurs with the enriched GO-term oxidoreductase activity and altered activity of genes in the HIF1 pathway in DC/F. However, twice as many OXPHOS genes were upregulated in CC (28 up, 1 down) than in DC (14 up), indicating OXPHOS is upregulated in CC for another reason, possibly to meet the increased ATP demand in the rapidly dividing cells.

\subsubsection{Differential expression analysis}

The number of genes up- or downregulated between states varied strongly. Most genes were differentially expressed in CC/F, followed by DC/CC, and finally $D C / F$. Evidently, the cells undergo many changes between proliferating in 3 dimensions, supported by an ECM, and as cells in vitro. Some of those changes are already occurring in DC, leading to a smaller difference in $D C / C C$. The difference in $\mathrm{DC} / \mathrm{F}$ is small, suggesting that being dissociated and cryopreserved does not strongly affect the cells, which is substantiated by similar viabilities observed in cells before and after cryopreservation ${ }^{54,54,102}$. A schematic representation of the differences between cells in an intact sponge and in cell culture is shown in Figure 8, while Figure S1 shows microscopic images of $G$. barretti cells in culture. 


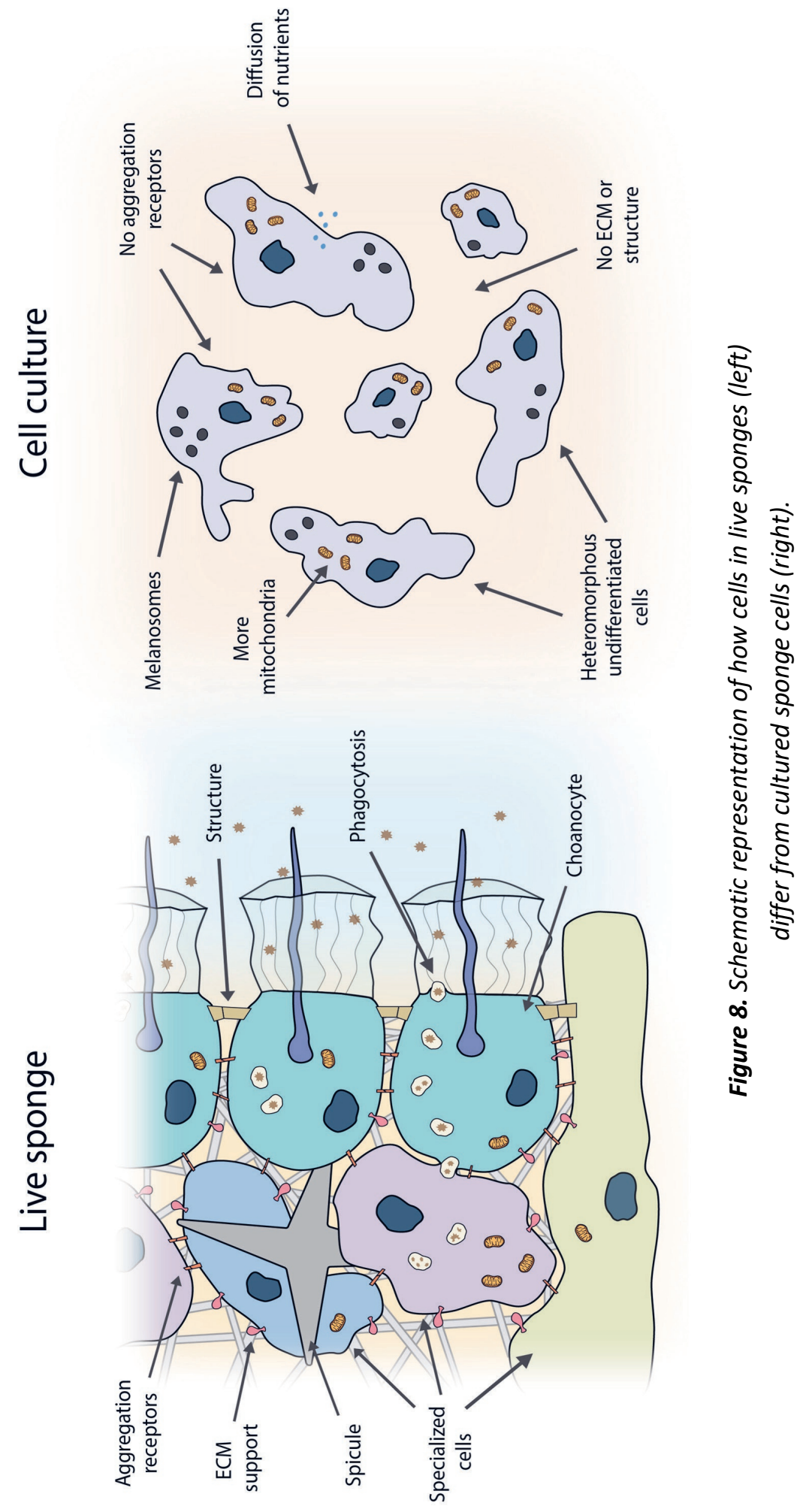




\subsubsection{Cytoskeleton, extracellular matrix \& cell adhesion}

The strong upregulation of actin in CC, compared to F, is likely due to the important role actin cytoskeleton dynamics play in eukaryotic cell cycling ${ }^{224}$. Changes in cytoskeletal organization in dissociated and dividing cells compared to tissue could also be partly due to the loss of support of the ECM, requiring the cells to reorganize their cytoskeleton to maintain their shape. For example, cells require girdin to respond to pressure changes by forming actin stress fibers at the cell membrane 225 , as well as filopodia to probe the surrounding area for other cells ${ }^{226}$ and cilia to move towards these cells to reaggregate ${ }^{173}$. Girdin is downregulated in $\mathrm{CC} / \mathrm{F}$, possibly because $\mathrm{CC}$ no longer need to interact with the $\mathrm{ECM}$ and other cells, or to respond to slight differences in pressure due to the flow of ocean water. The cells cultured in OpM1 medium form fewer aggregates and survive without an ECM (Figure 8, S1), either because the cells adapt or culture conditions select for a cell type that is less likely to aggregate or both.

Dyneins form a molecular motor that travels down microtubules and are highly conserved in eukaryotes ${ }^{168,168,170,170,171,227,227,228,228}$. Organisms with ciliated cells use the dynein motor to move around ${ }^{168,168,227,228,228}$ and have many specialized isoforms of its components. For example, 14 different DYNHC mRNAs were found in sea urchin embryos, at least 11 of which embryonic cells differentially expressed after their cilia were removed ${ }^{228}$. Dissociated cell suspensions are a mixture of all sponge cell types, including choanocytes, but no cilia or moving cells are observed in G. barretti cell cultures (Hesp et al., unpublished data). Our data indicate that either CC dedifferentiate and downregulate specialized cell structures like cilia, or culture conditions select for undifferentiated cells.

Sponge ECMs resemble those of higher metazoans, consisting of collagen (spongin) and other protein polymers, and proteins that ensure cells adhere to the ECM and each other $229,230,230$. A plethora of differentially expressed ECMrelated genes demonstrates the extent of the changes that cells undergo to survive without the support of an ECM (Figure 4). Sponge cells possess specialized species-specific receptors that strongly bind to the soluble sponge aggregation factor (AF), allowing them to reaggregate and form a new sponge ${ }^{229 \text {, }}$ 
${ }^{231-234}$. The aggregation receptor (AR) of $G$. cydonium, a member of the same genus as $G$. barretti, was the first to be isolated ${ }^{234}$ and characterized ${ }^{232}$. We identified the partial AR of $G$. barretti, which contains 17 SRCR domains and is highly similar to $G$. cydonium's AR, as well as human vascular ECM receptor DMBT1, which regulates how cells adhere and migrate during angiogenesis ${ }^{235}$. The ARs of both sponges are so similar to DMBT1 that we conclude these genes are orthologs that evolved to fulfill different but related functions in sponges and higher metazoans. The AR of $G$. barretti was dowregulated in $\mathrm{CC} / \mathrm{F}$ ad upregulated in DC/CC, further indicating that $G$. barretti cells can survive as single cells and do not need to aggregate to divide.

\subsubsection{Metabolism \& energy production}

OXPHOS genes are upregulated in both DC/F and CC/F. While in both DC and CC these genes may be more active to respond to changes in the redox state, the number of upregulated genes in CC is larger, likely to produce energy to grow and divide. Downregulated phagosome genes like CLTC show that CC are less reliant on phagocytosis and likely obtain nutrients by diffusion or transport across the cell membrane (Figure 9). CC downregulated glycogen phosphorylase ${ }^{186}$, suggesting they used less glucose stored in the form of glycogen, probably because glucose and other energy sources are readily available in the medium, Likely for the same reason, CC downregulated fatty acid $\beta$-oxidation, breaking down fewer fats to produce ATP, and upregulated genes that synthesize lipids to make membranes for new cells and organelles. All these observations indicate that OpM1 medium provides enough nutrients for the cells to divide without phagocytosing bacteria or particulate organic matter and without using their energy reserves. Both DC and CC upregulated the Rab32 gene required for melanosome assembly ${ }^{187}$, which may be related to dark pigments present in cultured sponge cells as inclusions and secreted into the medium ${ }^{136}$. Since $G$. barretti is naturally cream-colored and does not produce such pigments, it is possible the pigment is a response to being exposed to light. Melanin is also used as an antioxidant by both animals ${ }^{236,237}$ and microbes ${ }^{129,238-240}$. Whether this pigment is indeed melanin, and why and how it is produced, will need to be investigated further. 


\subsubsection{Cell division, stress response \& apoptosis}

Genes required for cells to divide, such as cyclins and CDKs, were expressed ubiquitously in F, DC and CC. This suggests that cells in the adult sponge were proliferating when the samples were collected, or that sponge cells around the cuts responded quickly to repair the sponge's body. Sponges can remold and regenerate their body when damaged, and even use this to propagate asexually ${ }^{21}, 23$. That dissociated and cryopreserved sponge cells express cell cycling genes shows they are viable and able to divide, and that the shift towards apoptosis ${ }^{57}$ observed in sponge cell cultures is most likely because cells do not have the nutrients required to start dividing again. It is also consistent with the high viability observed in dissociated sponge cells of multiple species before ${ }^{102}$, 136 and after being cryopreserved ${ }^{54,102}$. These conclusions are further supported by the low expression levels of pro-apoptotic p53, caspase 7, and BAX in all samples. Some stress-induced genes were upregulated in DC, like alternate oxidase, which provides alternate respiration when the cytochrome pathway is restricted or in response to low temperatures ${ }^{241}$, such as during cryopreservation.

\subsubsection{Telomere length \& maintenance}

Every time the genome is replicated in eukaryotes, some base pairs are lost at the $3^{\prime}$ end of each DNA strand. Stretches of repeated DNA sequences, called telomeres, are present at the ends of chromosomes. Short fragments of telomeres are sacrificed with each replication to prevent genomic DNA from being lost. Telomerase reverse transcriptase (TERT) is the catalytic subunit of the ribonucleoprotein complex responsible for lengthening telomeres ${ }^{242,243}$. Its activity level determines how many times a cell can divide before the telomeres run out and cells become senescent or apoptotic. In most higher eukaryotes, TERT transcription is strongly regulated and present at very low to undetectable levels in most cells, except in those required to divide more often, like stem cells and germline cells. TERT is also well-known as an oncogene, as it allows cells to replicate limitlessly when overexpressed ${ }^{244,245}$. Our results show that $G$. barretti cells express TERT constitutively, confirming previously observed TERT activity in sponge cells $s^{59,82,89}$. Despite active TERT, telomeres have a limited length in $S$. 
domuncula cells ${ }^{89}$, and cancerous sponge cells have not been reported ${ }^{246}$. While the lengths of telomeres in other sponge species have not yet been determined, this suggests telomere length is regulated in a different manner, for example by expressing proteins that either negatively, like PinX1213, or positively, like tankyrase ${ }^{214}$, affect telomerase activity. It has been stipulated that sponges may be naturally immortal, as they are the longest living animals ${ }^{247,248}$ that reach ages of hundreds or even thousands of years old. The oldest recorded sponge is a siliceous deep-sea Monorhaphis chuni specimen believed to be around 11,000 years old, based on analysis of its spicules' oxygen isotope composition and magnesium/calcium ratios ${ }^{247}$. With the recent progress in developing cell lines ${ }^{136}$ (Hesp et al., in prep), it will be interesting to discover if sponge cell lines are immortal by nature. Examining how telomere length changes over time in cultured $G$. barretti cells could shed more light on this question.

\subsubsection{DNA repair}

The first mammalian cell lines such as $\mathrm{HeLa}^{70}$ and $\mathrm{HT}-29^{249}$ were derived from cancerous cells. Sponges do not seem to develop tumors ${ }^{246}$, which is among the reasons developing a sponge cell line has been so challenging. Because of their long lifespans and potential immortality, it seems likely that sponges possess potent mechanisms to repair damage to their DNA and prevent their cells become cancerous or accumulate deleterious mutations. Tethya lyncurium sponges fully repaired single-stranded breaks in their genome, 3 weeks after being exposed to the mutagenic combination of benzo[a]pyrene and light. DNA synthesis was also observed during this time ${ }^{250}$. While genes from known DNA repair pathways were expressed in all samples, it is difficult to determine whether this could lead to more efficient repair of DNA damage. Furthermore, it is possible that sponges use mechanisms that are adapted from known or novel pathways. In vitro cell cultures can provide an ideal model system to study how sponges respond to and repair DNA damage. 


\subsection{Conclusion}

Our results provide unprecedented insight into how cellular processes are regulated in sponge cells and help us understand how sponge cells adjust to survive in cell culture. The most dramatic changes seemed required to cope with losing support of an ECM. We demonstrated that sponge cells can survive these changes and continue to divide. Whether this is because cells actively adapt to their new surroundings or because culture conditions favor a specific cell type, needs to be investigated further. The cells also obtain and metabolize nutrients differently, reducing phagocytosis and focusing on producing lipids and other building blocks needed to proliferate. A closer look at differentially expressed genes with the enriched gene function 'biosynthesic process' may provide clues as to what pathways are involved in synthesizing secondary metabolites and how cells can be stimulated to produce higher levels of such metabolites. Our results also confirm previous reports of active TERT in sponge cells, raising anew the question whether sponges are naturally immortal. We show that intact sponges, and dissociated and cryopreserved cells express genes to progress through the stages of the cell cycle, at levels like those of cultured cells. How sponge cells respond to and repair DNA damage, and whether their methods are superior to those of other animals, still has to be uncovered. 


\subsection{Supplementary material}

Table S1. Quality control

\begin{tabular}{|c|c|c|c|}
\hline Sample & Raw reads & Clean reads & $\%$ \\
\hline GB1CC & 24650198 & 24109589 & 97.81 \\
\hline GB1DC & 24016870 & 23610697 & 98.31 \\
\hline GB1F & 25835538 & 25601904 & 99.10 \\
\hline GB2CC & 20138146 & 19784587 & 98.24 \\
\hline GB2DC & 28099283 & 27912894 & 99.34 \\
\hline GB2F & 28526078 & 28171053 & 98.76 \\
\hline GB3CC & 26637859 & 26389699 & 99.07 \\
\hline GB3DC & 23586331 & 23100717 & 97.94 \\
\hline GB3F & 27978577 & 27714027 & 99.05 \\
\hline Total & 229468880 & 226395167 & 98.66 \\
\hline
\end{tabular}

Table S3. Completeness assessment

\begin{tabular}{|l|l|l|}
\hline Orthologs & $\#$ & $\%$ \\
\hline Queried & 978 & 100.00 \\
\hline Complete & 912 & 93.25 \\
\hline Complete + partial & 948 & 96.93 \\
\hline Missing & 30 & 3.07 \\
\hline
\end{tabular}

Table S2. Assembly statistics

\begin{tabular}{|l|l|}
\hline & \# basepairs \\
\hline Min length & 201 \\
\hline Mean length & 1004 \\
\hline Median length & 601 \\
\hline Max length & 30018 \\
\hline N50 & 1595 \\
\hline N90 & 424 \\
\hline Total nucleotides & 205690798 \\
\hline & \# Transcripts \\
\hline $200-500 b p$ & 84303 \\
\hline $500-1 \mathrm{kbp}$ & 56142 \\
\hline $1 \mathrm{k}-2 \mathrm{kbp}$ & 38696 \\
\hline$>2 \mathrm{kbp}$ & 25798 \\
\hline Total & 204939 \\
\hline Unigenes & 204668 \\
\hline
\end{tabular}

Table S4. Annotation statistics

\begin{tabular}{|l|l|l|l|l|l|}
\hline Annotated in & \# Unigenes & $\%$ & Species NR hits & \# Unigenes & $\%$ \\
\hline NR & 111314 & 54.39 & Amphimedon queenslandica & 45458 & 40.84 \\
\hline NT & 38326 & 18.73 & Nephila clavipes & 4436 & 3.99 \\
\hline KO & 52822 & 25.81 & Homo sapiens & 2747 & 2.47 \\
\hline Swissport & 92816 & 45.35 & Stylophora pistillata & 2368 & 2.13 \\
\hline PFAM & 28693 & 14.02 & Exaiptasia pallida & 2097 & 1.88 \\
\hline GO & 17173 & 8.39 & Orbicella faveolata & 2041 & 1.83 \\
\hline KOG & 56421 & 27.57 & Other & 52154 & 48.69 \\
\hline All databases & 3142 & 1.54 & & & \\
\hline$\geq 1$ database & 122277 & 59.74 & & & \\
\hline
\end{tabular}


Table S5. Enriched GO-terms in each comparison (CC/F, DC/CC and CC/F)

\begin{tabular}{|c|c|c|l|c|c|}
\hline Comparison & Category & GO-term & Description & padjust & \#DEG \\
\hline CC/F & MF & GO:0003735 & structural constituent of ribosome & $7.24 \mathrm{E}-12$ & 41 \\
\hline & MF & GO:0005198 & structural molecule activity & $9.15 \mathrm{E}-12$ & 43 \\
\hline & CC & GO:0005840 & ribosome & $1.04 \mathrm{E}-11$ & 41 \\
\hline & BP & GO:0006412 & translation & $2.61 \mathrm{E}-09$ & 43 \\
\hline & CC & GO:0005737 & cytoplasm & $4.42 \mathrm{E}-08$ & 58 \\
\hline & CC & GO:0043226 & organelle & $6.42 \mathrm{E}-05$ & 66 \\
\hline & CC & GO:0005622 & intracellular & $7.57 \mathrm{E}-05$ & 83 \\
\hline & BP & GO:0009058 & biosynthetic process & $7.57 \mathrm{E}-05$ & 82 \\
\hline & CC & GO:0032991 & protein-containing complex & $7.57 \mathrm{E}-05$ & 60 \\
\hline & CC & GO:0005623 & cell & 0.000141 & 84 \\
\hline & BP & GO:0007009 & plasma membrane organization & 0.000507 & 3 \\
\hline & BP & GO:0006091 & generation of precursor & 0.0038 & 10 \\
& & & metabolites and energy & & \\
\hline & MF & GO:0003723 & RNA binding & 0.006108 & 22 \\
\hline & MF & GO:0019899 & enzyme binding & 0.00853 & 10 \\
\hline & BP & GO:0061024 & membrane organization & 0.010705 & 3 \\
\hline & BP & GO:0000902 & cell morphogenesis & 0.022828 & 3 \\
\hline & MF & GO:0019843 & rRNA binding & 0.028872 & 3 \\
\hline DC/CC & BP & GO:0007165 & signal transduction & 0.010172 & 15 \\
\hline & MF & GO:0019899 & enzyme binding & 0.010172 & 6 \\
\hline CC & GO:0005856 & cytoskeleton & 0.010172 & 7 \\
\hline & MF & GO:0016491 & oxidoreductase activity & $1.67 \mathrm{E}-07$ & 21 \\
\hline & & &
\end{tabular}

Table S6. Enriched KEGG pathways in each comparison (CC/F, DC/CC and DC/F)

\begin{tabular}{|c|c|l|c|c|}
\hline Comparison & KEGG ID & Description & padjust & \#DEG \\
\hline CC/F & ko03010 & Ribosome & $4.04 \mathrm{E}-09$ & 62 \\
\hline & ko00190 & Oxidative phosphorylation & 0.004181 & 29 \\
\hline & ko04810 & Regulation of actin cytoskeleton & 0.009105 & 25 \\
\hline & ko04510 & Focal adhesion & 0.040284 & 25 \\
\hline DC/CC & ko04510 & Focal adhesion & 0.001726 & 18 \\
\hline & ko04145 & Phagosome & 0.021728 & 14 \\
\hline & ko04070 & Phosphatidylinositol signaling system & 0.021728 & 10 \\
\hline & ko04530 & Tight junction & 0.025259 & 10 \\
\hline DC/F & ko05012 & Parkinson's disease & $3.92 \mathrm{E}-07$ & 14 \\
\hline & ko00190 & Oxidative phosphorylation & $1.50 \mathrm{E}-06$ & 14 \\
\hline & ko04066 & HIF-1 signaling pathway & 0.016405 & 5 \\
\hline
\end{tabular}




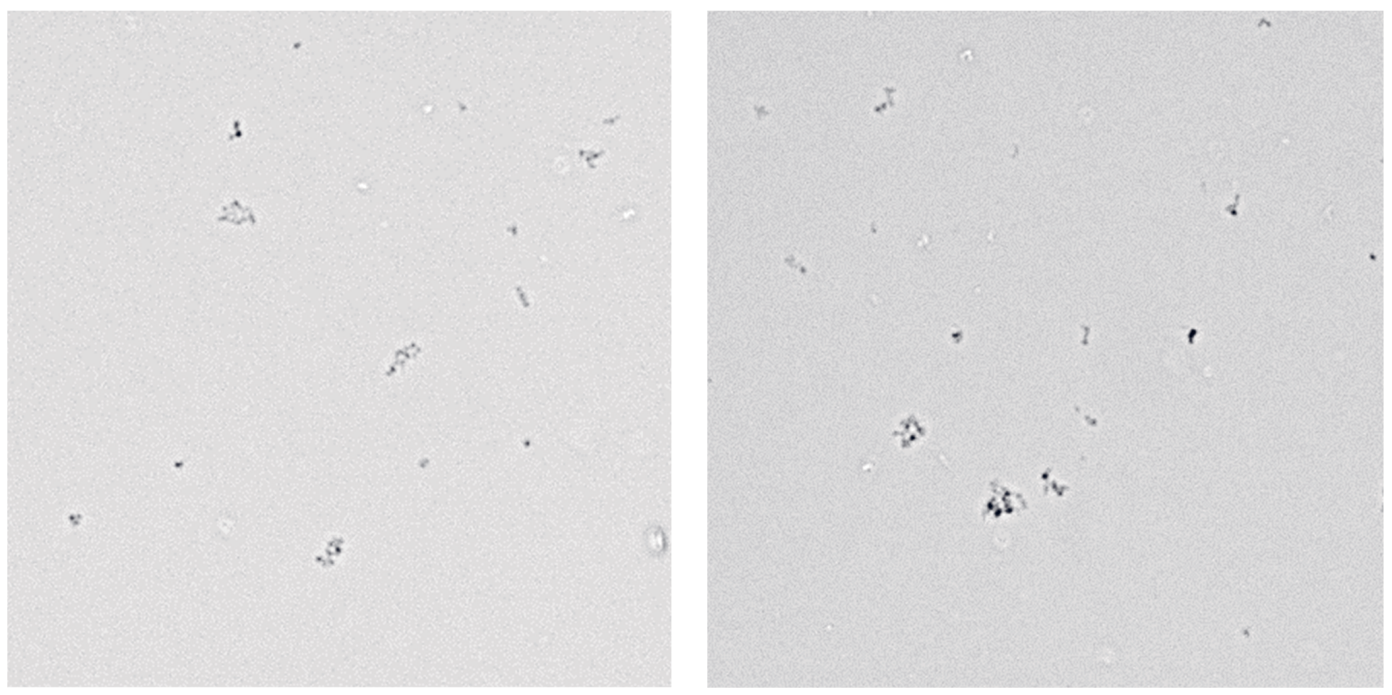

Figure S1. Micrographs (40X) of G. barretti cells cultured in OpM1 medium 


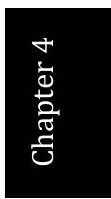


0 


\section{Chapter 5}

\section{CRISPR/Cpf1-mediated Gene Editing in Marine Sponge Geodia barretti}

This chapter is published as:

Hesp, K., Flores-Alvarez, J.L., Alexandru, A., et al. CRISPR/Cas12a-

mediated Genome Editing in Geodia barretti Sponge Cell Culture Front Mar Sci 7, 599825 (2020) 


\section{Abstract}

Sponges and sponge-derived microbes are the most prolific source of marine natural products, and many attempts have been made at creating a marine sponge cell line to produce such products. However, lacking insight in which nutrients sponge cells require to grow and poor genetic accessibility have hampered progress towards this goal. Recently, a new sponge-specific nutrient medium M1 has been shown to stimulate sponge cells to divide rapidly in vitro. In this study we demonstrate for the first time that sponge cells growing in M1 can be genetically modified using a CRISPR/Cas12a gene editing system. The 2',5'-oligoadenylate synthetase gene of cells from the boreal deepsea sponge Geodia barretti was disrupted, by inserting a short sequence of scrambled DNA using a single-stranded oligodeoxynucleotide donor template. Blue fluorescent marker gene TagBFP was inserted in an intron of the same gene and expressed by G. barretti cells. This shows that CRISPR/Cas12a can be used to disrupt and overexpress genes in sponge cells and represents an important step towards developing an optimized continuous sponge cell line to produce bioactive compounds. 


\subsection{Introduction}

Developing a marine sponge cell line to produce sponge-derived chemicals in vitro has been the holy grail of sponge biotechnology ever since the wide variety of bioactive secondary metabolites in marine sponges was discovered 9, 57, 60 . Many of these compounds have the potential to be developed into new drugs to combat cancer ${ }^{251,252}$, inflammatory disease ${ }^{253}$ and infections in humans ${ }^{36,41,112}$. Others could be used in industrial and commercial applications, for example as anti-fouling agents ${ }^{254}$, cosmetics and nutraceuticals ${ }^{255}$. Each of these applications potentially represents a multibillion-dollar market ${ }^{9}$. However, lack of sufficient biomass to produce compounds at the scale required for clinical trials or industrial application has been a major bottleneck ${ }^{32,252}$. Harvesting of wild sponges is neither economically nor environmentally sustainable and chemical synthesis of many sponge-derived chemicals is time-consuming and expensive due to their complex nature $\mathrm{e}^{9,32,252}$. Underlying metabolic pathways are often equally complex and poorly understood, making metabolically engineering recombinant yeasts or bacteria to produce compounds difficult ${ }^{9}$. Although mariculture can be suitable in some cases ${ }^{51}$, it does not allow to precisely control culture conditions or optimize productivity ${ }^{9}$. Culturing sponge cells in vitro has been proposed as a solution, with sponge cells producing the compound in a controlled environment that can be adapted and optimized. Furthermore, harvesting and downstream processing are simplified compared to mariculture.

Developing such sponge cell cultures has been challenging ${ }^{54,57,60}$. Little was known about the nutrients sponge cells need to proliferate, resulting in shortlived primary cultures ${ }^{57}$. Cells in such cultures quickly became less viable and metabolically active and were often overgrown by bacteria or fung ${ }^{15}$. Recently, it was shown that cells of the Caribbean sponge Dysidea etheria were more metabolically active in sponge-specific nutrient medium M1, which contains optimized amino acid concentrations ${ }^{103}$. Furthermore, cells of various other sponge species divided rapidly in $\mathrm{M}^{136}$. Cells of 3 species from the genus Geodia, one of which was Geodia barretti,, responded strongly to M1 with limited variation between individuals. Cells could be subcultured 3-5 times and reached 
a maximum of 7 population doublings ${ }^{136}$. Having proliferating cells is an important step towards large-scale production of bioactive compounds in bioreactors. Moreover, it is required to develop molecular tools that will be needed to create and optimize production strains ${ }^{256}$.

One of the most well-known genetic engineering tools is CRISPR/Cas gene editing. CRISPR (clustered regularly-interspaced palindromic repeat) arrays were discovered as adaptive immune systems in prokaryotes. Short sequences of foreign DNA incorporated into CRISPR arrays in the genome are transcribed and processed into CRISPR RNAs (crRNA), that guide endonucleases to cleave the complementary DNA sequence ${ }^{257}$. These systems use a protospacer-adjacent motif (PAM) to distinguish between foreign DNA and the host genome, since the PAM is present in the foreign DNA but not in the CRISPR array. By altering the crRNA sequence, CRISPR/Cas systems can be used to specifically target any DNA sequence that contains the PAM (Figure 1). The endonuclease induces a doublestranded break (DSB) that can be repaired in two ways: the first, nonhomologous end joining (NHEJ), often leads to small insertions/deletions causing frameshifts, while the second, homology-directed repair (HDR), can be used to insert DNA sequences by providing a repair template with homology up- and downstream of the target site ${ }^{258}$ (Figure 2A). CRISPR/Cas9 is the most commonly used CRISPR system for gene editing but requires a trans-encoded small crRNA (tracrRNA), either separately or connected to the crRNA for convenience ${ }^{259}$. Another endonuclease, called Cas12a or Cpf1, was discovered more recently and is an attractive alternative to Cas9, because it does not require a tracrRNA, and instead uses 1 short guide RNA (gRNA) molecule (Figure 1) ${ }^{260,261}$. CRISPR/Cas12a has been used to edit genomes in various cell cultures of plants, animals and humans ${ }^{262}$. To test whether the the highly versatile CRISPR/Cas12a system could also edit the genome of marine sponge cells in vitro, we designed an experiment to introduce DSBs in the genome of $G$. barretti cells and incorporate either a short insert (108 base pair (bp)) (Figure 2A) or a longer construct carrying blue fluorescent marker gene TagBFP (Subach et al., 2008) through HDR (Figure 2C). 


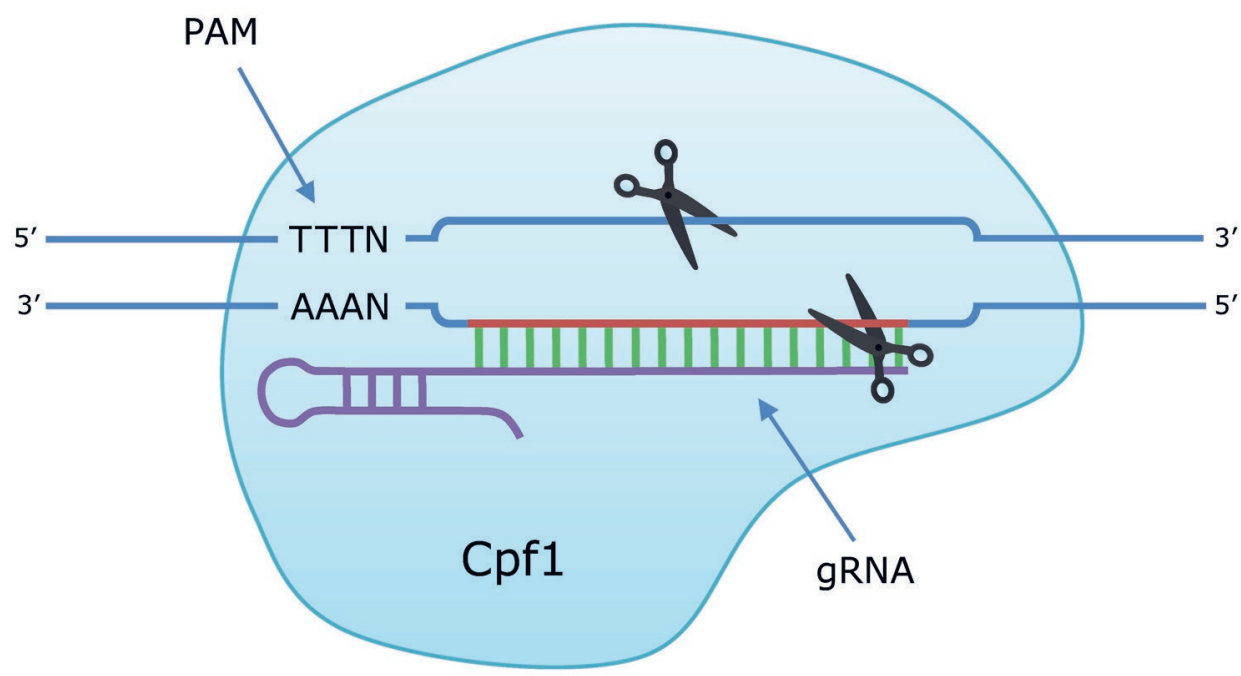

Figure 1. Introduction of a double stranded break (DSB) in DNA by CRISPR/Cas12a (Cpf1). The guide RNA (gRNA) directs the Cas12a endonuclease to the homologous target sequence. When the protospacer-adjacent motif (PAM) is recognized and the crRNA hybridizes with the target sequence, the nuclease domains of Cas $12 a$ introduce breaks in each strand, 19 nt (target strand) and 23 nt (complementary strand) downstream from the PAM sequence.

Since the full genome of $G$. barretti is not yet available, the 2',5'-oligoadenylate synthetase 1 (OAS1Ab) gene (Acc. HQ644329) was targeted, of which a partial genomic DNA sequence was reported ${ }^{263}$. Expressing heterologous genes on plasmids in sponge cells has been a challenge in the past ${ }^{59}$. Therefore, preassembled ribonucleoprotein complexes (RNPs) of recombinant Cas12a protein and the guide RNA (gRNA) were used, rather than a plasmid encoding Cas12a and the gRNA. This method has been used to edit genes in mammalian cells ${ }^{264}$, 265, zebrafish ${ }^{41}$, mouse embryos ${ }^{264}$, and plants ${ }^{266}$. We confirmed that the scrambled DNA sequence was inserted by amplifying the region with polymerase chain reaction (PCR) (Figure $2 \mathrm{~B}$ ) and Sanger sequencing. Fluorescence microscopy was used to verify that the TagBFP gene was inserted and expressed. 
A

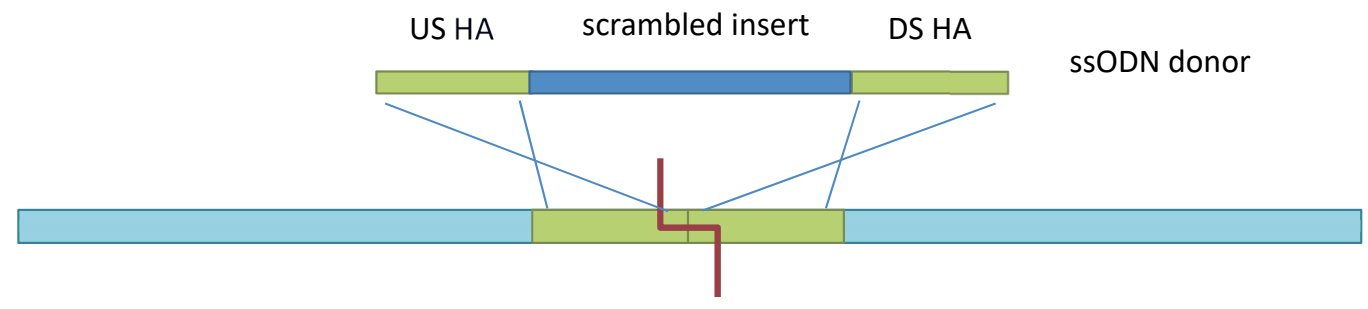

DSB

B

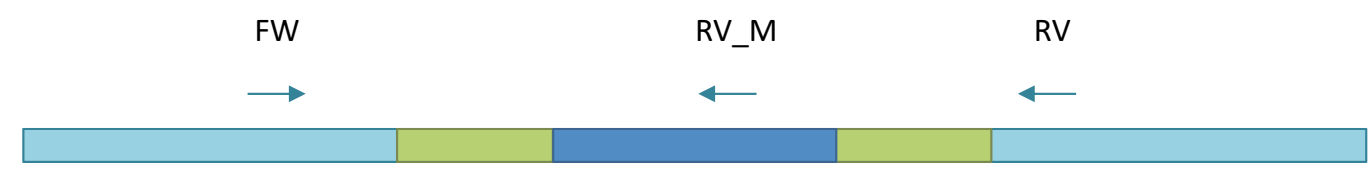

C

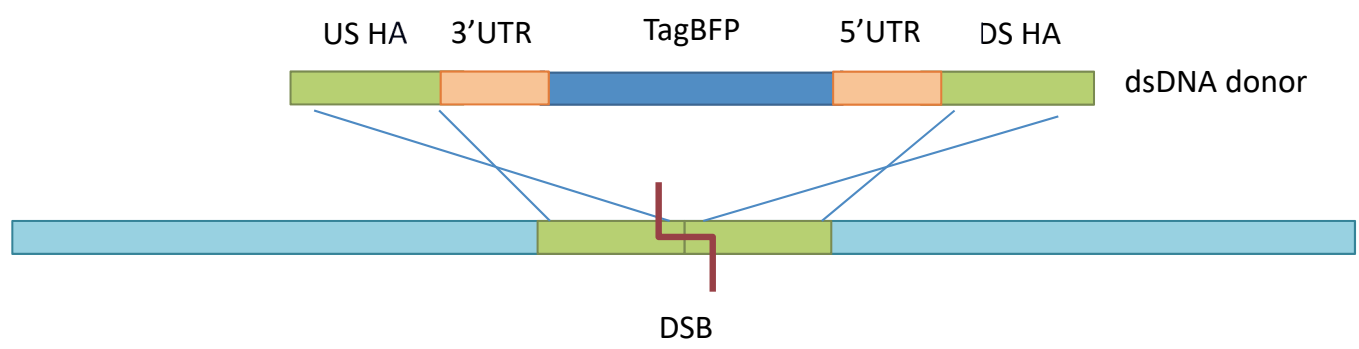

D

3'UTR TagBFP 5'UTR

Figure 2. Experimental design. A. After Cas12a introduces the DSB at the target site, HDR takes place between the US HA and DS HA of the SSODN donor template and the homologous regions US and DS from the DSB. B. The insert is incorporated between the US and DS homologous region. Two PCR reactions determine whether mutation was successful: 1. Reverse primer anneals inside of the insert (RV_M), yielding a product of 910 $b p$, providing HDR took place. 2. Reverse primer anneals to the wild type gene sequence (RV), yielding a $1025 \mathrm{bp}$ (wild type) or 1127 (mutant) product. C. As with the ssODN donor, the US and DS HA recombine with their homologous regions US and DS of the DSB made by Cas12a. D. The TagBFP gene with 5' and 3' UTRs of the S. domuncula actin locus is inserted into the genome in reverse orientation. 


\subsection{Materials \& Methods}

\subsubsection{Sampling, dissociation \& cryopreservation}

The $G$. barretti individual used in this study was collected with a 500-meter-deep trawl in the Norwegian fjords $\left(59^{\circ} 58.8^{\prime \prime} \mathrm{N} 5^{\circ} 22.4^{\prime \prime} \mathrm{E}\right)$. Sponge cells were dissociated by squeezing small pieces of tissue through a sterile gauze into filtered sea water (FSW). The cells were passed through a $40 \mu \mathrm{m}$ cell strainer (Falcon ${ }^{\circledR}$ ), then centrifuged ( $300 \times g, 5$ minutes) and resuspended in ASW twice. The cells were diluted 100x in FSW and counted using a disposable hemocytometer (C-chip ${ }^{\mathrm{TM}}$, Neubauer improved). Cells were centrifuged once more (300 x g, 5 minutes) and resuspended in cryoprotectant ( $10 \%$ fetal bovine serum (FBS) and 10\% dimethyl sulfoxide (DMSO) in FSW) 54, 86, 102 at approximately $1.00 \mathrm{E}+08$ cells $/ \mathrm{mL}$. Aliquots $(1 \mathrm{~mL})$ of this cell suspension in cryoprotectant, in cryogenic vials (Fisherbrand), were cooled at $1^{\circ} \mathrm{C}$ per minute to $-80^{\circ} \mathrm{C}$ in a Nalgene ${ }^{\circledR} \mathrm{Mr}$. Frosty freezing container.

\subsubsection{Medium preparation}

Artificial seawater (ASW) (modified from Zhang et al. (2004) was prepared by dissolving the powdered ingredients in demineralized water $\left(\mathrm{dH}_{2} \mathrm{O}\right)$ at the following concentrations: $23.30 \mathrm{~g} / \mathrm{L} \mathrm{NaCl}, 10.20 \mathrm{~g} / \mathrm{L} \mathrm{MgCl}_{2}, 4.02 \mathrm{~g} / \mathrm{L}$ Trizma $\mathrm{HCl}$, $2.97 \mathrm{~g} / \mathrm{L}$ Trizma Base, $1.1 \mathrm{~g} / \mathrm{L} \mathrm{CaCl}_{2}$ and $1 \mathrm{~g} / \mathrm{L} \mathrm{KCl}{ }^{97}, 102,103$. The solution was sterilized by filtering through a $0.22 \mu \mathrm{m}$ mesh. M1 medium was prepared following the original protocol ${ }^{103}$. Medium 199 powder (Sigma Aldrich, M3769) was dissolved in $\mathrm{dH}_{2} \mathrm{O}$, then salts were added at concentrations like those in seawater, leading to a salinity of $33.5 \mathrm{ppt}$ in M1, approximating the salinity of 35 ppt in seawater. The $\mathrm{pH}$ of the M1 medium (7.9) was also like that of seawater (8.1). Amino acids were added (Table S2) and finally, rifampicin (Sigma Aldrich, R3501) and amphotericin B (Sigma Aldrich, A2411) to control contamination by bacteria and fungi, respectively.

\subsubsection{Cell culture}

Before cultures were inoculated, cryopreserved cells were thawed quickly in a water bath set at $50{ }^{\circ} \mathrm{C}$ to prevent cells being damaged by ice crystals. The cells 
were washed twice with artificial sea water (ASW) by centrifuging at $300 \times \mathrm{g}$ for 5 minutes, removing the supernatant and resuspending them in $1 \mathrm{~mL}$ ASW. Next, cells were counted microscopically using disposable hemocytometers (C-chip ${ }^{\mathrm{TM}}$, Neubauer improved) to determine the cell concentration. M1 medium was inoculated at $3.0 \mathrm{E}+06$ cells $/ \mathrm{mL}$ in 12 well plates with $1 \mathrm{~mL}$ cell suspension per well. To passage the cells, cell concentrations were determined, and the cell suspension was diluted back to $3.0 \mathrm{E}+06$ cells $/ \mathrm{mL}$ with fresh $\mathrm{M} 1$ medium.

\subsection{4 gRNA design \& in vitro assay}

The online tool CRISPOR ${ }^{\circledR}$ (v4.97) was used to design 3 gRNAs targeting the $3^{\text {rd }}$ exon and 2 gRNAs targeting the $2^{\text {nd }}$ intron (Table S1) in the genomic G. barretti OAS1Ab sequence (Acc. No. HQ644329.1) and ordered as synthetic Cas12a gRNA oligonucleotides at Integrated DNA Technologies (Leuven, Belgium). The recombinant Cas12a enzyme (LbCas12a, derived from Lachnospiraceae bacterium ND2006) contains a poly-histidine tag and was overexpressed in E. coli and purified using immobilized metal affinity chromatography (IMAC) (Loughran \& Walls, 2011) by Mihris Naduthodi from the Microbiology department of Wageningen University \& Research. All 3 crRNAs were reconstituted with nuclease free water (NFW) to a concentration of $300 \mathrm{nM}$ and then tested in vitro for their efficiency in cleaving a 1025 bp PCR product amplified from G. barretti genomic DNA (gDNA) (see 'Genomic DNA extraction \& PCR' section for DNA extraction, PCR conditions and primers (RV-WT)) in complex with the Cas12a enzyme. The protocol 'In vitro digestion of DNA with EnGen ${ }^{\circledR}$ Lba Cas12a (Cas12a)' (New England BioLabs, M0653) was followed. For each crRNA, $3 \mu$ of $300 \mathrm{nM}$ stock solution (final concentration $30 \mathrm{nM}$ ) was mixed with $1 \mu \mathrm{l}$ of $1 \mu \mathrm{M}$ stock of Cas12a enzyme (final concentration $33.3 \mathrm{nM}$ ) to allow formation of the ribonucleoprotein (RNP) complexes. The mixture was incubated for 10 minutes at $37^{\circ} \mathrm{C}$ in $3 \mu \mathrm{l} 10 x$ NEBuffer 2.1 Reaction Buffer $(500 \mathrm{mM} \mathrm{NaCl}, 100 \mathrm{mM}$ Tris- $\mathrm{HCl}$, $10 \mathrm{mM} \mathrm{MgCl}, 100 \mu \mathrm{g} / \mathrm{mL}$ bovine serum albumin (BSA), pH 7.9) diluted with 20 $\mu \mathrm{l} \mathrm{NFW}$. Subsequently $3 \mu \mathrm{l}$ of $30 \mathrm{nM}$ DNA fragment stock solution (final concentration $3 \mathrm{nM}$ ) was added to the reaction mixture and incubated with the RNPs for 10 minutes at $37^{\circ} \mathrm{C}$. The final reaction volume after adding the PCR product was $30 \mu$ l. Samples were incubated for 10 minutes with Proteinase K (20 
$\mathrm{ng} / \mu \mathrm{l})$ to stop the reaction. Finally, $10 \mu \mathrm{L}$ of the reaction mixture was run on a $2 \%$ agarose gel at $135 \mathrm{~V}$ for 50 minutes.

\subsection{5 ssODN and dsDNA donor template design}

Homology-directed repair of DSBs requires a donor template with homology arms up- and downstream of the target site. Synthetic ssODNs were ordered from Integrated DNA Technologies, designed featuring 30-40 bp homology arms flanking a $108 \mathrm{bp}$ scrambled DNA insert containing stop codons in all 3 reading frames (Table S1). A plasmid containing the dsDNA donor template, consisting of the TagBFP gene with $S$. domuncula actin 5' and 3' UTRs in reverse orientation, flanked by the US (533 bp) and DS (601 bp) HA, was ordered from Integrated DNA Technologies (Figure S1).

\subsubsection{Lipofection}

Cells of 1 individual of $G$. barretti were cultured in 12-well plates with $1 \mathrm{~mL}$ cell suspension/well, starting at a density of $3.00 \mathrm{E}+06$ cells $/ \mathrm{mL}$ in $\mathrm{M} 1$ medium and a temperature of $4{ }^{\circ} \mathrm{C}^{136}$ Hesp et al., in prep, Chapter 3 of this thesis). The ssODNs were transfected together with the RNPs using Lipofectamine ${ }^{\mathrm{TM}} \mathrm{CRISPRMAX}^{\mathrm{TM}}$ (Thermo Fisher Scientific, CMAX00008), since lipofection has been used successfully to transfect sponge cells in vitro ${ }^{59}$. The TagBFP repair construct plasmid was introduced using Lipofectamine ${ }^{\mathrm{TM}} 3000$ (ThermoFisher Scientific, L3000015), 10 minutes before the RNPs were added using Lipofectamine ${ }^{\mathrm{TM}}$ CRISPRMAX ${ }^{\mathrm{TM}}$. For each sample, $0.5 \mu \mathrm{g}$ of plasmid DNA and $5 \mu \mathrm{L}$ of $\mathrm{P} 3000^{\mathrm{TM}}$ reagent were added to $50 \mu \mathrm{L}$ Opti-MEM ${ }^{\mathrm{TM}}$ I Reduced Serum Medium (Gibco, 31985062 ) in a $1.5 \mathrm{~mL}$ Eppendorf tube, and $3 \mu \mathrm{L}$ Lipofectamine $^{\mathrm{TM}} 3000$ in $50 \mu \mathrm{L}$ Opti-MEM $^{\mathrm{TM}}$ in a second tube. The contents of both tubes were mixed and incubated for 15 minutes at room temperature before the mixture was added dropwise to the sponge cells. RNPs were formed in vitro by preparing the following for each sample: $0.5 \mu \mathrm{g}$ of gRNA E32 or 122, $2.5 \mu \mathrm{g}$ of Cas12a, and $5 \mu \mathrm{l}$ of Cas9 Plus ${ }^{\mathrm{TM}}$ Reagent in $50 \mu \mathrm{l}$ Opti-MEM ${ }^{\mathrm{TM}}$ in 1 Eppendorf tube, and in another tube $3 \mu \mathrm{l}$ of Lipofectamine ${ }^{\circledR}$ CRISPRMAX ${ }^{\mathrm{TM}}$ transfection reagent in $50 \mu \mathrm{l}$ Opti$\mathrm{MEM}^{\mathrm{TM}}$. The contents of both tubes were combined and incubated for 10 minutes at room temperature. For transfection with the $108 \mathrm{bp}$ scrambled DNA 
construct, $0.5 \mu \mathrm{g}$ sSODN was mixed in at this point, and the solution was then added dropwise to the cells. For TagBFP plasmid transfection, no DNA was added at this stage.

\subsubsection{Genomic DNA extraction \& PCR}

After 4 days of incubation, gDNA was obtained from transfected and nontransfected cells using the High Pure PCR Template Preparation Kit from Roche Life Science. Two PCR reactions were used to detect whether the G. barretti genome was edited successfully (Figure $2 \mathrm{C}$ ). Both reactions used the same forward primer (FW 5'-ATGGCTAGCCCAGGACTTAGG), while the reverse primer bound either downstream of the target site (RV-WT 5'-AACAATGTGGTGCACTCGAA) or inside the scrambled DNA insert (RV-M 5'-CTATCCCCACCCCCACATTC). The first reaction should always yield a product, either of $1025 \mathrm{bp}$ in the wild type, or $1127 \mathrm{bp}$ in case of mutated cells. The second reaction would only amplify a $910 \mathrm{bp}$ product if some cells in the culture were successfully mutated. The PCR mixture consisted of $25 \mu \mathrm{l} 2 \mathrm{x}$ Phusion HighFidelity PCR Master Mix with HF Buffer (Thermo Scientific ${ }^{\top M}$, F531S), $2.5 \mu \mathrm{l} 10$ $\mu \mathrm{M}$ working solution of both primers and $\sim 20 \mathrm{ng}$ of gDNA brought to a total reaction volume of $50 \mu \mathrm{l}$ with MilliQ (MQ) water. PCR conditions were as follows: 15 seconds initial denaturation at $98{ }^{\circ} \mathrm{C}$ followed by 35 cycles of $10 \mathrm{~s}$ denaturation at $98^{\circ} \mathrm{C}, 30 \mathrm{~s}$ primer annealing at $59^{\circ} \mathrm{C}$ and $45 \mathrm{~s}$ extension at $72^{\circ} \mathrm{C}$ and then $10 \mathrm{~min}$ final extension at $72^{\circ} \mathrm{C}$. Amplified products were purified using the DNA Clean \& Concentrator-5 (capped) kit from Zymo Research (D4014) and eluted in a final volume of $10 \mu \mathrm{MQ}$ water. Product concentration in the purified samples was measured using NanoDrop ${ }^{\mathrm{TM}}$ One (Thermo Scientific ${ }^{\mathrm{TM}}$ ). Approximately $1 \mu \mathrm{g}$ of purified PCR product per sample in a total volume of $6 \mu \mathrm{l}$ with $1 \mu \mathrm{l} 6 \mathrm{x}$ loading dye (Thermo Scientific ${ }^{\mathrm{TM}}$, R0611) was run on a $1 \%$ agarose gel at $70 \mathrm{~V}$ for 35 minutes, $500 \mathrm{ng}$ GeneRuler $1 \mathrm{~kb}$ DNA Ladder (Thermo Scientific ${ }^{\top M}, \mathrm{SM} 0311$ ) in a total volume of $3 \mu \mathrm{l}$ with $0.5 \mu \mathrm{l} 6 \mathrm{x}$ loading dye was used as a marker on either side of the samples. 


\subsubsection{Mutation analysis}

To verify that the $108 \mathrm{bp}$ scrambled DNA sequence was successfully inserted into the $3^{\text {rd }}$ exon of the OAS1Ab gene, $15 \mathrm{ng}$ of purified PCR product per $100 \mathrm{bp}$ length ( $140 \mathrm{ng}$ for WT, $155 \mathrm{ng}$ for M products) for each sample was sent to BaseClear for Sanger sequencing as Long run (up to $1100 \mathrm{nt}$ ) Quick Shot Premix samples in a total volume of $20 \mu \mathrm{l}$ with $25 \mathrm{pmol}$ primer. Sequences were aligned using SnapGene ${ }^{\circledR}$ (v4.3) and to compare sequences obtained through Sanger sequencing to the genomic DNA sequence of the G. barretti OAS1Ab gene (Acc. No. HQ644329.1). Whether TagBFP was successfully inserted into the $2^{\text {nd }}$ intron of the OAS1Ab gene and expressed by the cells was determined by fluorescence microscopy, using the DAPI filter on an EVOS FL Auto Cell Imaging System ${ }^{\mathrm{TM}}$.

\subsection{Results}

We designed a single-stranded oligodeoxynucleotide (sSODN) construct with short homology arms (HA) (30-40 bp) to demonstrate that CRISPR/Cas12a RNPs could be introduced into and edit the genome of $G$. barretti cells. Donor templates provided as SSODN mediate HDR more efficiently than their doublestranded (dsDNA) counterparts ${ }^{267,268}$ and shorter ssODN constructs are more efficient than longer ssODNs ${ }^{269}$. The HAs matched regions upstream (US) and downstream (DS) of the target site in the $3^{\text {rd }}$ OAS1Ab exon and flanked a $108 \mathrm{bp}$ scrambled DNA insert (Figure 2A), containing stop codons in all 3 reading frames. To test whether the CRISPR/Cas12a system could be used to overexpress a gene, we designed a construct containing fluorescent marker gene TagBFP, flanked by the $5^{\prime}$ untranslated region (UTR) and 3' UTR of the Suberites domuncula actin locus ${ }^{270}$ (Figure $2 \mathrm{C}$ ). Since no intergenic sequences were available, the TagBFP gene would be inserted in the $2^{\text {nd }} O A S 1 A b$ intron in reverse orientation, to reduce interfering of the marker gene with the function of the OAS1Ab gene. The approximately 2 kilobase $(\mathrm{kb})$ dsDNA construct required longer HAs of $0.5-1 \mathrm{~kb}$ each $^{271,272}$ and our design therefore featured an US HA of 533 bp and a DS HA of $601 \mathrm{bp}$. 
A

FW (1 .. 21)

$\mathrm{RV}(1006 . .1025)$

\begin{tabular}{|c|c|c|c|c|}
\hline & $250^{\prime}$ & $500^{1}$ & $750^{1}$ & $1000^{1}$ \\
\hline Exon 1 & Exon 2 & 121122 & & \\
\hline
\end{tabular}

B

E33

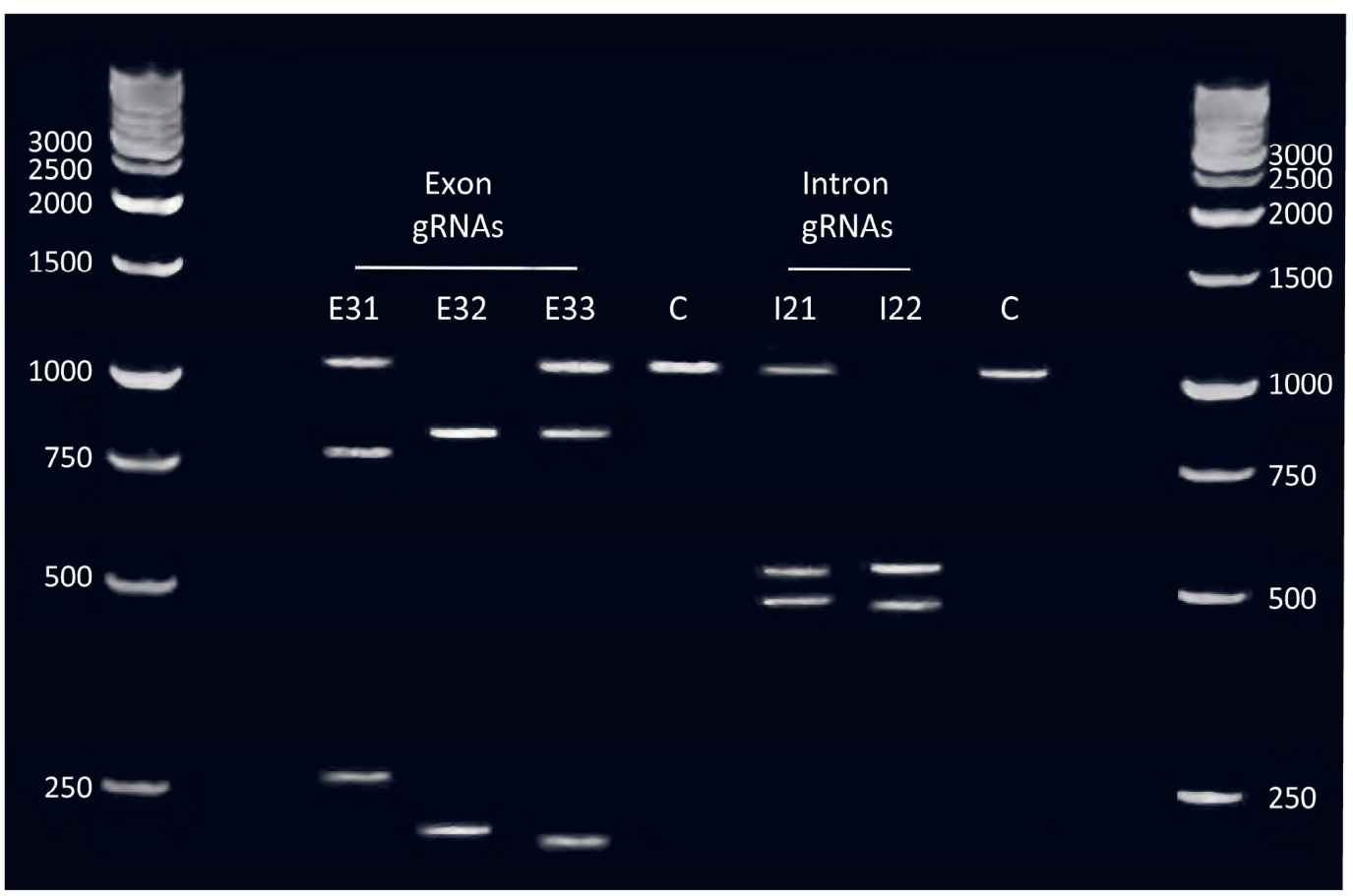

Figure 3. A. Locations of gRNAs on the OAS1Ab wild-type PCR product of $1025 \mathrm{bp}$. B. In vitro assay testing how efficiently 5 gRNAs can direct Cas12a to cleave their respective target sites in the 1025 bp wild-type PCR product. Of the $3 \mathrm{gRNAs}$ targeting exon 3 (left), only gRNA E32 fully degraded the DNA, while gRNA 122 targeting intron 2 also degraded all DNA. gRNA E32 and I22 were selected to edit the G. barretti OAS1Ab gene. 


\subsection{1 gRNA design \& in vitro assay}

Synthetic gRNAs were designed for both target sites in the G. barretti OAS1Ab gene, 3 gRNAs targeted the $3^{\text {rd }}$ exon and 2 gRNAs targeted the $2^{\text {nd }}$ intron, as only 2 suitable target sequences for Cas12a were present in the intron that would leave space for $>0.5 \mathrm{~kb}$ US and DS HAs (Figure $3 \mathrm{~A}$ ). An in vitro assay in which RNPs of Cas12a with each of the 5 gRNAs were added to a PCR product of the wild-type OAS1Ab gene was used to test how efficiently each gRNA could induce a DSB (Figure 2B, FW + RV primers). The same PCR product incubated with Cas12a but without a gRNA was used as a negative control (C). All 5 tested gRNAs induced cuts at their target sites (Figure 3B), but only exon 3 gRNA 2 (gRNA E32), and intron 2 gRNA 2 (gRNA 122) degraded all DNA present. We selected gRNA $\mathrm{E} 32$ and 122 to edit the $G$. barretti genome.

\subsubsection{Knock-out OAS1Ab}

G. barretti cells were transfected with RNPs of Cas12a and gRNA E32 accompanied by the ssODN donor template to insert the 108 bp scrambled DNA sequence with stop codons in all 3 reading frames, leading to truncated OAS1Ab proteins. Whether transfected cells were successfully mutated was checked using 2 PCR reactions (Figure $2 \mathrm{~B}$ ). The $1^{\text {st }}$ reaction should always yield an either 1025 bp (wild type, W) or 1127 bp (mutant, M) product or both, while the $2^{\text {nd }}$ reaction only amplified a $910 \mathrm{bp}$ product if mutated cells were present in the culture. Both the $M$ and $W$ PCR reactions amplified their respective products in 2 out of $3(T 1+T 2)$ transfected cell populations (Figure 4), suggesting the $108 \mathrm{bp}$ scrambled DNA sequence was inserted into the OAS1Ab gene.

No separate $1025 \mathrm{bp}(\mathrm{W})$ and $1127 \mathrm{bp}(\mathrm{W})$ products were detected in T1 and T2, suggesting that the bands were either too close together, or $M$ genes were present in small numbers and therefore not amplified due to the majority of $\mathrm{W}$ genes in the extracted gDNA. The $3^{\text {rd }}$ transfection (T3) yielded the same results as the negative control of cells transfected with Cas12a and ssODN donor, but without the gRNA (C), where only the W PCR amplified its 1025 bp product (Figure $4 A$ ). Sanger sequencing of the M PCR product returned the expected sequence (Figure $4 B$ ), confirming the OAS1Ab gene was successfully edited. 


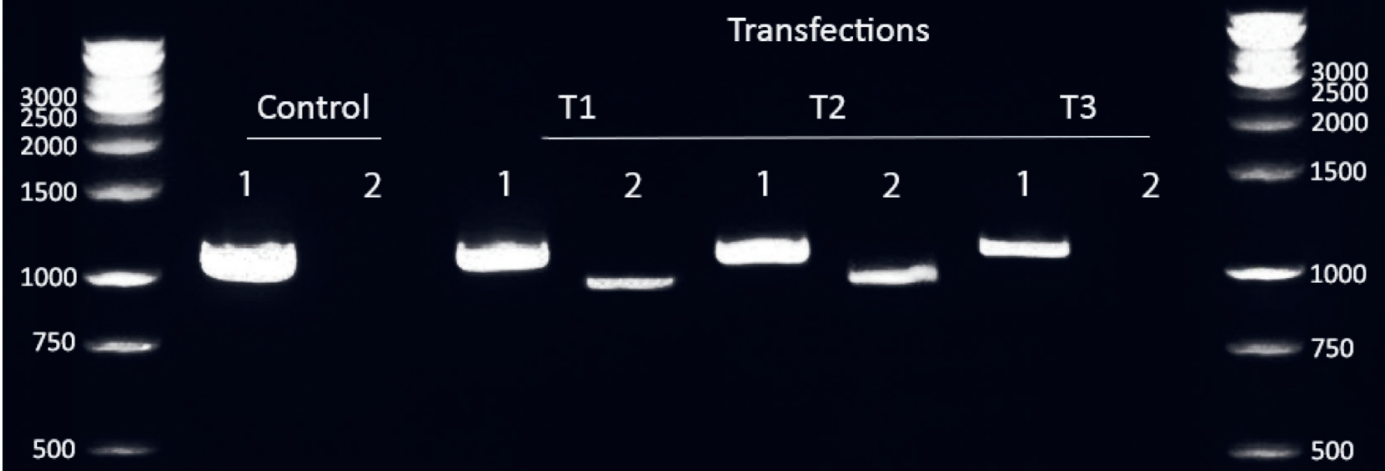

B

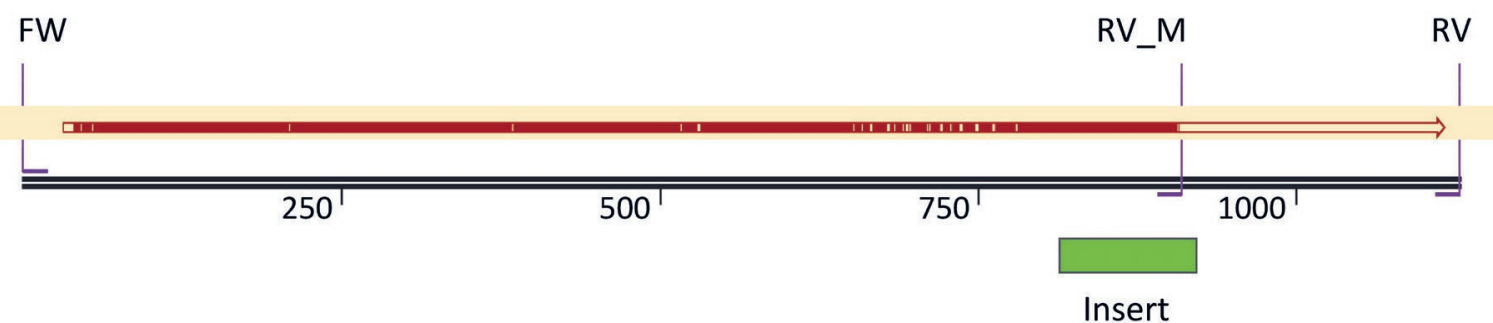

Figure 4. $P C R$ and sequencing results confirm that the scrambled DNA sequence was inserted in populations of transfected cells. A. Results of 2 PCR reactions amplifying part of the OAS1Ab gene in DNA extracted from 3 transfected G. barretti cell populations (T1, 2 and 3). Both reactions used the same FW primer. PCR 1 used the wildtype RV primer and should always amplify a 1,025 bp (W) or 1,127 bp (M) product. PCR 2 contained the mutant RV (RV_M) primer and only yields a 910 bp product $(M)$ if the gene was successfully edited. The control (C) contained DNA extracted from cells that were treated the same as T1-3, except that no gRNA was provided in the transfection. T1 and T2 both yielded the M product, indicating the $108 \mathrm{bp}$ scrambled DNA sequence was inserted at the target site in some cells. B. Alignment of the sequencing results of the $910 \mathrm{bp}$ mutant product with the sequence expected if the OAS1Ab gene was successfully edited. Red indicates matching sequences, while yellow indicates gaps. Since the sequenced PCR product was obtained using PCR 2, the sequence between RV_M and RV was not present in the sequence read. 


\subsubsection{Expression TagBFP marker gene}

To insert and overexpress the blue fluorescent TagBFP gene, G. barretti cells were first transfected with the plasmid containing the TagBFP repair construct (Figure $2 \mathrm{C}$ ), then after 10 minutes transfected again with the RNPs of Cas12a and gRNA 122. Whether cells were expressing TagBFP was determined by fluorescence microscopy. Sporadic blue fluorescence was observed in cells transfected with RNPs and the TagBFP repair construct (Figure 6). No fluorescent sponge cells were observed in controls transfected with Cas12a but without the gRNA. The low number of mutants combined with difficulties extracting DNA made obtaining clear PCR results not possible.

\subsection{Discussion}

We previously reported that cells from several species of marine sponges divided rapidly in M1 medium and could be subcultured for several weeks ${ }^{136}$. Now that proliferating cells are available for multiple species, the next step is to improve various characteristics of the cells to create efficient production strains for sponge-derived biopharmaceuticals, like increasing how many population doublings cells can reach to produce more biomass, activating or optimizing pathways that synthesize potential drug candidates, or improving energy efficiency. Molecular tools are necessary to improve these and other traits. CRISPR/Cas systems are currently the most prominent and promising tools, with new applications being reported in rapid succession ${ }^{273-276}$.
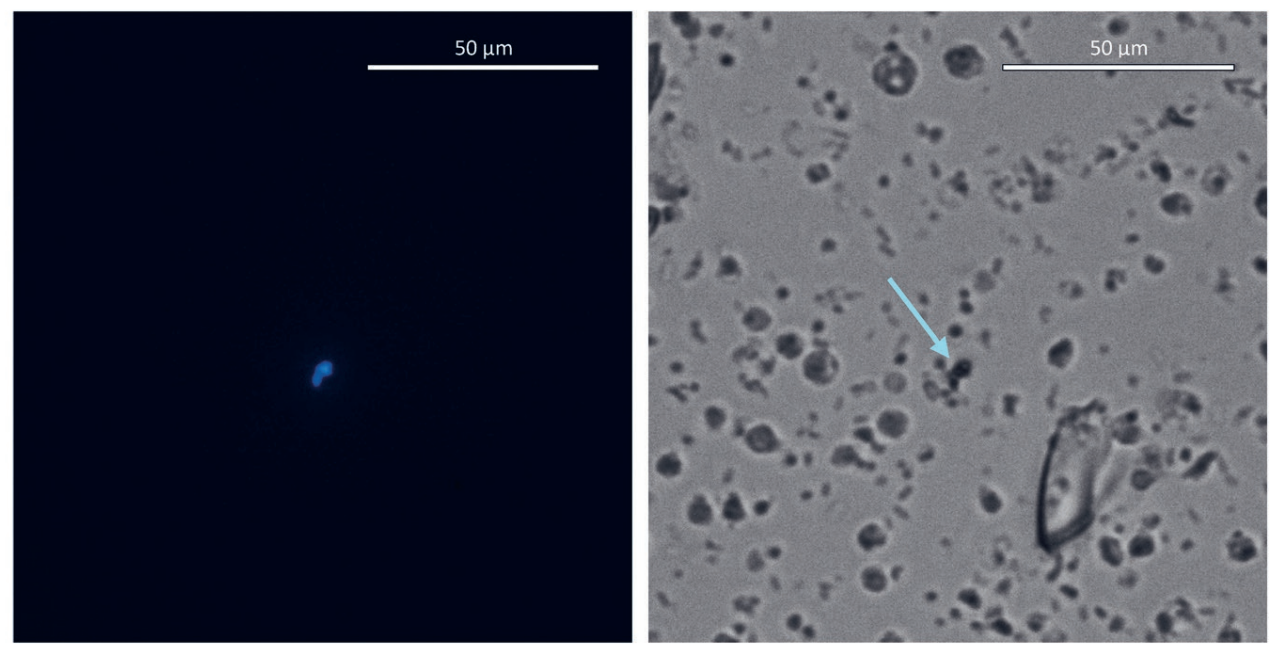

Figure 5. Blue fluorescing cells in images taken with DAPI (left) and transmitted light (right) filters at 80x magnification. Arrow indicates the cells that fluoresce blue in the DAPI filter image. 
Here we report the first successful use of a CRISPR/Cas system in marine sponges. We demonstrate that CRISPR/Cas12a (Cpf1) can be used to target and edit sites in the G. barretti genome, and sequences can be inserted at such sites using HDR. We disrupted the G. barretti OAS1Ab gene by inserting a $108 \mathrm{bp}$ scrambled DNA sequence with stop codons in all 3 reading frames, ensuring no functional protein could be synthesized. In humans, transcription of OAS genes is induced by interferon activity ${ }^{277}$ to defend against viruses ${ }^{278}$ as well as induce apoptosis in tumor cells ${ }^{279}$. Low OAS1 expression levels in prostate cancer predicted a poor prognosis ${ }^{280}$, and its overexpression led to apoptosis or G1 arrest in human colorectal cancer cells, associated with higher expression levels of p53, BAX and p21 ${ }^{281}$. What role OAS1Ab has in sponges has not yet been determined ${ }^{263}$, but examining how disrupting this gene affects sponge cells in culture would shed light on this question. If this role is similar in sponges as in humans, disrupting this gene could help immortalize sponge cells. As more sequence data become available, the method presented here could be used to disrupt other targets, like well-known tumor-suppressor genes, but also metabolic genes to direct energy and carbon sources towards producing compounds of interest, or unknown genes to dissect their functions.

We transfected cells with reporter constructs encoding TagBFP flanked by the promoter and terminator regions of the $S$. domuncula actin locus ${ }^{270}$. TagBFPtransfected cells did sporadically display bright blue fluorescence that was not observed in controls, but the efficiency was extremely low. More efficient gene editing in $G$. barretti could be achieved by optimizing the lipofection protocol or increasing how efficient cells can perform HDR with the donor template. Varying the length of the homology arms can greatly influence HDR efficiency ${ }^{282,283}$, and recently a new promising method was developed, where the dsDNA template is provided on a plasmid, flanked by target sites for the same gRNA as will be used to edit the genome ${ }^{284,285}$. Another option would be to select for cells expressing a marker gene that protects them from an otherwise lethal chemical such as geneticin (G418) or hygromycin B ${ }^{286}$. Once larger constructs can be inserted more efficiently, this technique could be used to overexpress oncogenes or genes in pathways that synthesize potential biopharmaceuticals to increase productivity. 


\subsection{Conclusion}

Our results represent the first venture into genetically modifying marine sponge cells. It has now become possible to accurately disrupt specific genes to study and alter how genes function in these organisms. More research is needed to further optimize HDR in sponge cells to efficiently express genes. CRISPR/Cas systems are versatile and have been used in many types of organisms, now including $G$. barretti, which bodes well for their use in other sponge species. However, because sponge species and individuals often respond differently to certain treatments ${ }^{136}$, it is likely that methods will need to be optimized on a case-by-case basis. Nevertheless, we have gained a powerful tool and taken another step towards creating and improving sponge cell strains to produce sponge-derived pharmaceuticals in quantities that allow clinical trials and ultimately, treat disease in patients with novel, sponge-derived drugs. 


\subsection{Supplementary material}

Table S1. Design gRNAs and ssODN donor template. Target sequences and PAM sequence of gRNAs designed for the $3^{\text {rd }}$ exon (E31, E32 and E33) and the $2^{\text {nd }}$ intron (I21 and 122). Homology arms in the ssODN donor template for target E32 are shown, as well as sequence of the scrambled DNA insert (stop codons in red).

\begin{tabular}{|c|c|c|c|c|c|}
\hline \multicolumn{3}{|c|}{ Target site } & \multicolumn{2}{|c|}{ Homology arms } & Insert \\
\hline gRNA & PAM & Sequence & Upstream & Downstream & Sequence \\
\hline E31 & TTTC & $\begin{array}{c}\text { TCTTCAGGA } \\
\text { AAATTTTCC } \\
\text { AAGCT }\end{array}$ & & & $\begin{array}{l}\text { TAGGGTAGTATAGTG } \\
\text { TAAGATAAGTAATTA }\end{array}$ \\
\hline E32 & TTTA & $\begin{array}{c}\text { GTGTATTTG } \\
\text { ACATCATTA } \\
\text { AGGCT }\end{array}$ & $\begin{array}{l}\text { AAATTGGTTTT } \\
\text { AGTGTATTTGA } \\
\text { CATCATTA }\end{array}$ & $\begin{array}{c}\text { GCTCCCTGGGGC } \\
\text { ATGGAACTTCTGT } \\
\text { TGAGG }\end{array}$ & GGTGACAGGGAGGC \\
\hline E33 & $\begin{array}{c}\text { TTT } \\
\text { G }\end{array}$ & $\begin{array}{c}\text { ACATCATTA } \\
\text { AGGCTGGC } \\
\text { TCCCTG } \\
\end{array}$ & & & $\begin{array}{c}\text { GTGGGATAGCAGAT } \\
\text { ACTATAG }\end{array}$ \\
\hline 121 & TTTC & $\begin{array}{c}\text { ACGCTCTAG } \\
\text { CTCTGCAAC } \\
\text { GGAGC }\end{array}$ & & & \\
\hline 122 & TTTC & $\begin{array}{c}\text { ATAGAAAA } \\
\text { CCGTTCCAA } \\
\text { AATTCT }\end{array}$ & & & \\
\hline
\end{tabular}

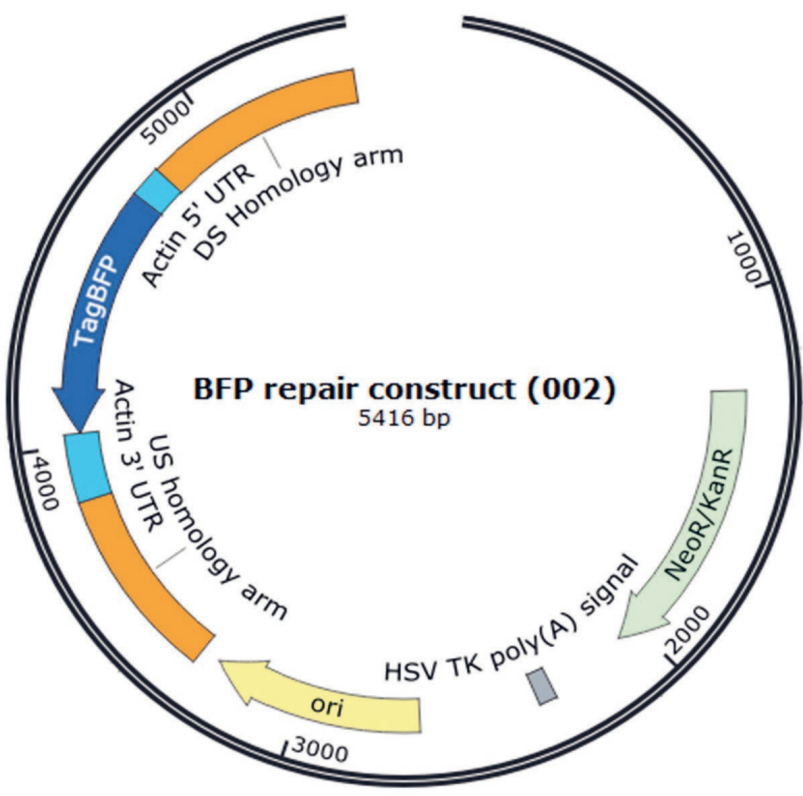

Figure S1. TagBFP repair construct with TagBFP in reverse orientation, flanked by S. domuncula actin locus 5' and 3' UTRs and US and DS $H A$, in plasmid backbone. 


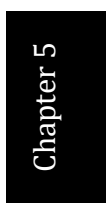


0 


\section{Chapter 6}

General Discussion:

Dawn of a New Era in Sponge Biotechnology

This chapter may be submitted for publication as:

Hesp, K., Martens, D.E., Wijffels, R.H., Pomponi, S.A. Dawn of a New

Era in Sponge Biotechnology (2021) 


\begin{abstract}
After more than 4 decades, the field of sponge biotechnology has finally overcome its most formidable obstacle: establishing a sponge cell line. Geodia barretti cells grow rapidly and continuously in optimized nutrient media, clearing the path for new lines of research. Whether to uncover how multicellular life evolved from unicellular organisms, study the origins of animal-microbe symbiosis, or develop production strains to industrialize new sponge-derived drugs and exploit the untapped biotechnological potential of the Porifera. Now is an exciting time in sponge biotechnology, so what's next?
\end{abstract}




\subsection{Introduction}

Many reports on sponge cell culture have stressed that sponges and spongederived microbes are a rich source of bioactive chemical compounds, and detailed how many of these compounds could be developed into novel drugs to combat cancer and bacterial, viral and fungal infections, to name a few ${ }^{35}$. All this potential was overshadowed by that seemingly insurmountable obstacle: obtaining sufficient supply for clinical trials. Difficulties with possible methods to obtain biomass were often discussed. Wild harvest of sponges is unsustainable, the complexity of many compounds impedes chemical synthesis, a poor understanding of the equally complex underlying metabolic pathways prevents recombinant production, and although aquaculture has been used with some success, this method does not have the benefit of a controlled environment to increase the often low concentrations in which compounds naturally occur. Producing these compounds in vitro with marine sponge cells has been proposed as an option to address these challenges, but the required cell line(s) did not yet exist, despite the efforts of many. Every new approach or technique helped us better understand how sponge cells behave in culture and some promising results were obtained. For example, PHA stimulated Hymeniacidon heliophila ${ }^{54}$ and Axinella corrugata ${ }^{47}$ cells to divide, and enriched archaeocytes of $H$. perleve proliferated both in primary cell ${ }^{101}$ and primmorph ${ }^{93}$ cultures. However, dividing cells could not be maintained or passaged consistently, either because cells became apoptotic ${ }^{57}$, cultures were overgrown by bacteria or fungi, or both ${ }^{60,94}$, 287. The field of sponge biotechnology was kept in abeyance, driven forward by the promise of untapped potential, held back by the inability to culture sponge cells.

\subsection{Similar tactics, different results?}

Adapting existing mammalian cell culture media such as M199, by adding salts to mimic sea water (Marine M199) and other ingredients such as growth factors to stimulate cells to divide, has been one of the main approaches taken to culture sponge cells ${ }^{81,84,90,95,97,98,116}$. In most studies using this approach the sponge cells did not divide. Recently, amino acid concentrations in Marine M199 were 
optimized for Dysidea etheria based on metabolic activity, resulting in a new medium, $\mathrm{M}^{103}$. Not long after, we discovered that cells of 9 other sponge species could divide rapidly in M1 medium. Finite cell lines were established for 3 species from the same genus, Geodia sp., G. neptuni, and G. barretti, which could be subcultured up to 5 times and could double maximally 7 times in M1 medium ${ }^{136}$ (Chapter 2 of this thesis). M1's successor OpM1 was also developed for $D$. etheria, but the added growth factors and other ingredients still did not stimulate the cells to divide (Munroe et al., in prep). When G. barretti cells were subcultured in OpM1, however, the first continuous marine sponge cell line was created, which continues to divide and has undergone nearly 100 doublings (Chapter 3) at the writing of this manuscript. Both studies represent major breakthroughs in sponge biotechnology. But why could cells from G. barretti and the other 8 species be cultured in M199-based media, while previous attempts to culture cells of other species in similar media did not succeed?

Unfortunate choices of model organisms (i.e., sponge species) seem to be the main reason sponge cells were not cultured successfully until now. Different sponge species, and even different individuals of the same species, can react vastly differently to their surroundings. This is illustrated by the different responses of cells from 12 sponge species when cultured in ASW, Marine M199 and M1 medium ${ }^{136}$ (Chapter 2 of this thesis). Cells of all 9 species that divided in M1 could also divide in Marine M199 and sometimes the cells reached the same density in both media. No previous attempts to culture cells from any of these 9 species in M199-based media were reported. Cells of 3 species did not respond to Marine M199 or M1 at all, of which 2 species, D. etheria and A. corrugata, had been the primary model organisms in sponge cell research in our research group. A. corrugata cells did not grow in M199 with adjusted salt concentrations, unless stimulated by $\mathrm{PHA}^{54}$. We hypothesize that OpM1 medium may induce $A$. corrugata cells to divide, since it also contains PHA. Both M1 and OpM1 were developed for $D$. etheria, but cells only became more metabolically active and did not divide ${ }^{103}$ (Munroe et al., in prep). It is possible that cells of the unresponsive species require nutrients that are not present in the media, or that concentrations of nutrients present are suboptimal, either too low or too high. 
Cells from 36 species (133 individuals) have been cultured in both Marine M199 and M1; of these, cells from 21 species (91 individuals) show an increase in cell number in $\mathrm{M} 1$ and/or Marine M199288. If unresponsive sponge species have common traits, this may reveal how to adapt the media to culture cells of these species.

\subsection{Sponge cells divide (incredibly) rapidly}

Our results demonstrate that sponge cells can divide extremely rapidly. $G$. barretti cells divide even faster in OpM1 than in $\mathrm{M} 1$, but in both media the doubling time is incredibly short: less than 1 hour in $M 1$, and less than 30 minutes in OpM1 (Chapter 3). Cells of some other species doubled even faster in M1 than G. barretti cells ${ }^{136}$ (Chapter 2 of this thesis). Doubling times in animal cells are notoriously long, for example, Chinese hamster ovary ( $\mathrm{CHO}$ ) cells divide approximately once per 20-24 hours. However, choanocytes from some sponge species have been reported to proliferate and turnover rapidly in situ ${ }^{289,290}$. Within 6 hours, $70.5 \%$ of all choanocytes in Mycale microsigmatosa had incorporated labelled bromodeoxyuridine (BrdU) while replicating their genome before dividing ${ }^{289}$. Sponge cells possess ${ }^{3}$ and express (Chapter 4 ) the same genes that regulate and drive cell cycling in all other animals. While cell cycling genes are highly conserved, it is possible that changes to the genes have increased the speed of sponges' replication machinery. However, even if that is the case, would it be physically possible for sponge cells to replicate their genomes more than once in 30 minutes? Sponges have compact genomes with an average haploid genome size of 196 million base pairs (Mbp), ranging between 40 and $616 \mathrm{Mbp}$ in different sponge species ${ }^{291}$. This is much smaller than the $3234 \mathrm{Mbp}$ haploid genome of a female human ${ }^{292}$, but the current fastest dividing eukaryotic organism, the yeast Kluyveromyces marxianus, has an even smaller genome (about $10 \mathrm{Mbp}$, but differs between strains) and the record-holding strain clocks in at 52 minutes per doubling ${ }^{293}$. Could sponge cells divide faster than $K$. marxianus despite having an at least 4 times larger genome? If so, how is this possible? 
It could be related to sponges' evolutionary position, as they provide a snapshot of life transitioning from uni- to multicellular organisms. Choanocytes, responsible for creating currents with their flagella and catching and taking up food particles, uncannily resemble unicellular choanoflagellates ${ }^{294,295}$. According to phylogenetic studies, choanoflagellates and animals likely had a last shared ancestor much like choanoflagellates alive today ${ }^{295-297}$. Some choanoflagellate species, such as Salpingoeca rosetta ${ }^{298}$ and Proterospongia choanojuncta ${ }^{299}$ can form simple colonies. It is not difficult to imagine how colonies of choanoflagellate-like unicellular ancestors could have transformed into chambers over time and made currents with their flagella to obtain food more efficiently. Abundant energy could allow some cells in the colony to specialize in other functions, and over time these colonies could give rise to multicellular organisms: sponges. Individuals of $S$. rosetta become diploid when nutrients are limited and revert to haploidy when transferred to nutrient-rich medium, presumably by dividing through meiosis ${ }^{300}$. Differentiated sponge cells are diploid, but choanocytes and archaeocytes can undergo meiosis to produce haploid gametes ${ }^{17}$. If changes in nutrient availability could trigger meiosis in sponge cells, this may occur when cells are placed in rich media such as M1 and OpM1 after being dissociated, cryopreserved and thawed in nutrient-deplete filtered-or artificial seawater.

Another option is alternate ploidy in G. barretti cells. Preliminary cell cycle analysis of $G$. barretti cells cultured in M1 suggested that the amount of DNA in the cell was quadrupled after the synthesis phase (Figure $1 A$ ), not doubled as one would expect (Figure 1B). This was not observed in dissociated cells of 5 other sponge species ${ }^{57}$, which could either be due to the medium, or part of the reason $G$. barretti and other Geodia species perform so well in culture. Other invertebrates, including the fresh-water polyp Hydra attenuata ${ }^{301}$ and upsidedown jellyfish Cassiopea sp. ${ }^{302}$, contain pools of cells with tetraploid (4n) G2 nuclei that divide when the animal is damaged. Such cell populations could contribute to or be responsible for sponges' potential to "heal" rapidly after mechanical damage ${ }^{303,304}$, although no significant pooling of tetraploid cells was 
A

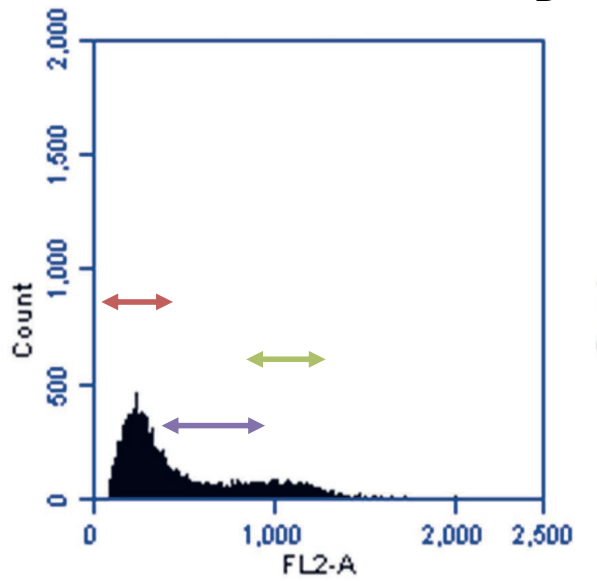

B

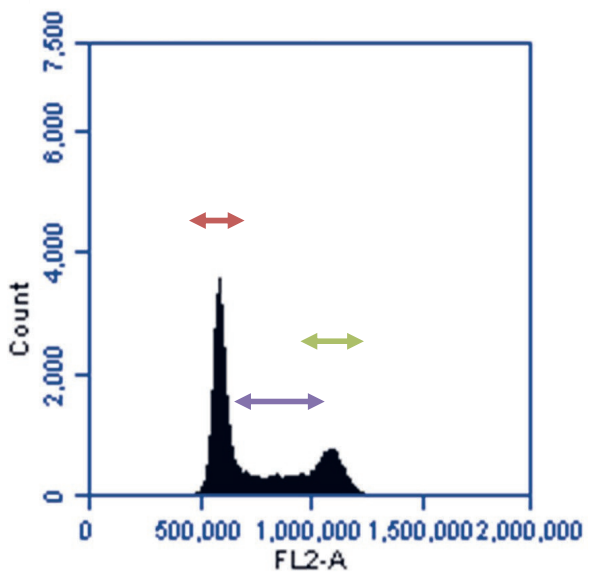

$\longleftrightarrow \mathrm{G} 0 / \mathrm{G} 1 \longleftrightarrow \mathrm{S} \longleftrightarrow \mathrm{G} 2 / \mathrm{M}$

Figure 1. Cell cycle analysis of $G$. barretti cells in $M 1$ for ( $t=15 \mathrm{~min})$ (A) and $\mathrm{CHO}$ cells in log phase (B). Red fluorescence of propidium iodide-stained DNA (FL2-A) is shown on the $x$-axis, while cells counts are on the y-axis. Cells in the G0/G1 peak (blue) have not started to replicate their genome, in the G2/M peak (purple) they are finished replicating the genome and in between are cells synthesizing DNA (S phase, green). G2/M phase cells usually contain twice the amount of DNA as GO/G1 phase cells and should fluoresce approximately twice as intensely, as is seen in the $\mathrm{CHO}$ cells (B). However, in G. barretti the G2/M phase cells fluoresce about $4 \mathrm{x}$ as intensely as the GO/G1 phase cells (A).

observed in 2 freshwater sponge species ${ }^{305}$. More elaborately investigating how the G. barretti cell cycle progresses in vitro and how many copies cells have of each chromosome before and after they divide in multiple passages, could uncover how sponge cells can proliferate so rapidly. Cultured G. barretti cells plateau quickly and then remain in the stationary phase for up to 7 days between passages, during which time the genome could be replicated so the cells can divide rapidly again when diluted in fresh medium. To test whether this is the case, cells could be subcultured before they reach their plateau. If the cells start dividing again straight away, that would suggest that they can replicate their genome very quickly. If not, the cells need more time to replicate their genomes before they can divide again, making it more likely that the cells divided without replicating the genome first. In OpM1, G. barretti cells reach a higher cell density 
than in M1. PHA is crucial for this difference (Chapter 3 ) and may induce mitosis in cells that were at the end of the G2/S phase and tetraploid when they were cryopreserved. Pifithrin- $\alpha$ and fetal bovine serum (FBS) in OpM1 also play important roles: our results show that both can help prevent apoptosis (Chapter 3) and FBS is also known to increase meiotic rates in bovine and hamster oocytes $^{306}$. Cells stop dividing quickly in both media ${ }^{136}$ (Chapter 2 of this thesis, Chapter 3 ), but start dividing again when diluted with fresh medium. This could be only because required nutrients are depleted, or because all cells have become haploid through meiosis or diploid through mitosis and start to synthesize DNA but do not divide due to depleted nutrients. It is unknown whether this occurs at the same speed as when they are first exposed to OpM1. Either way, depleted nutrients need to be identified by comparing nutrient concentrations in spent (used) and fresh medium. Increasing their concentrations may result in higher cell densities. Comparing amino acid concentrations in OpM1 before and after 7 days of culture showed that only tyrosine was depleted. However, tyrosine is the main precursor for melanin, which sponge cells in culture seem to produce not only while dividing, but also after they have plateaued ${ }^{136}$ (Chapter 2 of this thesis). Furthermore, doubling the amount of tyrosine in OpM1 medium did not lead to significantly higher cell densities (Hesp et al., unpublished data), suggesting at least 1 other nutrient is limiting. Studying the effect of various components, not only in the first passage but over time, will be critical for further optimizing the medium to culture cells of $G$. barretti or other species.

\subsection{Sponges \& immortality}

Currently, the continuous $G$. barretti cell line is continuing to grow and the cells have doubled nearly 100 times. It remains to be seen how many times sponge cells can maximally divide, but it is possible that no such limit exists in sponges. Our results (Chapter 4) confirmed previous reports that sponges express telomerase reverse transcriptase, or TERT ${ }^{59,89,91}$, the catalytic subunit of the ribonucleoprotein complex responsible for maintaining the length of life-span determining telomeres ${ }^{242}$. Overexpressing TERT is a well-known strategy to 
immortalize cells ${ }^{79,}{ }^{307}$. Despite expressing TERT in their cells, sponges have limited telomere length ${ }^{89}$, suggesting TERT is tightly regulated, possibly by other proteins that modulate its activity. Sponges do not seem to develop tumors ${ }^{246}$, which ironically is one of the main issues that has complicated developing a sponge cell line, as cancerous cells are easier to culture than normal healthy cells. As the longest living animals 247,248 , sponges can reach ages of hundreds, sometimes thousands of years. The title of oldest animal on Earth is held by a deep-water Monorhaphis chuni individual, dated to be about 11,000 years old ${ }^{247}$. Sponges can also remold and regenerate their tissues when damaged and form fully-functional sponges from fragments ${ }^{21}$ 23. For all the reasons mentioned, it has been hypothesized that sponges are naturally immorta| ${ }^{82,89,117,248}$. If so, they would need to be highly efficient at recognizing mutated DNA and either repairing the damage or eliminating the affected cells to prevent cells from accumulating deleterious mutations and become cancerous. While some have studied how sponges respond to and repair DNA damage ${ }^{250}$, little is known about the mechanisms and how they are different from and potentially improved compared to our own. These questions could be answered by exposing cultured sponge cells to mutagens and observing how they respond to and repair the resulting DNA damage compared to other animal cells. Furthermore, comparing and manually annotating (unknown) genes in the transcriptome of G. barretti cells in intact sponges, after being dissociated and cryopreserved, and while dividing in OpM1 medium (Chapter 4), may reveal novel or improved DNA repair pathways in sponges and how active these pathways are in cultured cells.

\subsection{Selection or adaptation?}

Studying how cultured sponge cells express genes differently than cells in intact sponges provides insights into the extensive changes that cells undergo in this process and how resilient sponge cells are (Chapter 4). In culture, cells phagocytose less than in intact sponges and shift more to taking up nutrients through diffusing and passive or active transporters across the plasma membrane (Chapter 4). It has been observed that on coral reefs, where dissolved organic matter (DOM) is plentiful, sponges obtain more carbon from DOM than 
by phagocytosing particulate organic matter $(\mathrm{POM})^{308}$. However, how fast the temperate water sponge Haliclona oculata grew in situ was found to be correlated with POM, but not DOM ${ }^{309}$. Moreover, different species were found to have widely different rates of taking up $\mathrm{POM}^{15}$, suggesting interspecies variation is once again an important factor. Whether cells of other sponge species also take up nutrients more through diffusion and less through phagocytosis remains to be seen. Cultured cells are less geared towards adhering to their surroundings, and more to life without an ECM, which correlates well with downregulated genes involved in forming an ECM and adhering to it at lower levels. Furthermore, while some genes involved in cell-cell adhesion were upregulated, G. barretti cells in culture downregulated their aggregation receptor, which is responsible for the impressive feat of sponge cells to form aggregates and eventually, fully functional new sponges after being fully dissociated ${ }^{233}, 234$. The roles of adhesion and aggregation in sponge cell cultures (Chapter 4) will need to be investigated further.

Cultured cells also metabolized nutrients differently, focusing more on making building blocks for new cells than breaking down lipids and glycogen for energy. (Chapter 4). But how are these changes happening? Are the culture conditions selecting a certain cell type? Or are cells adapting to match what their new environment requires? Different cell types present in sponge fragments seem to disappear in culture, as evidenced by downregulated genes that form cell typespecific structures such as flagellae. Furthermore, some changes were observed in genes that regulate differentiation and stemness (Chapter 4). If either of our hypotheses regarding the proliferative burst in the initial phase of the culture is correct, and if this is due to nutrients triggering meiosis or tetraploid cells responding to damage, it would make the most sense that the dividing cells are either dedifferentiated choanocytes or archaeocytes, as both cell types are pluripotent ${ }^{310}$ and can also undergo meiosis to produce gametes ${ }^{17}$. Cell typespecific protein markers have been discovered for various sponge cell types, including choanocytes ${ }^{311}$, archaeocytes ${ }^{312}$ and sclerocytes ${ }^{313}$, and expression analysis or immunohistochemistry or targeting these markers could determine which cell types are present before and after inoculating cultures, and after 
passaging. Although few changes were observed in the phyla from which active prokaryotic genes originated in sponge fragments, dissociated and cultured cells (Chapter4), a closer look at the changes in the microbial community as the culture progresses may reveal how the culture conditions affect obligate and facultative (endo)symbionts.

\subsection{First molecular tool in sponges: CRISPR-Cpf1}

While studying how sponge cells grow and express genes differently in culture, we also set out to develop molecular tools such as CRISPR-Cas gene editing in sponges. We successfully demonstrated that CRISPR-Cas can be used to edit the genome of $G$. barretti and insert sequences through homology-directed repair (HDR). The short single-stranded oligodeoxynucleotide (ssODN) donor seemed to mediate HDR more efficiently than the double-stranded (ds) plasmid donor template (Chapter 5), which is in line with previous reports ${ }^{314-316}$, but ssODNs are generally too short to insert coding sequences ${ }^{269}$. However, recently developed methods demonstrated efficient editing using longer ssODNs with short homology arms ${ }^{317,318}$. Since our short ssODN donor successfully inserted the intended sequence into the genome of $G$. barretti (Chapter 5), a long sSODN may be more efficient than a dsDNA donor. Another possibility is that the marker gene was inserted and not expressed, but the few cells that did express the gene fluoresced brightly (Chapter 5), making this less likely. If expression of the inserted gene did need to be improved, obtaining the promoter and terminator regions up- and downstream from a highly expressed G. barretti gene, such as actin, could be more effective than using the actin locus of $S$. domuncula ${ }^{270}$. Initially, we intended using CRISPR-Cas to immortalize cells by overexpressing genes stimulating cells to divide, or disrupting genes promoting apoptosis or cell cycle arrest. If sponge cells are indeed immortal, CRISPR-Cas will not be needed for this purpose. However, it can be used for various other purposes, for example, to screen for genes involved in metabolic pathways by disrupting them and observing the effect, to overexpress genes known to be involved in such pathways to create more productive strains, or to study how genes function and interact with each other in sponge cells from a never-before-seen perspective. 


\subsection{What's next?}

\subsubsection{Sponge cell life, death \& evolution}

Now that the first sponge cell lines are established and sponge cells can be continuously cultured, many new research opportunities have arisen. In vitro cultures can now be developed as model systems to study many fundamental aspects of sponge cells that are currently poorly understood. For example, whether sponge cells produce their bioactive compounds in vitro and how culture conditions influence productivity. How producing compounds relates to genes that are up- or downregulated can help unravel the complex pathways in which these compounds are produced and how these pathways can be manipulated to maximize product yields. This could also help determine whether compounds originate from the sponge, its microbial symbionts, or both ${ }^{94}$. Such model systems could also be used to predict how sponges may fare in an ocean affected by climate change by testing how cells respond to lower $\mathrm{pH}$ and higher temperatures. G. barretti cells tolerate culture temperatures as high as $22^{\circ} \mathrm{C}$, but other sponge species will likely react differently. Sponges have also emerged as models to study how animals live in symbiosis with microbes ${ }^{7}$ and observing the sponge holobiont in vitro can teach us more about how and why symbiotic relationships developed. Sponges can also illuminate how multicellular life evolved from unicellular organisms $s^{310,319,320}$ and thus about the origin of all animals, including humans.

\subsubsection{Expanding the molecular toolbox}

Once pathways producing potential biopharmaceuticals have been elucidated, molecular tools can be used to optimize sponge cell metabolism and develop production strains. Besides CRISPR-Cas gene editing, there are many other molecular techniques waiting to be developed, some of which have been previously used in sponges but were not (completely) successful ${ }^{58}$. For example, green fluorescent protein (GFP) was expressed in Ephydatia fluviatilis juveniles ${ }^{321}$ and $S$. domuncula explants ${ }^{270}$. Such techniques could overexpress genes without editing the genome, but the number of cells expressing the GFP gene was still limited and the expression only transient. Selectable markers on 
the plasmid with the gene of interest could solve both issues. Although no selection markers have been tested in sponge cells, various known selection agents can inhibit protein synthesis in a broad spectrum of eukaryotes. Agents that can kill sponge cells at low concentrations can be identified and a gene conferring resistance against the chosen agent can then be provided to select transformed cells and prevent cells from losing plasmids over time. Another promising technology is RNA interference (RNAi), in which double-stranded RNA molecules introduced into cells transiently silence expression of a target gene. Such temporary gene silencing allows the study of gene function without altering the gene itself. RNAi has been demonstrated in sponge tissues ${ }^{322}$, but has yet to be established in sponge cell culture.

In cases where compounds are produced by sponge species that cannot be cultured, or through pathways that cannot be elucidated or difficult to manipulate, hybridoma technology could be used to develop production strains. This technique is traditionally used to produce monoclonal antibodies, by fusing antibody-producing B cells with rapidly dividing tumor cells to create hybrid cells (hybridomas) that both produce the desired antibody and divide rapidly ${ }^{323}$. Similarly, fusing rapidly dividing cells with sponge cells that produce pharmaceutically relevant compounds could result in a hybrid cell line with both desirable features. It has been shown that cells of different marine sponge species can be fused successfully ${ }^{55}$. Sponge cell lines could be ideal fusion partners to create sponge hybridomas (Figure 2).

A

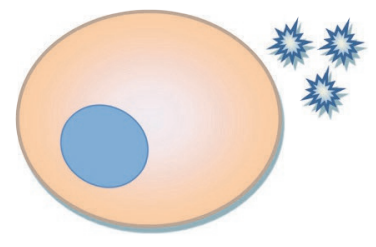

B

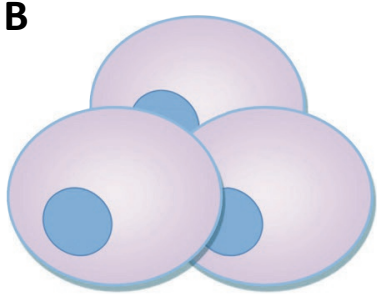

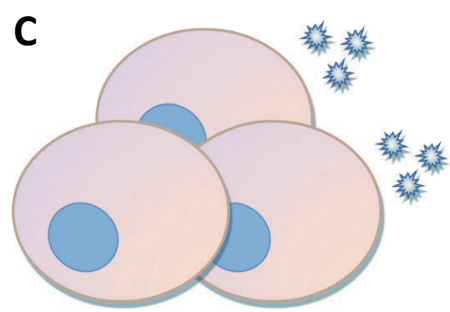

Figure 2. Sponge hybridoma principle. Uncultivable sponge cells that produce compounds of interest (A) can be fused with a sponge cell line from another species (B) to create a hybrid cell line (C) that can both divide rapidly and produce the compound. 


\subsubsection{Culturing sponge cells on industrial scale}

Methods developed to create the $G$. barretti cell line may function as a road map to establish cell lines for other sponge species as well. However, experience teaches us that each sponge species and individual is different ${ }^{103,136}$. Developing new cell lines will entail carefully assessing and screening source material and optimizing media and culture conditions for each species. Once established, sponge cell lines may be developed into production strains with the newly obtained molecular tools and knowledge about sponge cells and their metabolic pathways. These production strains could provide sufficient supply for clinical trials and subsequently produce newly developed drugs for the pharmaceutical market. However, before sponge cell lines can produce compounds on an industrial scale, a production process needs to be designed and developed. Culture parameters like vessel type, temperature, oxygen and carbon dioxide levels, $\mathrm{pH}$, and agitation should be optimized specifically for each species and envisioned products to increase maximum cell density and yield. Most biopharmaceuticals, such as monoclonal antibodies in $\mathrm{CHO}$ cells, are produced in large (up to 25,000 liter) stirred-tank bioreactors (Figure 3A) ${ }^{324}$. Preliminary experiments in our group have indicated that sponge cells do not grow well with rotary agitation (Hesp et al., unpublished data) and although they can be released by gentle pipetting, cells do adhere somewhat to the culture vessel. Since suspension cell cultures are easily scalable, especially when aerated to improve gas transfer, it would be useful if sponge cell lines could proliferate while suspended in medium. Shear protectants such as Pluronic F-68 ${ }^{325}$ or dextran ${ }^{326}$ can prevent damage to cells caused by agitating and aerating cells. Until sponge cell suspension culture has been developed, reactors employing different strategies to increase surface area for cells to adhere could be better suited for culturing sponge cells. Examples are microcarrier (Figure 3B) ) $^{32-331}$ and hollow-fiber (Figure $3 \mathrm{C}$ ) reactors ${ }^{332}$. Testing various reactor types on a small scale can help identify the best design for culturing sponge cells at industrial scale. 
A

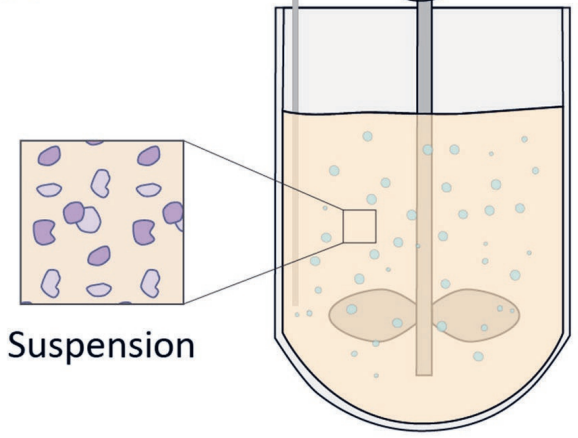

B

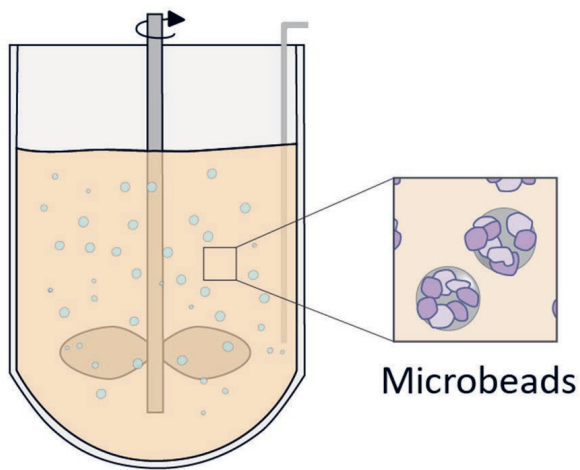

C

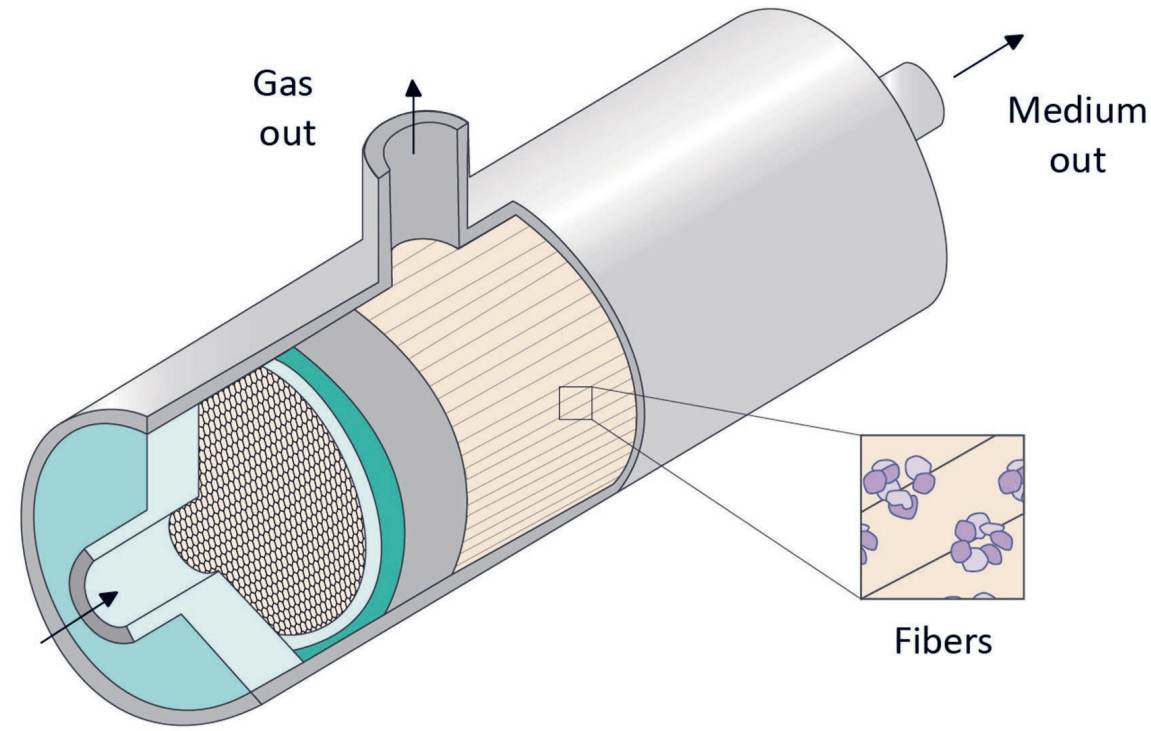

gas in

Figure 3. Different types of bioreactors that could be used to culture sponge cells at a larger scale. Options include classic stirred-tank bioreactors with cells either suspended in medium (A) or adhered to microcarrier beads (B), or novel reactor types such as hollow-fiber reactors (C) where medium passes through permeable fibers that cells can adhere to on the in-or outside.

\subsubsection{Axenic sponge cell culture?}

An interesting question is whether sponge cell lines and production processes could, and should, be axenic. As holobionts, sponges associate closely with their microbial symbionts ${ }^{7}$. Some species may have obligate (endo)symbionts, and both the host and its symbionts are likely required to produce certain compounds ${ }^{333}$. Mixed cultures may be the only option to produce these 
compounds, which would likely require the cells to be cultured with different or no antimicrobials. Work ongoing in our group (Conkling \& Pomponi, unpublished) shows cells of $G$. neptuni can be cultured without antimicrobials. However, a balance will need to be struck between accommodating symbionts and keeping contaminating microbes at bay. Furthermore, extra care needs to be taken in downstream processing to ensure no microbes are present in the final product. Compounds that are cytostatic, antibacterial or otherwise biologically active could become toxic to sponge cells or their obligate symbionts at high concentrations, which may limit maximum product yields on biomass or require removing product while cells are growing. These factors must all be considered to successfully produce biopharmaceuticals with sponge cell lines.

\subsection{Concluding remarks}

Over the last few years, we have made important progress and learned much about sponge cells and how to culture them. We have shown that sponge cells of multiple species can divide extremely rapidly in M1 medium and have established finite cell lines for 3 Geodia species. Culturing G. barretti in M1's successor, OpM1 medium, resulted in the first continuous marine sponge cell line, which may already be immortal. Examining genes differentially expressed by sponge cells in vivo and in vitro has given us unprecedented insight into their inner workings, and we have taken the first step towards developing a molecular toolbox for these organisms. Of course, many new challenges await us in the uncharted territories ahead, like unraveling how multicellular life evolved, elucidating and manipulating complex metabolic pathways, and developing and optimizing production strains and processes for sponge-derived biopharmaceuticals. The means and opportunity to tackle these challenges are now available. It is the dawn of a new era in sponge biotechnology. 


\section{Summary}

Sponges are ancient filter-feeding animals that seem to have barely changed since they evolved over 600 million years ago. Water enters through pores connecting to a myriad of channels, pumped through chambers where choanocytes take up particles or dissolved nutrients, and then expelled through oscula. However, this seemingly simple system conceals a complex conglomerate of chemical compounds, many of which are biologically active. These chemicals allow sponges to interact with their surroundings and protect themselves. For example, antibacterial and antiviral compounds ward off potential pathogens, while cytostatic compounds prevent other competing organisms from growing. When such compounds were first discovered, sponges were quickly recognized as a treasure trove of potential new drugs. However, a bottleneck emerged that would stymie sponge cell culture for decades: obtaining enough biomass to produce compounds at a large enough scale for clinical trials. Many studies focused on developing sponge cell lines, which would provide a scalable platform to produce compounds in a controlled environment. Despite all efforts and promising results, no sponge cell lines were established.

In Chapter 2 we reported a long-awaited breakthrough: cells of 9 sponge species divided rapidly in amino acid-optimized nutrient medium $M 1$, based on mammalian cell culture medium 199 (M199). The fastest dividing cells doubled in under an hour. Cells of species that responded to M1 medium could also divide in the more basal marine-adjusted M199, albeit usually slower and to a lower density. Among these species were 3 members of the genus Geodia, G. neptuni, Geodia sp., and G. barretti, which showed most consistent results between different individuals. The Geodia spp. were subcultured 3 - 5 times over a period of 21 - 35 days and reached an average total of 6 population doublings. All 3 species were able to proliferate at both $4{ }^{\circ} \mathrm{C}$, the temperature used for $G$. barretti, and $22^{\circ} \mathrm{C}$, at which $\mathrm{G}$. neptuni and Geodia sp. were incubated. The finite cell lines we developed represented the first real leads to develop stable or continuous marine sponge cell lines. 
We followed up on this lead for $G$. barretti in Chapter 3 and established the first continuous marine sponge cell line. Cells of 3 individuals $G$. barretti were cultured in OpM1 medium, the successor of M1 with added growth factors, lipids, vitamins, and other nutrients. In OpM1, G. barretti cells proliferated even more rapidly and to a higher density than in M1 medium. We analyzed the impact of the individual components in OpM1 and determined that phytohemagglutinin (PHA) was a critical component. Besides this finding, all-butone of the added nutrient mixtures in OpM1 contributed to the differences in cell division between OpM1 and M1. In subculturing experiments, G. barretti cells of 3 individuals could double nearly 100 times, compared to 5 doublings cells of the same individuals reached in M1. The maximum number of doublings of the $G$. barretti cell line has yet to be determined. Subcultured cells could be cryopreserved and thawed to inoculate new cultures. These results brought us one step closer to sponge cells producing biopharmaceuticals at industrial scale. Although we now had proliferating sponge cells, we still did not know much about what stimulates them to divide, how they respond to nutrients, or deal with stress, among others. In Chapter 4 we started to bridge this knowledge gap by comparing genes expressed by $G$. barretti cells in fragments of an intact sponge, in cells after being dissociated and cryopreserved, and in cells cultured in OpM1 medium. Cells in all 3 states expressed genes that drive the cell cycle and prevent apoptosis at equal levels. Telomerase reverse transcriptase, the gene that determines the number of times the genome can be replicated, was also expressed in all samples, providing further evidence that sponge cells may be naturally immortal. Few differences were observed between sponge fragments and dissociated cells, suggesting dissociating cells does not strongly affect them. Genes expressed in cultured cells and sponge fragments were most different, showing the extensive changes needed for sponge cells to adjust to in vitro culture, either because cells actively adapt or because a specific cell type is selected by culture conditions. Cultured cells reorganized their cytoskeleton, downregulated genes needed to adhere to the extracellular matrix, synthesized more proteins and lipids, and broke down fewer lipids and glycogen reserves for energy, likely favoring soluble energy sources in the medium. Phagocytosis was 
also downregulated, further indicating that cells use soluble energy sources that can diffuse or be transported across the plasma membrane, either because cells adapt or because the culture conditions favor cell types with these traits. These results gave us unprecedented insight into the inner workings of sponge cells.

In Chapter 5 we demonstrated that cultured G. barretti cells can be genetically modified with the CRISPR/Cas12a system. We used CRISPR/Cas12a to introduce a double-stranded break at a target site in the G. barretti 2',5'-oligoadenylate synthetase gene, and inserted a scrambled DNA sequence through homologydirected repair by providing a single-stranded DNA donor with short homology arms. A longer double-stranded DNA donor was used to insert and express blue fluorescent marker gene TagBFP, although efficiencies were low. Introducing longer constructs as single-stranded donors may allow more efficient gene editing. Our results represent an important step towards developing a molecular tool box for sponge cells.

Chapter 6 reflects on what we have learned in the previous chapters, which questions have arisen from this new knowledge, and what next steps should be taken. Our results seem to implicate bad luck in choosing model organisms as the main reason why sponge cells were not cultured successfully before. Sponge cells can divide incredibly rapidly, and we hypothesize that the cells may be dividing without replicating their genome, either because tetraploid cells are present to immediately start regenerating the sponge after damage, or because the sudden change in the presence of nutrients may trigger the cells to undergo meiosis, a trait found in a phylogenetically-related group, the unicellular choanoflagellates. Active telomerase found in sponge cells and the age sponges are reported to reach in the wild suggest sponges may be naturally immortal. Studying telomere length in the $G$. barretti cell line may help shed light on this matter, as well as elucidating how sponges repair DNA damage to maintain genomic integrity during their long lifespan. If sponge cells are indeed immortal, we will not need CRISPR-Cas12a to immortalize sponge cells. However, this versatile tool has many other uses in developing production strains from sponge cells, for example by upregulating metabolic pathways to increase product yields or knocking out other pathways to direct resources to the product. 
Finally, we discuss the future of sponge biotechnology. Now that we can culture sponge cells consistently and possibly continuously, many new lines of research have opened up. Methods used to develop the G. barretti cell line can be applied to other species. However, high levels of inter- and intraspecies variation in sponges means model organisms and source material will need to be assessed carefully using preliminary experiments, and establishing cell lines will require species-specific optimizing of media and culture conditions. Sponge cell lines are an ideal model system for all kinds of experiments, for example to predict the impact of warmer and more acidic oceans due to climate change, study animalmicrobe (endo)symbiosis, or determine how bioactive compounds are produced. Understanding these pathways will help improve yields and produce sufficient supply of bioactive compounds for clinical trials and treatment. Before sponge cell lines can produce bioactive compounds on an industrial scale, different bioreactors will need to be tested at a small scale to determine the most efficient design. If sponge cell lines can be axenic and how this would affect desired compounds still needs to be determined. Many new challenges await, but we now have the required tools and knowledge to overcome these challenges. It is the dawn of a new era in sponge biotechnology. 


\section{References}

1. Worheide, G., Sole-Cava, A. M. \& Hooper, J. N. Biodiversity, molecular ecology and phylogeography of marine sponges: patterns, implications and outlooks. Integr. Comp. Biol. 45, 377385 (2005).

2. Hogg, M. M. et al. in Deep-Sea Sponge Grounds: Reservoirs of Biodiversity (UNEP-WCMC, Cambridge, 2010).

3. Srivastava, M. et al. The Amphimedon queenslandica genome and the evolution of animal complexity. Nature 466, 720-726 (2010).

4. Morris, P. J. The Developmental Role of the Extracellular Matrix Suggests a Monophyletic Origin of the Kingdom Animalia. Evolution 47, 152-165 (1993).

5. Sperling, E. A., Peterson, K. J. \& Pisani, D. Phylogenetic-signal dissection of nuclear housekeeping genes supports the paraphyly of sponges and the monophyly of Eumetazoa. Mol. Biol. Evol. 26, 22612274 (2009).

6. Hentschel, U., Usher, K. M. \& Taylor, M. W. Marine sponges as microbial fermenters. FEMS Microbiol. Ecol. 55, 167-177 (2006).

7. Pita, L., Fraune, S. \& Hentschel, U. Emerging Sponge Models of Animal-Microbe Symbioses. Front. Microbiol. 7, 2102 (2016).

8. Brinkmann, C. M., Marker, A. \& Kurtböke, D. I. An Overview on Marine Sponge-Symbiotic Bacteria as Unexhausted Sources for Natural Product Discovery. Diversity 9 (2017).

9. Pomponi, S. A. The bioprocess-technological potential of the sea. Journal of Biotechnology 70, 5-13 (1999).

10. Leal, M. C., Puga, J., Serôdio, J., Gomes, N. C. M. \& Calado, R. Trends in the Discovery of New Marine Natural Products from Invertebrates over the Last Two Decades: Where and What Are We Bioprospecting? PLOS ONE 7, e30580 (2012).

11. Blunt, J. W., Copp, B. R., Keyzers, R. A., Munro, M. H. G. \& Prinsep, M. R. Marine natural products. Natural Product Reports 31, 160-258 (2014).

12. Mehbub, M. F., Lei, J., Franco, C. \& Zhang, W. Marine sponge derived natural products between 2001 and 2010: trends and opportunities for discovery of bioactives. Mar. Drugs 12, 4539-4577 (2014).

13. Anjum, K. et al. Marine Sponges as a Drug Treasure. Biomolecules \& therapeutics 24, 347-362 (2016).

14. Lavrov, A. I. \& Kosevich, I. A. Sponge cell reaggregation: mechanisms and dynamics of the process. Ontogenez 45, 250-271 (2014). 
15. Koopmans, M., Martens, D. \& Wijffels, R. H. Growth efficiency and carbon balance for the sponge Haliclona oculata. Mar. Biotechnol. (NY) 12, 340-349 (2010).

16. Gili, J. M. \& Coma, R. Benthic suspension feeders: their paramount role in littoral marine food webs. Trends Ecol. Evol. 13, 316-321 (1998).

17. Funayama, N. The stem cell system in demosponges: insights into the origin of somatic stem cells. Dev. Growth Differ. 52, 1-14 (2010).

18. Ruppert, E. E., Fox, R. S. \& Barnes, R. D. in Invertebrate Zoology: A Functional Evolutionary Approach

(Brooks/Cole-Thomson Learning, Belmont, Calif, 2004).

19. Leys, S. P., Nichols, S. A. \& Adams, E. D. Epithelia and integration in sponges. Integr. Comp. Biol. 49, 167-177 (2009)

20. Maldonado, M. \& Riesgo, A. Reproduction in the phylum Porifera: a synoptic overview. Treballs de la SCB 59, 29-49 (2008).

21. Ereskovsky, A., Geronimo, A. \& Perez, T. Asexual and puzzling sexual reproduction of the Mediterranean sponge Haliclona fulva (Demospongiae): life cycle and cytological structures. Invertebrate Biology, Wiley 136, 403-421 (2017).

22. Fell, P. E. in Reproductive Biology of Invertebrates (eds Adiyodi, K. G. \& Adiyodi, R. G.) 1-44 (Oxford \& IBH Publishing Co., New Delhi, 1993).

23. Ereskovsky, A. in The comparative embryology of sponges (Springer Verlag, Dordrecht, Heidelberg, London, New York, 2010).

24. Proksch, P. Defensive roles for secondary metabolites from marine sponges and sponge-feeding nudibranchs. Toxicon 32, 639-655 (1994).

25. Amade, P., Pesando, D. \& Chevolot, L. Antimicrobial activities of marine sponges from French Polynesia and Brittany. Marine Biology 70, 223-228 (1982).

26. Amade, P., Charroin, C., Baby, C. \& Vacelet, J. Antimicrobial activities of marine sponges from the Mediterranean Sea

. Marine Biology 94, 271-275 (1987).

27. Bergquist, P. R. \& Bedford, J. J. The incidence of antibacterial activity in marine Demospongiae; systematic and geographic considerations. Marine Biology 46, 215-221 (1978).

28. Rohde, S., Nietzer, S. \& Schupp, P. J. Prevalence and Mechanisms of Dynamic Chemical Defenses in Tropical Sponges. PLoS One 10, e0132236 (2015).

29. Wahl, M. Fouling and antifouling: some basic aspects. I. Marine epibiosis. Marine Ecology Progress Series 58, 175-189 (1989).

30. Kelly, S. R., Garo, E., Jensen, P. R., Fenical, W. \& Pawlik, J. R. Effects of Caribbean sponge secondary metabolites on bacterial surface colonization. Aquat. Microb. Ecol. 40: 191-203. Aquatic Microbial Ecology 40, 191-203 (2005). 
31. Tsoukatou, M., Hellio, C., Vagias, C., Harvala, C. \& Roussis, V. Chemical defense and antifouling activity of three Mediterranean sponges of the genus Ircinia. Z. Naturforsch. C. 57, 161-171 (2002).

32. Singh, A. \& Thakur, N. L. Significance of investigating allelopathic interactions of marine organisms in the discovery and development of cytotoxic compounds. Chem. Biol. Interact. 243, 135147 (2016).

33. Burns, E., Ifrach, I., Carmeli, S., Pawlik, J. R. \& Ilan, M. Comparison of anti-predatory defenses of Red Sea and Caribbean sponges. I. Chemical defense. Mar Ecol Prog Ser. 252: 105-114. Marine Ecology Progress Series 252, 105-114 (2003).

34. Pawlik, J. R., Charnas, B., Toonen, R. J. \& Fenical, W. Defenses of Caribbean sponges against predatory reef fish. I. Chemical deterrency. Marine Ecology Progress Series 127, 183-194 (1995).

35. Rangel, M. \& Falkenberg, M. in An overview of the marine natural products in clinical trials and on the market 421-428, 2015).

36. Abdelmohsen, U. R. et al. Potential of marine natural products against drug-resistant fungal, viral, and parasitic infections. Lancet Infect. Dis. 17, e30-e41 (2017).

37. Shinde, P., Banerjee, P. \& Mandhare, A. Marine natural products as source of new drugs: a patent review (2015-2018). Expert Opinion on Therapeutic Patents 29, 283-309 (2019).

38. Pomponi, S. A. The oceans and human health: the discovery and development of marine-derived drugs. Oceanography 14, 78-87 (2001).

39. Calcabrini, C., Catanzaro, E., Bishayee, A., Turrini, E. \& Fimognari, C. Marine Sponge Natural Products with Anticancer Potential: An Updated Review. Mar. Drugs 15, 10.3390/md15100310 (2017).

40. Ruiz-Torres, V. et al. An Updated Review on Marine Anticancer Compounds: The Use of Virtual Screening for the Discovery of Small-Molecule Cancer Drugs. Molecules 22,

10.3390/molecules22071037 (2017).

41. Liu, M. et al. Potential of marine natural products against drug-resistant bacterial infections. Lancet Infect. Dis. (2019).

42. Sagar, S., Kaur, M. \& Minneman, K. P. Antiviral lead compounds from marine sponges. Mar. Drugs 8, 2619-2638 (2010).

43. El-Hossary, E. M. et al. Antifungal potential of marine natural products. Eur. J. Med. Chem. 126, 631-651 (2017).

44. Muller, W. E. et al. Avarol, a cytostatically active compound from the marine sponge Dysidea avara. Comp. Biochem. Physiol. C. 80, 47-52 (1985).

45. Muller, W. E. et al. Avarol-induced DNA strand breakage in vitro and in Friend erythroleukemia cells. Cancer Res. 47, 6565-6571 (1987). 
46. Balasubramanian, S. et al. A New Bioactive Compound From the Marine Sponge-Derived Streptomyces sp. SBT348 Inhibits Staphylococcal Growth and Biofilm Formation. Front. Microbiol. 9, 1473 (2018).

47. Pomponi, S. A. \& Willoughby, R. in Aquatic invertebrate cell culture (eds Mothersill, C. \& Austin, B.) 323-336 (Springer-Verlag, Berlin, 2000).

48. BioScience. 46 (1999).

49. Jordan, M. A. et al. The primary antimitotic mechanism of action of the synthetic halichondrin E7389 is suppression of microtubule growth. Mol. Cancer. Ther. 4, 1086-1095 (2005).

50. Bai, R. L. et al. Halichondrin B and homohalichondrin B, marine natural products binding in the vinca domain of tubulin. Discovery of tubulin-based mechanism of action by analysis of differential cytotoxicity data. J. Biol. Chem. 266, 15882-15889 (1991).

51. Duckworth, A. R. \& Battershill, C. Sponge aquaculture for the production of biologically active metabolites: the influence of farming protocols and environment. Aquaculture 221, 311-329 (2003).

52. Page, M. J., Northcote, P. T., Webb, V. L., Mackey, S. \& Handley, S. J. Aquaculture trials for the production of biologically active metabolites in the New Zealand sponge Mycale hentscheli (Demospongiae: Poecilosclerida). Aquaculture 250, 256-269 (2005).

53. Pomponi, S. A., Willoughby, R. \& Kelly-Borges, M. in Molecular Approaches to the Study of the Ocean 423-433 (Springer Netherlands, 1998).

54. Pomponi, S. A., Willoughby, R., Kaighn, M. E. \& Wright, A. E. Development of techniques for in vitro production of bioactive natural products from marine sponges (Proceedings from the World Congress on in vitro biology, 1997b).

55. Pomponi, S. A., Jevitt, A., Patel, J. \& Diaz, M. C. Sponge hybridomas: applications and implications. Integr. Comp. Biol. 53, 524-530 (2013).

56. Pomponi, S. A. Biology of the Porifera: Cell culture. Canadian Journal of Zoology 84, 167-174 (2006).

57. Schippers, K. J., Martens, D. E., Pomponi, S. A. \& Wijffels, R. H. Cell cycle analysis of primary sponge cell cultures. In Vitro Cell. Dev. Biol. Anim. 47, 302-311 (2011).

58. Grasela, J. J., Pomponi, S. A., Rinkevich, B. \& Grima, J. Efforts to develop a cultured sponge cell line: revisiting an intractable problem. In Vitro Cell. Dev. Biol. Anim. 48, 12-20 (2012).

59. Pomponi, S. A., Thompson, J., Bottesch, J. \& Sennett, S. Methods for extending the life span of marine invertebrate cells. 11/796,862 (2007).

60. Rinkevich, B. Marine invertebrate cell cultures: new millennium trends. Mar. Biotechnol. (NY) 7, 429-439 (2005).

61. Lopez-Lazaro, M. Two preclinical tests to evaluate anticancer activity and to help validate drug candidates for clinical trials. Oncoscience 2, 91-98 (2015). 
62. Deshmukh, R. S., Kovacs, K. A. \& Dinnyes, A. Drug discovery models and toxicity testing using embryonic and induced pluripotent stem-cell-derived cardiac and neuronal cells. Stem Cells Int. 2012, 379569 (2012).

63. Allen, D. D. et al. Cell lines as in vitro models for drug screening and toxicity studies. Drug Dev. Ind. Pharm. 31, 757-768 (2005).

64. Dumont, J., Euwart, D., Mei, B., Estes, S. \& Kshirsagar, R. Human cell lines for biopharmaceutical manufacturing: history, status, and future perspectives. Crit. Rev. Biotechnol. 36, 1110-1122 (2016).

65. Nomoto, H. et al. Bio-artificial bone formation model with a radial-flow bioreactor for implant therapy-comparison between two cell culture carriers: porous hydroxyapatite and beta-tricalcium phosphate beads. Hum. Cell 32, 1-11 (2019).

66. Cal, E., Cetintas, V. B., Boyacioglu, H. \& Guneri, P. Cytotoxicity of Dental Implants: The Effects of Ultrastructural Elements. Int. J. Oral Maxillofac. Implants 32, 1281-1287 (2017).

67. Frohlich, M. et al. Tissue engineered bone grafts: biological requirements, tissue culture and clinical relevance. Curr. Stem Cell. Res. Ther. 3, 254-264 (2008).

68. Shukla, S. J., Huang, R., Austin, C. P. \& Xia, M. The future of toxicity testing: a focus on in vitro methods using a quantitative high-throughput screening platform. Drug Discov. Today 15, 997-1007 (2010).

69. Uysal, O., Sevimli, T., Sevimli, M., Gunes, S. \& Eker Sariboyaci, A. Chapter 17 - Cell and Tissue Culture: The Base of Biotechnology. , 391-429 (2018).

70. Lucey, B. P., Nelson-Rees, W. A. \& Hutchins, G. M. Henrietta Lacks, HeLa cells, and cell culture contamination. Arch. Pathol. Lab. Med. 133, 1463-1467 (2009).

71. De Coppi, P. et al. Isolation of amniotic stem cell lines with potential for therapy. Nat. Biotechnol. 25, 100-106 (2007).

72. Wagers, A. J. \& Weissman, I. L. Plasticity of adult stem cells. Cell 116, 639-648 (2004).

73. van der Valk, J. et al. Optimization of chemically defined cell culture media--replacing fetal bovine serum in mammalian in vitro methods. Toxicol. In. Vitro. 24, 1053-1063 (2010).

74. Price, P. J. Best practices for media selection for mammalian cells. In Vitro Cell. Dev. Biol. Anim. 53, 673-681 (2017).

75. Li, L. et al. Heparin promotes suspension adaptation process of CHO-TS28 cells by eliminating cell aggregation. Mol. Biotechnol. 47, 9-17 (2011).

76. $\mathrm{Xu}, \mathrm{X}$. et al. The genomic sequence of the Chinese hamster ovary (CHO)-K1 cell line. Nat. Biotechnol. 29, 735-741 (2011).

77. Park, J. Y. et al. Development of suspension cell culture model to mimic circulating tumor cells. Oncotarget 9, 622-640 (2017). 
78. Uysal, A. \& Kaaden, O. R. Establishment of cell lines from bovine brain. Arch. Virol. Suppl. 7, 303308 (1993).

79. Garbe, J. C. et al. Immortalization of normal human mammary epithelial cells in two steps by direct targeting of senescence barriers does not require gross genomic alterations. Cell. Cycle $\mathbf{1 3}$ 3423-3435 (2014).

80. Carter, M. \& Shieh, J. C. Chapter 13 - Cell Culture Techniques. , 281-296 (2010).

81. Pomponi, S. A. \& Willoughby, R. in Sponges in time and space 395-400 (Balkema, Rotterdam, 1994).

82. Koziol, C., Borojevic, R., Steffen, R. \& Muller, W. E. Sponges (Porifera) model systems to study the shift from immortal to senescent somatic cells: the telomerase activity in somatic cells. Mech. Ageing Dev. 100, 107-120 (1998).

83. Muller, W. E. et al. Establishment of a primary cell culture from a sponge: primmorphs from Suberites domuncula. Marine Ecology Progress Series 178, 205-219 (1999).

84. Krasko, A. et al. Expression of silicatein and collagen genes in the marine sponge Suberites domuncula is controlled by silicate and myotrophin. Eur. J. Biochem. 267, 4878-4887 (2000).

85. Valisano, L., Bavestrello, G., Giovine, M., Arillo, A. \& Cerrano, C. Effect of iron and dissolved silica on primmorphs of Petrosia ficiformis (Poiret, 1789). Chem. Ecol. 23, 233-241 (2007).

86. Mussino, F. et al. Primmorphs cryopreservation: a new method for long-time storage of sponge cells. Mar. Biotechnol. (NY) 15, 357-367 (2013).

87. Sipkema, D. et al. Primmorphs from seven marine sponges: formation and structure. J. Biotechnol. 100, 127-139 (2003).

88. Sipkema, D., Tramper, J., Osinga, R. \& Wijffels, R. H. in Marine biotechnology: an overview of leading fields 56-63 (Ifremer, Plouzane, France, 2002).

89. Custodio, M. R. et al. Primmorphs generated from dissociated cells of the sponge Suberites domuncula: a model system for studies of cell proliferation and cell death. Mech. Ageing Dev. 105, 45-59 (1998).

90. Zhang, X. Y., Zhao, Q. Y., Xue, S. \& Zhang, W. Bioactive compounds from marine sponges and cell culture of marine sponges. Sheng Wu Gong Cheng Xue Bao 18, 10-15 (2002).

91. Muller, W. E. et al. Application of cell culture for the production of bioactive compounds from sponges: synthesis of avarol by primmorphs from Dysidea avara. J. Nat. Prod. 63, 1077-1081 (2000).

92. Krasko, A. et al. Iron induces proliferation and morphogenesis in primmorphs from the marine sponge Suberites domuncula. DNA Cell Biol. 21, 67-80 (2002).

93. Zhang, X., Cao, X., Zhang, W., Yu, X. \& Jin, M. Primmorphs from archaeocytes-dominant cell population of the sponge hymeniacidon perleve: improved cell proliferation and spiculogenesis. Biotechnol. Bioeng. 84, 583-590 (2003). 
94. Sipkema, D. et al. Large-scale production of pharmaceuticals by marine sponges: sea, cell, or synthesis? Biotechnol. Bioeng. 90, 201-222 (2005).

95. Zhang, W. et al. Optimizing the formation of in vitro sponge primmorphs from the Chinese sponge Stylotella agminata (Ridley). J. Biotechnol. 100, 161-168 (2003).

96. Richelle-Maurer, E. et al. Primary cultures from the marine sponge Xestospongia muta (Petrosiidae, Haplosclerida). Journal of Biotechnology 100, 169-176 (2003).

97. Zhang, X., Le Pennec, G., Steffen, R., Muller, W. E. \& Zhang, W. Application of a MTT assay for screening nutritional factors in growth media of primary sponge cell culture. Biotechnol. Prog. 20, 151-155 (2004).

98. Zhao, Q., Zhang, W., Jin, M., Yu, X. \& Deng, M. Formulation of a basal medium for primary cell culture of the marine sponge Hymeniacidon perleve. Biotechnol. Prog. 21, 1008-1012 (2005).

99. Krasko, A. et al. Ethylene modulates gene expression in cells of the marine sponge Suberites domuncula and reduces the degree of apoptosis. J. Biol. Chem. 274, 31524-31530 (1999).

100. Le Pennec, G. et al. Cultivation of primmorphs from the marine sponge Suberites domuncula: morphogenetic potential of silicon and iron. J. Biotechnol. 100, 93-108 (2003).

101. Sun, L., Song, Y., Qu, Y., Yu, X. \& Zhang, W. Purification and in vitro cultivation of archaeocytes (stem cells) of the marine sponge Hymeniacidon perleve (Demospongiae). Cell Tissue Res. 328, 223237 (2007).

102. Munroe, S., Martens, D. E., Sipkema, D. \& Pomponi, S. A. Comparison of Cryopreservation Techniques for Cells of the Marine Sponge Dysidea etheria. Cryo Letters 39, 269-278 (2018).

103. Munroe, S., Sandoval, K., Martens, D. E., Sipkema, D. \& Pomponi, S. A. Genetic algorithm as an optimization tool for the development of sponge cell culture media. In Vitro Cell. Dev. Biol. Anim. 55, 149-158 (2019).

104. Van Soest, R. W. et al. Global diversity of sponges (Porifera). PLoS One 7, e35105 (2012).

105. Loh, T. L. \& Pawlik, J. R. Chemical defenses and resource trade-offs structure sponge communities on Caribbean coral reefs. Proc. Natl. Acad. Sci. U. S. A. 111, 4151-4156 (2014).

106. Carstens, B. B. et al. Isolation, Characterization, and Synthesis of the Barrettides: DisulfideContaining Peptides from the Marine Sponge Geodia barretti. J. Nat. Prod. 78, 1886-1893 (2015).

107. Keyzers, R. A. \& Davies-Coleman, M. T. Anti-inflammatory metabolites from marine sponges. Chem. Soc. Rev. 34, 355-365 (2005).

108. Hilge, M., Aelen, J. \& Vuister, G. W. Ca2+ regulation in the Na+/Ca2+ exchanger involves two markedly different Ca2+ sensors. Mol. Cell 22, 15-25 (2006).

109. Martin, V. et al. Differential effects of crambescins and crambescidin 816 in voltage-gated sodium, potassium and calcium channels in neurons. Chem. Res. Toxicol. 26, 169-178 (2013). 
110. Ventura, P. et al. Cnidarian Primary Cell Culture as a Tool to Investigate the Effect of Thermal Stress at Cellular Level. Mar. Biotechnol. (NY) 20, 144-154 (2018).

111. Cai, X. \& Zhang, Y. J. Marine invertebrate cell culture: a decade of development. Oceanography 70 (2014).

112. Laport, M. S., Santos, O. C. \& Muricy, G. Marine sponges: potential sources of new antimicrobial drugs. Curr. Pharm. Biotechnol. 10, 86-105 (2009).

113. Perdicaris, S., Vlachogianni, T. \& Valavanidis, A. Bioactive Natural Substances from Marine Sponges: New Developments and Prospects for Future Pharmaceuticals. Natural Products Chemistry \& Research 1 (2013).

114. de Goeij, J. M., Lesser, M. P. \& Pawlik, J. R. in Climate Change, Ocean Acidification and Sponges (eds Carballo J. \& Bell J.) (Springer, Cham, 2017).

115. De Rosa, S. et al. Development in primary cell culture of demosponges. J. Biotechnol. 100, 119125 (2003).

116. Willoughby, R. \& Pomponi, S. A. Quantitative assessment of marine sponge cells in vitro: development of improved growth medium. In Vitro Cell. Dev. Biol. Anim. 36, 194-200 (2000).

117. de Caralt, S., Uriz, M. J. \& Wijffels, R. H. Cell culture from sponges: pluripotency and immortality. Trends Biotechnol. 25, 467-471 (2007).

118. Pozzolini, M. et al. Influence of rocky substrata on three-dimensional sponge cells model development. In Vitro Cell. Dev. Biol. Anim. 46, 140-147 (2010).

119. Wijffels, R. H., Osinga, R., Pomponi, S. A. \& Tramper, J. in Multiphase Bioreactor Design (eds Cabral, J. M. S., Mota, M. \& Tramper, J.) 477-494 (Taylor \& Francis, London, 2001).

120. Ramsby, B., Massaro, A., Marshall, E., Wilcox, T. \& Hill, M. Epibiont-basibiont interactions: examination of ecological factors that influence specialization in a two-sponge association between Geodia vosmaeri (Sollas, 1886) and Amphimedon erina (de Laubenfels, 1936). Hydrobiologia 687, 331-340 (2012).

121. Naim, M. A., Smidt, H. \& Sipkema, D. Fungi found in Mediterranean and North Sea sponges: how specific are they? PeerJ 5, e3722 (2017).

122. Vainio, E. J. \& Hantula, J. Direct analysis of wood-inhabiting fungi using denaturing gradient gel electrophoresis of amplified ribosomal DNA. Mycological Research 104, 927-936 (2000).

123. Di, X. et al. 6-Bromoindole Derivatives from the Icelandic Marine Sponge Geodia barretti: Isolation and Anti-Inflammatory Activity. Mar. Drugs 16, 10.3390/md16110437 (2018).

124. Freshney, R. I. in (John Wiley \& Sons, Inc., Hoboken, New Jersey, 2016).

125. Schaeffer, W. I. Terminology associated with cell, tissue, and organ culture, molecular biology, and molecular genetics. Tissue Culture Association Terminology Committee. In Vitro Cell. Dev. Biol. 26, 97-101 (1990). 
126. Pita, L., Rix, L., Slaby, B. M., Franke, A. \& Hentschel, U. The sponge holobiont in a changing ocean: from microbes to ecosystems. Microbiome 6, 46-018-0428-1 (2018).

127. Steinert, G., Rohde, S., Janussen, D., Blaurock, C. \& Schupp, P. J. Host-specific assembly of sponge-associated prokaryotes at high taxonomic ranks. Sci. Rep. 7, 2542-017-02656-6 (2017).

128. Araujo, M., Xavier, J. R., Nunes, C. D., Vaz, P. D. \& Humanes, M. Marine sponge melanin: a new source of an old biopolymer. Struct. Chem. 23, 115-122 (2012).

129. Vijayan, V. et al. Sponge-Associated Bacteria Produce Non-cytotoxic Melanin Which Protects Animal Cells from Photo-Toxicity. Appl. Biochem. Biotechnol. 183, 396-411 (2017).

130. Gabbai-Armelin, P. R. et al. Characterization and Cytotoxicity Evaluation of a Marine Sponge Biosilica. Mar. Biotechnol. (NY) 21, 65-75 (2019).

131. Lin, Z. et al. In vitro evaluation of natural marine sponge collagen as a scaffold for bone tissue engineering. Int. J. Biol. Sci. 7, 968-977 (2011).

132. Nandi, S. K. et al. In vitro and in vivo evaluation of the marine sponge skeleton as a bone mimicking biomaterial. Integr. Biol. (Camb) 7, 250-262 (2015).

133. Macha, I. J. \& Ben-Nissan, B. Marine Skeletons: Towards Hard Tissue Repair and Regeneration. Mar. Drugs 16, 10.3390/md16070225 (2018).

134. Granito, R. N., Custodio, M. R. \& Renno, A. C. M. Natural marine sponges for bone tissue engineering: The state of art and future perspectives. J. Biomed. Mater. Res. B. Appl. Biomater. 105, 1717-1727 (2017).

135. Brummer, F. \& Nickel, M. Sustainable use of marine resources: cultivation of sponges. Prog. Mol. Subcell. Biol. 37, 143-162 (2003).

136. Conkling, M. et al. Breakthrough in Marine Invertebrate Cell Culture: Sponge Cells Divide Rapidly in Improved Nutrient Medium. Sci. Rep. 9, 17321-019-53643-y (2019).

137. Komarov, P. G. et al. A chemical inhibitor of p53 that protects mice from the side effects of cancer therapy. Science 285, 1733-1737 (1999).

138. Grabherr, M. G. et al. Full-length transcriptome assembly from RNA-Seq data without a reference genome. Nat. Biotechnol. 29, 644-652 (2011).

139. Langmead, B. \& Salzberg, S. L. Fast gapped-read alignment with Bowtie 2. Nat. Methods 9, 357359 (2012).

140. Hosseini, P., Tremblay, A., Matthews, B. F. \& Alkharouf, N. W. An efficient annotation and geneexpression derivation tool for Illumina Solexa datasets. BMC Res. Notes 3, 183-0500-3-183 (2010).

141. Buchfink, B., Xie, C. \& Huson, D. H. Fast and sensitive protein alignment using DIAMOND. Nat. Methods 12, 59-60 (2015).

142. Moriya, Y., Itoh, M., Okuda, S., Yoshizawa, A. C. \& Kanehisa, M. KAAS: an automatic genome annotation and pathway reconstruction server. Nucleic Acids Res. 35, W182-5 (2007). 
143. Altschul, S. F., Gish, W., Miller, W., Myers, E. W. \& Lipman, D. J. Basic local alignment search tool. J. Mol. Biol. 215, 403-410 (1990).

144. Eddy, S. R. A probabilistic model of local sequence alignment that simplifies statistical significance estimation. PLoS Comput. Biol. 4, e1000069 (2008).

145. Conesa, A. et al. Blast2GO: a universal tool for annotation, visualization and analysis in functional genomics research. Bioinformatics 21, 3674-3676 (2005).

146. Gotz, S. et al. High-throughput functional annotation and data mining with the Blast2GO suite. Nucleic Acids Res. 36, 3420-3435 (2008).

147. Huson, D. H., Auch, A. F., Qi, J. \& Schuster, S. C. MEGAN analysis of metagenomic data. Genome Res. 17, 377-386 (2007).

148. Li, B. \& Dewey, C. N. RSEM: accurate transcript quantification from RNA-Seq data with or without a reference genome. BMC Bioinformatics 12, 323-2105-12-323 (2011).

149. Love, M. I., Huber, W. \& Anders, S. Moderated estimation of fold change and dispersion for RNA-seq data with DESeq2. Genome Biol. 15, 550-014-0550-8 (2014).

150. Young, M. D., Wakefield, M. J., Smyth, G. K. \& Oshlack, A. Gene ontology analysis for RNA-seq: accounting for selection bias. Genome Biol. 11, R14-2010-11-2-r14. Epub 2010 Feb 4 (2010).

151. Alexa, A. \& Rahnenfuhrer, J. Gene set enrichment analysis with topGO. (2007).

152. Mao, X., Cai, T., Olyarchuk, J. G. \& Wei, L. Automated genome annotation and pathway identification using the KEGG Orthology (KO) as a controlled vocabulary. Bioinformatics 21, 37873793 (2005).

153. Xie, C. et al. KOBAS 2.0: a web server for annotation and identification of enriched pathways and diseases. Nucleic Acids Res. 39, W316-22 (2011).

154. Wu, J., Mao, X., Cai, T., Luo, J. \& Wei, L. KOBAS server: a web-based platform for automated annotation and pathway identification. Nucleic Acids Res. 34, W720-4 (2006).

155. Gul, I. S. et al. GC Content of Early Metazoan Genes and Its Impact on Gene Expression Levels in Mammalian Cell Lines. Genome Biol. Evol. 10, 909-917 (2018).

156. Simao, F. A., Waterhouse, R. M., loannidis, P., Kriventseva, E. V. \& Zdobnov, E. M. BUSCO: assessing genome assembly and annotation completeness with single-copy orthologs. Bioinformatics 31, 3210-3212 (2015).

157. Nishimura, O., Hara, Y. \& Kuraku, S. gVolante for standardizing completeness assessment of genome and transcriptome assemblies. Bioinformatics 33, 3635-3637 (2017).

158. Marat, A. L. \& Haucke, V. Phosphatidylinositol 3-phosphates-at the interface between cell signalling and membrane traffic. EMBO J. 35, 561-579 (2016).

159. Kumar, H. \& Choi, D. Hypoxia Inducible Factor Pathway and Physiological Adaptation: A Cell Survival Pathway? Mediators Inflamm. 2015, 584758 (2015). 
160. Tu, C. L. \& You, M. Obligatory roles of filamin A in E-cadherin-mediated cell-cell adhesion in epidermal keratinocytes. J. Dermatol. Sci. 73, 142-151 (2014).

161. Adams, M. et al. A meckelin-filamin A interaction mediates ciliogenesis. Hum. Mol. Genet. 21, 1272-1286 (2012).

162. Cunha, S. R. \& Mohler, P. J. Ankyrin protein networks in membrane formation and stabilization. J. Cell. Mol. Med. 13, 4364-4376 (2009).

163. Barczyk, M., Carracedo, S. \& Gullberg, D. Integrins. Cell Tissue Res. 339, 269-280 (2010).

164. Frantz, C., Stewart, K. M. \& Weaver, V. M. The extracellular matrix at a glance. J. Cell. Sci. 123, 4195-4200 (2010).

165. Hartman, M. A. \& Spudich, J. A. The myosin superfamily at a glance. J. Cell. Sci. 125, 1627-1632 (2012).

166. Parsons, J. T., Horwitz, A. R. \& Schwartz, M. A. Cell adhesion: integrating cytoskeletal dynamics and cellular tension. Nat. Rev. Mol. Cell Biol. 11, 633-643 (2010).

167. Ramos, T. N., Bullard, D. C. \& Barnum, S. R. ICAM-1: isoforms and phenotypes. J. Immunol. 192, 4469-4474 (2014).

168. Mohri, H., Inaba, K., Ishijima, S. \& Baba, S. A. Tubulin-dynein system in flagellar and ciliary movement. Proc. Jpn. Acad. Ser. B. Phys. Biol. Sci. 88, 397-415 (2012).

169. Lodish, H. et al. in Molecular cell biology (Macmillan, 2008).

170. Jouette, J., Guichet, A. \& Claret, S. B. Dynein-mediated transport and membrane trafficking control PAR3 polarised distribution. Elife 8, 10.7554/eLife.40212 (2019).

171. Johansson, M. et al. Activation of endosomal dynein motors by stepwise assembly of Rab7-RILPp150Glued, ORP1L, and the receptor betalll spectrin. J. Cell Biol. 176, 459-471 (2007).

172. Stuchell-Brereton, M. D. et al. Functional interaction between dynein light chain and intermediate chain is required for mitotic spindle positioning. Mol. Biol. Cell 22, 2690-2701 (2011).

173. Gupta, V. et al. GIV/Girdin activates Galphai and inhibits Galphas via the same motif. Proc. Natl. Acad. Sci. U. S. A. 113, E5721-30 (2016).

174. Brunori, M., Antonini, G., Malatesta, F., Sarti, P. \& Wilson, M. T. Cytochrome-c oxidase. Subunit structure and proton pumping. Eur. J. Biochem. 169, 1-8 (1987).

175. Sharma, L. K., Lu, J. \& Bai, Y. Mitochondrial respiratory complex I: structure, function and implication in human diseases. Curr. Med. Chem. 16, 1266-1277 (2009).

176. Wang, Y., Luo, Y., Hong, Y., Peng, J. \& Lo, L. Ribosome biogenesis factor Bms1-like is essential for liver development in zebrafish. J. Genet. Genomics 39, 451-462 (2012).

177. Perry, D. G., Daugherty, G. L. \& Martin, W. J.,2nd. Clathrin-coated pit-associated proteins are required for alveolar macrophage phagocytosis. J. Immunol. 162, 380-386 (1999). 
178. Kirchhausen, T., Owen, D. \& Harrison, S. C. Molecular structure, function, and dynamics of clathrin-mediated membrane traffic. Cold Spring Harb Perspect. Biol. 6, a016725 (2014).

179. Tamura, N. et al. Specific role of phosphoinositide 3-kinase p110alpha in the regulation of phagocytosis and pinocytosis in macrophages. Biochem. J. 423, 99-108 (2009).

180. Zhuang, G., Hunter, S., Hwang, Y. \& Chen, J. Regulation of EphA2 receptor endocytosis by SHIP2 lipid phosphatase via phosphatidylinositol 3-Kinase-dependent Rac1 activation. J. Biol. Chem. 282, 2683-2694 (2007).

181. Szymkiewicz, I. et al. CIN85 participates in Cbl-b-mediated down-regulation of receptor tyrosine kinases. J. Biol. Chem. 277, 39666-39672 (2002).

182. Soubeyran, P., Kowanetz, K., Szymkiewicz, I., Langdon, W. Y. \& Dikic, I. CbI-CIN85-endophilin complex mediates ligand-induced downregulation of EGF receptors. Nature 416, 183-187 (2002).

183. Schmidt, O. \& Teis, D. The ESCRT machinery. Curr. Biol. 22, R116-20 (2012).

184. Rogalska, E., Ransac, S. \& Verger, R. Stereoselectivity of lipases. II. Stereoselective hydrolysis of triglycerides by gastric and pancreatic lipases. J. Biol. Chem. 265, 20271-20276 (1990).

185. Yamasaki, M. et al. Acetoacetyl-CoA synthetase gene is abundant in rat adipose, and related with fatty acid synthesis in mature adipocytes. Biochem. Biophys. Res. Commun. 335, 215-219 (2005).

186. Traut, T. in Allosteric Regulatory Enzymes 127-138 (Springer, Boston, MA, 2008).

187. Gerondopoulos, A., Langemeyer, L., Liang, J. R., Linford, A. \& Barr, F. A. BLOC-3 mutated in Hermansky-Pudlak syndrome is a Rab32/38 guanine nucleotide exchange factor. Curr. Biol. 22, 21352139 (2012).

188. Cooper, G. M. in The Cell: A Molecular Approach (Sinauer Associates, Sunderland (MA), 2000).

189. Cregan, S. P. et al. Bax-dependent caspase-3 activation is a key determinant in p53-induced apoptosis in neurons. J. Neurosci. 19, 7860-7869 (1999).

190. Luo, Y., Hurwitz, J. \& Massague, J. Cell-cycle inhibition by independent CDK and PCNA binding domains in p21Cip1. Nature 375, 159-161 (1995).

191. Rane, C. K. \& Minden, A. P21 activated kinases: structure, regulation, and functions. Small GTPases 5, 10.4161/sgtp.28003. Epub 2014 Mar 21 (2014).

192. Taylor, S. S., Ha, E. \& McKeon, F. The human homologue of Bub3 is required for kinetochore localization of Bub1 and a Mad3/Bub1-related protein kinase. J. Cell Biol. 142, 1-11 (1998).

193. Martinez-Exposito, M. J., Kaplan, K. B., Copeland, J. \& Sorger, P. K. Retention of the BUB3 checkpoint protein on lagging chromosomes. Proc. Natl. Acad. Sci. U. S. A. 96, 8493-8498 (1999).

194. Dornan, D. et al. The ubiquitin ligase COP1 is a critical negative regulator of p53. Nature $429,86-$ 92 (2004). 
195. Li, M., Brooks, C. L., Kon, N. \& Gu, W. A dynamic role of HAUSP in the p53-Mdm2 pathway. Mol. Cell 13, 879-886 (2004).

196. Li, M. et al. Deubiquitination of $\mathrm{p} 53$ by HAUSP is an important pathway for $\mathrm{p} 53$ stabilization. Nature 416, 648-653 (2002).

197. Chwieralski, C. E., Welte, T. \& Buhling, F. Cathepsin-regulated apoptosis. Apoptosis 11, 143-149 (2006).

198. Gondi, C. S. \& Rao, J. S. Cathepsin B as a cancer target. Expert Opin. Ther. Targets 17, 281-291 (2013).

199. Beaujouin, M. \& Liaudet-Coopman, E. Cathepsin D overexpressed by cancer cells can enhance apoptosis-dependent chemo-sensitivity independently of its catalytic activity. Adv. Exp. Med. Biol. 617, 453-461 (2008).

200. Gupta, S. \& Knowlton, A. A. Cytosolic heat shock protein 60, hypoxia, and apoptosis. Circulation 106, 2727-2733 (2002).

201. Ghosh, J. C., Dohi, T., Kang, B. H. \& Altieri, D. C. Hsp60 regulation of tumor cell apoptosis. J. Biol. Chem. 283, 5188-5194 (2008).

202. Jacobs, A. L. \& Schar, P. DNA glycosylases: in DNA repair and beyond. Chromosoma 121, 1-20 (2012).

203. Friedman, J. I. \& Stivers, J. T. Detection of damaged DNA bases by DNA glycosylase enzymes. Biochemistry 49, 4957-4967 (2010).

204. Simsek, D. \& Jasin, M. DNA ligase III: a spotty presence in eukaryotes, but an essential function where tested. Cell. Cycle 10, 3636-3644 (2011).

205. Williams, R. S., Williams, J. S. \& Tainer, J. A. Mre11-Rad50-Nbs1 is a keystone complex connecting DNA repair machinery, double-strand break signaling, and the chromatin template. Biochem. Cell Biol. 85, 509-520 (2007).

206. Ray Chaudhuri, A. \& Nussenzweig, A. The multifaceted roles of PARP1 in DNA repair and chromatin remodelling. Nat. Rev. Mol. Cell Biol. 18, 610-621 (2017).

207. Fouquin, A. et al. PARP2 controls double-strand break repair pathway choice by limiting 53BP1 accumulation at DNA damage sites and promoting end-resection. Nucleic Acids Res. 45, 12325-12339 (2017).

208. Belousova, E. A., Kutuzov, M. M., Ivankina, P. A., Ishchenko, A. A. \& Lavrik, O. I. A New DNA Break Repair Pathway Involving PARP3 and Base Excision Repair Proteins. Dokl. Biochem. Biophys. 482, 233-237 (2018).

209. Wang, Z., Lin, H., Hua, F. \& Hu, Z. W. Repairing DNA damage by XRCC6/KU70 reverses TLR4deficiency-worsened HCC development via restoring senescence and autophagic flux. Autophagy 9 , 925-927 (2013). 
210. Rajavel, M., Mullins, M. R. \& Taylor, D. J. Multiple facets of TPP1 in telomere maintenance. Biochim. Biophys. Acta 1844, 1550-1559 (2014).

211. Venteicher, A. S. et al. A human telomerase holoenzyme protein required for Cajal body localization and telomere synthesis. Science 323, 644-648 (2009).

212. Cheung, D. H., Kung, H. F., Huang, J. J. \& Shaw, P. C. PinX1 is involved in telomerase recruitment and regulates telomerase function by mediating its localization. FEBS Lett. 586, 3166-3171 (2012).

213. Zhou, X. Z. \& Lu, K. P. The Pin2/TRF1-interacting protein PinX1 is a potent telomerase inhibitor. Cell 107, 347-359 (2001).

214. Smith, S., Giriat, I., Schmitt, A. \& de Lange, T. Tankyrase, a poly(ADP-ribose) polymerase at human telomeres. Science 282, 1484-1487 (1998).

215. Robinson, G. W. \& Hennighausen, L. Inhibins and activins regulate mammary epithelial cell differentiation through mesenchymal-epithelial interactions. Development 124, 2701-2708 (1997).

216. DePaolo, L. V., Bicsak, T. A., Erickson, G. F., Shimasaki, S. \& Ling, N. Follistatin and activin: a potential intrinsic regulatory system within diverse tissues. Proc. Soc. Exp. Biol. Med. 198, 500-512 (1991).

217. Davidson, K. C. et al. Wnt/beta-catenin signaling promotes differentiation, not self-renewal, of human embryonic stem cells and is repressed by Oct4. Proc. Natl. Acad. Sci. U. S. A. 109, 4485-4490 (2012).

218. Lonic, A. et al. Fibroblast growth factor receptor 2 phosphorylation on serine 779 couples to 14 3-3 and regulates cell survival and proliferation. Mol. Cell. Biol. 28, 3372-3385 (2008).

219. Szybowska, P., Kostas, M., Wesche, J., Wiedlocha, A. \& Haugsten, E. M. Cancer Mutations in FGFR2 Prevent a Negative Feedback Loop Mediated by the ERK1/2 Pathway. Cells 8, 10.3390/cells8060518 (2019).

220. Conaco, C. et al. Detection of Prokaryotic Genes in the Amphimedon queenslandica Genome. PLoS One 11, e0151092 (2016).

221. Taylor, M. W., Radax, R., Steger, D. \& Wagner, M. Sponge-associated microorganisms: evolution, ecology, and biotechnological potential. Microbiol. Mol. Biol. Rev. 71, 295-347 (2007).

222. Simister, R. L., Deines, P., Botte, E. S., Webster, N. S. \& Taylor, M. W. Sponge-specific clusters revisited: a comprehensive phylogeny of sponge-associated microorganisms. Environ. Microbiol. 14, 517-524 (2012).

223. Handy, D. E. \& Loscalzo, J. Redox regulation of mitochondrial function. Antioxid. Redox Signal. 16, 1323-1367 (2012).

224. Heng, Y. W. \& Koh, C. G. Actin cytoskeleton dynamics and the cell division cycle. Int. J. Biochem. Cell Biol. 42, 1622-1633 (2010).

225. Livne, A. \& Geiger, B. The inner workings of stress fibers - from contractile machinery to focal adhesions and back. J. Cell. Sci. 129, 1293-1304 (2016). 
226. Mattila, P. K. \& Lappalainen, P. Filopodia: molecular architecture and cellular functions. Nat. Rev. Mol. Cell Biol. 9, 446-454 (2008).

227. Roberts, A. J., Kon, T., Knight, P. J., Sutoh, K. \& Burgess, S. A. Functions and mechanics of dynein motor proteins. Nat. Rev. Mol. Cell Biol. 14, 713-726 (2013).

228. Gibbons, B. H., Asai, D. J., Tang, W. J., Hays, T. S. \& Gibbons, I. R. Phylogeny and expression of axonemal and cytoplasmic dynein genes in sea urchins. Mol. Biol. Cell 5, 57-70 (1994).

229. Varner, J. A. Isolation of a sponge-derived extracellular matrix adhesion protein. J. Biol. Chem. 271, 16119-16125 (1996).

230. Ozbek, S., Balasubramanian, P. G., Chiquet-Ehrismann, R., Tucker, R. P. \& Adams, J. C. The evolution of extracellular matrix. Mol. Biol. Cell 21, 4300-4305 (2010).

231. Grice, L. F. et al. Origin and Evolution of the Sponge Aggregation Factor Gene Family. Mol. Biol. Evol. 34, 1083-1099 (2017).

232. Blumbach, B. et al. The putative sponge aggregation receptor. Isolation and characterization of a molecule composed of scavenger receptor cysteine-rich domains and short consensus repeats. J. Cell. Sci. 111 ( Pt 17), 2635-2644 (1998).

233. Wilson, H. V. On some phenomena of coalescence and regeneration in sponges. Journal of Experimental Zoology 5 (1907).

234. Muller, W. E., Zahn, R. K., Kurelec, B. \& Muller, I. Species-specific aggregation factor in sponges : VIII. Nature and alteration of cell surface charge. Wilehm Roux. Arch. Dev. Biol. 184, 29-40 (1978).

235. Muller, H. et al. Deleted in malignant brain tumors 1 is present in the vascular extracellular matrix and promotes angiogenesis. Arterioscler. Thromb. Vasc. Biol. 32, 442-448 (2012).

236. Wang, Z., Dillon, J. \& Gaillard, E. R. Antioxidant properties of melanin in retinal pigment epithelial cells. Photochem. Photobiol. 82, 474-479 (2006).

237. Page, S., Chandhoke, V. \& Baranova, A. Melanin and melanogenesis in adipose tissue: possible mechanisms for abating oxidative stress and inflammation? Obes. Rev. 12, e21-31 (2011).

238. Kumar, C. G., Mongolla, P., Pombala, S., Kamle, A. \& Joseph, J. Physicochemical characterization and antioxidant activity of melanin from a novel strain of Aspergillus bridgeri ICTF-201. Lett. Appl. Microbiol. 53, 350-358 (2011).

239. Nosanchuk, J. D. \& Casadevall, A. Impact of melanin on microbial virulence and clinical resistance to antimicrobial compounds. Antimicrob. Agents Chemother. 50, 3519-3528 (2006).

240. Nosanchuk, J. D. \& Casadevall, A. The contribution of melanin to microbial pathogenesis. Cell. Microbiol. 5, 203-223 (2003).

241. McDonald, A. E. Alternative oxidase: what information can protein sequence comparisons give us? Physiol. Plant. 137, 328-341 (2009). 
242. Harrington, L. et al. Human telomerase contains evolutionarily conserved catalytic and structural subunits. Genes Dev. 11, 3109-3115 (1997).

243. Harrington, L. et al. A mammalian telomerase-associated protein. Science 275, 973-977 (1997).

244. Cong, Y. S., Wright, W. E. \& Shay, J. W. Human telomerase and its regulation. Microbiol. Mol. Biol. Rev. 66, 407-25, table of contents (2002).

245. Shay, J. W. \& Bacchetti, S. A survey of telomerase activity in human cancer. Eur. J. Cancer 33, 787-791 (1997).

246. Cetkovic, H., Halasz, M. \& Herak Bosnar, M. Sponges: A Reservoir of Genes Implicated in Human Cancer. Mar. Drugs 16, 10.3390/md16010020 (2018).

247. Jochum, K. P., Wang, X., Vennemann, T. W., Sinha, B. \& Müller, W. E. G. Siliceous deep-sea sponge Monorhaphis chuni: A potential paleoclimate archive in ancient animals. Chemical Geology 300-301, 143-151 (2012).

248. Petralia, R. S., Mattson, M. P. \& Yao, P. J. Aging and longevity in the simplest animals and the quest for immortality. Ageing Res. Rev. 16, 66-82 (2014).

249. Fogh, J. :. T., G. in Human Tumor Cells in Vitro. (ed Fogh, J.) (Springer, Boston, MA., 1975).

250. Zahn, R. K. et al. DNA damage by PAH and repair in a marine sponge. Sci. Total Environ. 26, 137156 (1983).

251. Schwartsmann, G., Da Rocha, A. B., Mattei, J. \& Lopes, R. Marine-derived anticancer agents in clinical trials. Expert Opin. Investig. Drugs 12, 1367-1383 (2003).

252. Nuijen, B. et al. Pharmaceutical development of anticancer agents derived from marine sources. Anticancer Drugs 11, 793-811 (2000).

253. Alcaraz, M. J. \& Paya, M. Marine sponge metabolites for the control of inflammatory diseases. Curr. Opin. Investig Drugs 7, 974-979 (2006).

254. Qi, S. H. \& Ma, X. Antifouling Compounds from Marine Invertebrates. Mar. Drugs 15, 10.3390/md15090263 (2017).

255. Balboa, E. M., Conde, E., Soto, M. L., Perez-Armada, L. \& Dominguez, H. in Springer handbook of marine biotechnology (ed Kim, S. (. ).) 1015-1042 (Springer, Berlin, 2015).

256. Wang, B., Pfeiffer, M. J., Schwarzer, C., Arauzo-Bravo, M. J. \& Boiani, M. DNA replication is an integral part of the mouse oocyte's reprogramming machinery. PLoS One 9, e97199 (2014).

257. Horvath, P. \& Barrangou, R. CRISPR/Cas, the immune system of bacteria and archaea. Science 327, $167-170$ (2010).

258. Liu, M. et al. Methodologies for Improving HDR Efficiency. Front. Genet. 9, 691 (2019).

259. Jinek, M. et al. A programmable dual-RNA-guided DNA endonuclease in adaptive bacterial immunity. Science 337, 816-821 (2012). 
260. Zetsche, B. et al. Cpf1 is a single RNA-guided endonuclease of a class 2 CRISPR-Cas system. Cell 163, 759-771 (2015).

261. Swarts, D. C. \& Jinek, M. Cas9 versus Cas12a/Cpf1: Structure-function comparisons and implications for genome editing. Wiley Interdiscip. Rev. RNA, e1481 (2018).

262. Safari, F., Zare, K., Negahdaripour, M., Barekati-Mowahed, M. \& Ghasemi, Y. CRISPR Cpf1 proteins: structure, function and implications for genome editing. Cell. Biosci. 9, 36-019-0298-7. eCollection 2019 (2019).

263. Vallmann, K. et al. Expressed 2-5A synthetase genes and pseudogenes in the marine sponge Geodia barretti. Gene 478, 42-49 (2011).

264. Hur, J. K. et al. Targeted mutagenesis in mice by electroporation of Cpf1 ribonucleoproteins. Nat. Biotechnol. 34, 807-808 (2016).

265. Liu, P. et al. Enhanced Cas12a editing in mammalian cells and zebrafish. Nucleic Acids Res. 47, 4169-4180 (2019).

266. Kim, H. et al. CRISPR/Cpf1-mediated DNA-free plant genome editing. Nat. Commun. 8, 14406 (2017).

267. Yoshimi, K. et al. ssODN-mediated knock-in with CRISPR-Cas for large genomic regions in zygotes. Nat. Commun. 7, 10431 (2016).

268. Ferenczi, A., Pyott, D. E., Xipnitou, A. \& Molnar, A. Efficient targeted DNA editing and replacement in Chlamydomonas reinhardtii using Cpf1 ribonucleoproteins and single-stranded DNA. Proc. Natl. Acad. Sci. U. S. A. 114, 13567-13572 (2017).

269. Okamoto, S., Amaishi, Y., Maki, I., Enoki, T. \& Mineno, J. Highly efficient genome editing for single-base substitutions using optimized ssODNs with Cas9-RNPs. Sci. Rep. 9, 4811-019-41121-4 (2019).

270. Revilla-I-Domingo, R., Schmidt, C., Zifko, C. \& Raible, F. Establishment of Transgenesis in the Demosponge Suberites domuncula. Genetics 210, 435-443 (2018).

271. Bier, E., Harrison, M. M., O'Connor-Giles, K. M. \& Wildonger, J. Advances in Engineering the Fly Genome with the CRISPR-Cas System. Genetics 208, 1-18 (2018).

272. Zhang, J. P. et al. Efficient precise knockin with a double cut HDR donor after CRISPR/Cas9mediated double-stranded DNA cleavage. Genome Biol. 18, 35-017-1164-8 (2017).

273. Li, H. et al. Applications of genome editing technology in the targeted therapy of human diseases: mechanisms, advances and prospects. Signal. Transduct Target Ther. 5, 1-019-0089-y. eCollection 2020 (2020).

274. Zhang, Y., Pribil, M., Palmgren, M. \& Gao, C. A CRISPR way for accelerating improvement of food crops. Nature Food 1, 200-205 (2020).

275. Zhang, C., Quan, R. \& Wang, J. Development and application of CRISPR/Cas9 technologies in genomic editing. Hum. Mol. Genet. 27, R79-R88 (2018). 
276. Moon, S. B., Kim, D. Y., Ko, J. H. \& Kim, Y. S. Recent advances in the CRISPR genome editing tool set. Exp. Mol. Med. 51, 1-11 (2019).

277. Sadler, A. J. \& Williams, B. R. Interferon-inducible antiviral effectors. Nat. Rev. Immunol. 8, 559568 (2008).

278. Kristiansen, H., Gad, H. H., Eskildsen-Larsen, S., Despres, P. \& Hartmann, R. The oligoadenylate synthetase family: an ancient protein family with multiple antiviral activities. J. Interferon Cytokine Res. 31, 41-47 (2011).

279. Mullan, P. B. et al. The 2,5 oligoadenylate synthetase/RNaseL pathway is a novel effector of BRCA1- and interferon-gamma-mediated apoptosis. Oncogene 24, 5492-5501 (2005).

280. Kuang, Y., Liu, S., Wang, C. \& Hu, X. Low expression of 2'-5' oligoadenylate synthetase 1 predicted poor prognosis in colorectal cancer. 10, 3353-3360 (2017).

281. Mandal, S. K. \& Chaudhary, J. The expression of $2^{\prime}-5^{\prime}$ oligoadenylate synthetase in prostate cancer and its effect on prostate cancer cell cycle. Cancer Res. 72 (2012).

282. Song, F. \& Stieger, K. Optimizing the DNA Donor Template for Homology-Directed Repair of Double-Strand Breaks. Mol. Ther. Nucleic Acids 7, 53-60 (2017).

283. Shin, J., Chen, J. \& Solnica-Krezel, L. Efficient homologous recombination-mediated genome engineering in zebrafish using TALE nucleases. Development 141, 3807-3818 (2014).

284. Kanca, O. et al. An efficient CRISPR-based strategy to insert small and large fragments of DNA using short homology arms. Elife 8, 10.7554/eLife.51539 (2019).

285. Zhang, J. P. et al. Efficient precise knockin with a double cut HDR donor after CRISPR/Cas9mediated double-stranded DNA cleavage. Genome Biol. 18, 35-017-1164-8 (2017).

286. Santerre, R. F., Allen, N. E., Hobbs, J. N.Jr, Rao, R. N. \& Schmidt, R. J. Expression of prokaryotic genes for hygromycin B and G418 resistance as dominant-selection markers in mouse L cells. Gene 30, 147-156 (1984).

287. Rinkevich, B. Cell cultures from marine invertebrates: obstacles, new approaches and recent improvements. J. Biotechnol. 70, 133-153 (1999).

288. Conkling, M. \&Pomponi, S. A. One sponge, two sponge, red sponge, blue sponge: identifying variation of cell division in cultures of marine demosponges (Proceedings, Society for In Vitro Biology Annual Meeting, 2020).

289. Alexander, B. E. et al. Cell turnover and detritus production in marine sponges from tropical and temperate benthic ecosystems. PLoS One 9, e109486 (2014).

290. De Goeij, J. M. et al. Cell kinetics of the marine sponge Halisarca caerulea reveal rapid cell turnover and shedding. J. Exp. Biol. 212, 3892-3900 (2009).

291. Jeffery, N. W., Jardine, C. B. \& Gregory, T. R. A first exploration of genome size diversity in sponges. Genome 56, 451-456 (2013). 
292. Piovesan, A. et al. On the length, weight and GC content of the human genome. BMC Res. Notes 12, 106-019-4137-z (2019).

293. Groeneveld, P., Stouthamer, A. H. \& Westerhoff, H. V. Super life - how and why 'cell selection' leads to the fastest-growing eukaryote. The FEBS Journal 276, 254-270 (2009).

294. Maldonado, M. Choanoflagellates, choanocytes, and animal multicellularity. Invertebr. Biol. 123, $1-22$ (2004).

295. Nielsen, C. Six major steps in animal evolution: are we derived sponge larvae? Evol. Dev. 10, 241-257 (2008).

296. Carr, M., Leadbeater, B. S. \& Baldauf, S. L. Conserved meiotic genes point to sex in the choanoflagellates. J. Eukaryot. Microbiol. 57, 56-62 (2010).

297. Steenkamp, E. T., Wright, J. \& Baldauf, S. L. The protistan origins of animals and fungi. Mol. Biol. Evol. 23, 93-106 (2006).

298. Naumann, B. \& Burkhardt, P. Spatial Cell Disparity in the Colonial Choanoflagellate Salpingoeca rosetta. Front. Cell. Dev. Biol. 7, 231 (2019).

299. Leadbeater, B. S. C. Life-history and ultrastructure of a new marine species of Proterospongia (Choanoflagellida). J. Mar. Biol. Assoc. U. K. 63, 135-160 (1983).

300. Levin, T. C. \& King, N. Evidence for sex and recombination in the choanoflagellate Salpingoeca rosetta. Curr. Biol. 23, 2176-2180 (2013).

301. David, C. N. \& Campbell, R. D. Cell cycle kinetics and development of Hydra attenuata. I. Epithelial cells. J. Cell. Sci. 11, 557-568 (1972).

302. CURTIS, S. K. \& COWDEN, R. R. Some Aspects of Regeneration in the Scyphistoma of Cassiopea (Class Scyphozoa) as Revealed by the Use of Antimetabolites and Microspectrophotometry. Am. Zool. 14, 851-866 (2015).

303. Alexander, B. E. et al. Cell kinetics during regeneration in the sponge Halisarca caerulea: how local is the response to tissue damage? PeerJ 3, e820 (2015).

304. Ayling, A. L. Growth and Regeneration Rates in Thinly Encrusting Demospongiae from Temperate Waters. Biol. Bull. 165, 343-352 (1983).

305. Harrison, F. W. \& Cowden, R. R. Feulgen microspectrophotometric analysis of deoxyribonucleoprotein organization in larval and adult freshwater sponge nuclei. J. Exp. Zool. 193, 131-135 (1975).

306. Leibfried-Rutledge, M. L., Critser, E. S. \& First, N. L. Effects of fetal calf serum and bovine serum albumin on in vitro maturation and fertilization of bovine and hamster cumulus-oocyte complexes. Biol. Reprod. 35, 850-857 (1986).

307. Jafri, M. A., Ansari, S. A., Alqahtani, M. H. \& Shay, J. W. Roles of telomeres and telomerase in cancer, and advances in telomerase-targeted therapies. Genome Med. 8, 69-016-0324-x (2016). 
308. de Goeij, J. M., Moodley, L., Houtekamer, M., Carballeira, N. M. \& van Duyl, F. C. Tracing 13Cenriched dissolved and particulate organic carbon in the bacteria-containing coral reef sponge Halisarca caerulea: Evidence for DOM-feeding. Limnol. Oceanogr. 53, 1376-1386 (2008).

309. Marieke, K. \& Wijffels, R. Seasonal Growth Rate of the Sponge Haliclona oculata (Demospongiae: Haplosclerida). Marine biotechnology (New York, N. Y. ) 10, 502-10 (2008).

310. Sogabe, S. et al. Pluripotency and the origin of animal multicellularity. Nature 570, 519-522 (2019).

311. Funayama, N., Nakatsukasa, M., Hayashi, T. \& Agata, K. Isolation of the choanocyte in the fresh water sponge, Ephydatia fluviatilis and its lineage marker, Ef annexin. Dev. Growth Differ. 47, 243253 (2005).

312. Funayama, N., Nakatsukasa, M., Mohri, K., Masuda, Y. \& Agata, K. Piwi expression in archeocytes and choanocytes in demosponges: insights into the stem cell system in demosponges. Evol. Dev. 12, 275-287 (2010).

313. Funayama, N. et al. Isolation of Ef silicatein and Ef lectin as molecular markers for sclerocytes and cells involved in innate immunity in the freshwater sponge Ephydatia fluviatilis. Zoolog Sci. 22, 1113-1122 (2005).

314. Horii, T. \& Hatada, I. Challenges to increasing targeting efficiency in genome engineering. J. Reprod. Dev. 62, 7-9 (2016).

315. Yang, H. et al. One-step generation of mice carrying reporter and conditional alleles by CRISPR/Cas-mediated genome engineering. Cell 154, 1370-1379 (2013).

316. Ma, Y. et al. Generating rats with conditional alleles using CRISPR/Cas9. Cell Res. 24, 122-125 (2014).

317. Quadros, R. M. et al. Easi-CRISPR: a robust method for one-step generation of mice carrying conditional and insertion alleles using long ssDNA donors and CRISPR ribonucleoproteins. Genome Biol. 18, 92 (2017).

318. Miura, H., Gurumurthy, C. B., Sato, T., Sato, M. \& Ohtsuka, M. CRISPR/Cas9-based generation of knockdown mice by intronic insertion of artificial microRNA using longer single-stranded DNA. Sci. Rep. 5, 12799 (2015).

319. Cavalier-Smith, T. Origin of animal multicellularity: precursors, causes, consequences-the choanoflagellate/sponge transition, neurogenesis and the Cambrian explosion. Philos. Trans. R. Soc. Lond. B. Biol. Sci. 372, 10.1098/rstb.2015.0476 (2017).

320. Littlewood, D. T. J. Animal Evolution: Last Word on Sponges-First? Curr. Biol. 27, R259-R261 (2017).

321. Schippers, K. J. Sponge Cell Culture. (2013).

322. Rivera, A. S. et al. RNA interference in marine and freshwater sponges: actin knockdown in Tethya wilhelma and Ephydatia muelleri by ingested dsRNA expressing bacteria. BMC Biotechnol. 11, 67-6750-11-67 (2011). 
323. Pirofski, L., Casadevall, A., Rodriguez, L., Zuckier, L. S. \& Scharff, M. D. Current state of the hybridoma technology. J. Clin. Immunol. 10, 5S-12S; discussion 12S-14S (1990).

324. Sieblist, C., Jenzsch, M. \& Pohlscheidt, M. Equipment characterization to mitigate risks during transfers of cell culture manufacturing processes. Cytotechnology 68, 1381-1401 (2016).

325. Murhammer, D. W. \& Goochee, C. F. Sparged animal cell bioreactors: mechanism of cell damage and Pluronic F-68 protection. Biotechnol. Prog. 6, 391-397 (1990).

326. van der Pol, L. A., Paijens, I. \& Tramper, J. Dextran as protectant against damage caused by sparging for hybridoma cells in a bubble column. J. Biotechnol. 43, 103-110 (1995).

327. Yeshanew, M. M. et al. Start-up of an anaerobic fluidized bed reactor treating synthetic carbohydrate rich wastewater. J. Environ. Manage. 184, 456-464 (2016).

328. Perez, M., Rodriguez-Cano, R., Romero, L. I. \& Sales, D. Performance of anaerobic thermophilic fluidized bed in the treatment of cutting-oil wastewater. Bioresour. Technol. 98, 3456-3463 (2007).

329. Tripathi, N. K. \& Shrivastava, A. Recent Developments in Bioprocessing of Recombinant Proteins: Expression Hosts and Process Development. Front. Bioeng. Biotechnol. 7, 420 (2019).

330. Yang, J. et al. Large-scale microcarrier culture of HEK293T cells and Vero cells in single-use bioreactors. AMB Express 9, 70-019-0794-5 (2019).

331. Zhang, L. et al. Microcarrier bioreactor culture system promotes propagation of human intervertebral disc cells. Ir. J. Med. Sci. 179, 529-534 (2010).

332. Wung, N., Acott, S. M., Tosh, D. \& Ellis, M. J. Hollow fibre membrane bioreactors for tissue engineering applications. Biotechnol. Lett. 36, 2357-2366 (2014).

333. Proksch, P., Edrada, R. A. \& Ebel, R. Drugs from the seas - current status and microbiological implications. Appl. Microbiol. Biotechnol. 59, 125-134 (2002). 


\section{Acknowledgements}

First of all I would like to thank Shirley. Of course you are my promotor, but that doesn't even begin to cover it. Over the years you've become not only my mentor, but also a dear friend. When I followed your class on marine biotechnology during my Master in Wageningen, you already started pulling me into your vortex of enthusiasm and passion for sponge research. You gave me the opportunity to do my thesis research with you at Harbor Branch in Florida, and of course I jumped on it. It was a great experience, and I truly appreciate the amount of time you spent teaching me and working with me despite your crazy schedule. While we were on a sampling trip in the beautiful Florida Keys, we started talking about a possible follow-up in the form of a PhD project. I'm very happy that plan came to fruition. You continue to inspire me as a scientist and as a person. Without you I wouldn't have been able to do any of it and I'm incredibly grateful. We hit some bumps along the way, but I'm thankful we came out stronger the other end. I look forward to many more years of friendship.

Rene, thank you for being my promotor and for allowing me to perform my PhD research at the Bioprocess Engineering group. I've felt at home here, largely because of the informal and friendly atmosphere that you created together with the rest of the staff. I've always valued our progress meetings and your perspective on the various aspects of my project. I also want to give a big thanks to my co-promotor, Dirk. I really benefitted from our spontaneous progress meetings (i.e. me walking into your office to ask something, and then ending up discussing my project for half an hour). You always provided a source of calm and a grounding effect, and helped me put things in perspective when necessary.

I also want to thank my paranymphs. Alice, my sestra, I'm so happy you decided to come back to do your PhD research at WUR after your Master, and I hope you'll stick around until forever (unicorns and rainbows everywhere!). Christian, we had many fun times together in and outside of BPE. You were also always there to support me when I needed someone to talk to. You're a great friend. You, Annika, myself and Jesse should still go out for that double date sometime. 
That brings me to thanking all the colleagues at BPE, many of whom have become friends. Thanks to my first office mates Youri, Aziz and Agi, and later on Calvin, Jort and Jin for all the good times in- and outside of the office. On many occasions a group of us at BPE would go for drinks and snacks, whether at the Spot, the Bongerd, in the city centre or at someone's place, with Christian pouring drinks for everyone whether they wanted it or not, Fabian's dangerous cocktails, Enrico belly-dancing and more. Iulian and Judit, we haven't known each other very long, but I feel like we have. I'm glad to call you both friends, and I'm so happy Judit decided to join our crazy Jellatech journey! Anna \& Pauline, over the years we've had some great girls' nights with cocktails, laughs and serious girl talk. Narcis, thank you for showing us the way of the Calçotada, and for being the fearless capitan of FC Bravo. Catalina, I had a great time visiting you in beautiful Aveiro with Pauline, Camilo and Pieter, and that time we randomly ran into each other in Bergen, and we ended up going out for dinner after you and Jeroen gave me a full guided tour of the city. Alex, thank you for showing us around in your amazing mountain- and forest-covered country Slovenia, where we climbed many stairs :) and had many drinks and great food. August \& Chungzhe, thank you for teaching us how to become dumpling masters, and Camilo \& Pauline for being our gracious hosts that evening.

We had many other fun trips, evenings and outings with the BPE crew, like the 2018 PhD trip to San Diego I organised together with my committee mates Edgar, Catalina and Enrico, with guidance from Maria and Sarah. The labuitjes, one of which I organised together with Anna, Pieter and Christian (best labuitje ever!), multiple weekends of Christmas market in Osnabrück and a shared birthday party weekend with Anna and Christian at Anna's parents' place in Germany.

Then there are my students (minions :)), whose efforts significantly contributed to my research and to my growth as a supervisor, scientist and person. Matteo, Lukas, John, Jip, Jans, Ana-Maria and Mitchel: thank you for all your hard work, input and initiative. It was a pleasure to see you grow over the course of your projects, and l've learned a lot from each of you. 
To the rest of the BPE family, thank you for all the good times at coffee breaks, lunches at Orion, Christmas dinners, BPE borrels, for your input at presentations and group meetings, and for being willing to help anyone who asks.

I also received a lot of help from Tom Schonewille at SSB with my RNA isolations using the Maxwell robot that made my life so much easier. And of course I should not forget Mihris, who provided the Cpf1 enzyme for my molecular work.

Next, I would like to thank all the members of the H2020 SponGES consortium. Our yearly General Assembly meetings were not only inspirational and educational, but also very fun, in large part thanks to Joana for orchestrating everything. I am so grateful to have known Hans Tore. You led the consortium in such an informal and friendly manner, and personally took the time to help me collect new samples and transport another batch of samples yourself. Your relentless optimism still inspires me. Rest in peace, you are dearly missed.

Erik thank you for donating and transporting my first $G$. barretti sponge from Bergen. Ana and Vassia, you were always willing to help and collaborate, provided me with $G$. barretti transcriptome data and helped me with my own transcriptome analyses. Detmer, Menia and Catarina, thank you for your advice on and help with RNA isolations from my samples.

Francisca, Vassia, Karin, Menia and Kenny, thank you for lots of fun during General Assembly meetings and in off-time afterwards. Vassia, I'm sorry you fell off my horse that time, even though he was being really good ;). Francisca, thanks for the good times when I visited Bergen to collect samples.

I also spent quite some time at $\mathrm{HBOI}$ in Fort Pierce, Florida, and met some great people there, many of whom helped me at any chance they could. Megan, thank you for your meticulous organization of the sponge biotech lab, and for showing me the ropes in the lab and in the field during my first MSc thesis. Thanks to Stephanie for supervising me at BPE during my second MSc thesis at BPE, for allowing me to try your medium on my cells, and of course for the fun times in Wageningen and Florida. A big thanks to Nick Dickens for taking multiple afternoons out of his busy schedule to give me a crash course in bioinformatics. 
Natuurlijk is er ook mijn familie. Mam en pap, bedankt voor jullie onvoorwaardelijke liefde en steun. Ayla, ik ben blij dat we zo'n sterke band hebben ontwikkeld. Bedankt dat je de beste grote zus bent, en een vriendin om alles mee te delen. Casper en James, mijn grote kleine bro's, en Myriam, mijn mini-me, ik zal altijd jullie trotse grote zus zijn. Opa Ger, mijn lieve grootvader die altijd voor me klaar staat en Oma Ria, die dat ook deed toen ze nog bij ons was. Oma Ina, ik zie u niet vaak maar ik ben dankbaar dat u er bent. Ma en Pa, jullie zijn de beste schoonouders die ik mij kan wensen, en Pa bedankt dat je mij een schop onder mijn kont gaf toen ik het nodig had. Dana en Geralt, liefste schoonzus en zwager, en Eliza, Nadieh, Tygo \& Aalt de liefste nichtjes en neefjes. Besides my family by blood and family by marriage, I'm blessed enough to have a third; a family by choice. Cathy and Joe, Gram and Jay, Adam, Kat, and Ethan, J4, and the rest of the Rossmell/Good family, thank you so much for taking me in and accepting me as one of your own. You are the most kind-hearted people I've ever met, and I'm so very grateful to know you. I don't get to spend nearly as much time with you as I'd like, due to the distance between us. Skype helps, but we'll have to keep visiting each other, because real life is just much better!

Then there are a lot of friends outside of work I would like to thank for fun times and often also for being there when times weren't so fun. My study friends from the MIB thesis time and afterwards, Nicolò, Marta, Boris, Enrique and Yahya, Foskea and Fer. We should have dinner together soon!

Ruben, we've been good friends for nearly 15 years now. You've always been there when I needed you, and I hope we'll get to spend many more evenings playing D\&D, drinking beer and watching stupid videos together with Jesse, Ferdie, April and (hopefully) Jorrit.

Last but not least, there's Jesse. You have been my rock ever since we found each other, and your undying love and support have gotten me through the darkest periods of my life. It's safe to say that I wouldn't have been able to get where I am today without you. I love you more than words can say, and I can't wait to spend the rest of our lives together. Thank you, from the bottom of my heart, for everything. 


\section{Curriculum Vitae}

Kylie Hesp was born on May $9^{\text {th }} 1991$ in Gouda, the Netherlands. At 5 years old, she moved to Biddinghuizen (Flevoland) with her parents, where she later attended primary school 'de Wingerd'. She started high school in the Dronten location of ' $t$ Ichthus college, and graduated cum laude from her gymnasium (Alevels) in $\mathbf{2 0 0 9}$ at the Kampen location of the same school. She started her higher education with a Bachelor in Veterinary Medicine, but discovered after some time that her true passion lay elsewhere: in biotechnology. Therefore the MSc Biotechnology in Wageningen was the perfect place for her, and she immediately felt at home in the city and at the university. During her Master she worked on 3 MSc theses, two of which were in sponge biotechnology: one at the Pomponi lab of Harbor Branch Oceanographic Institute in Ft.

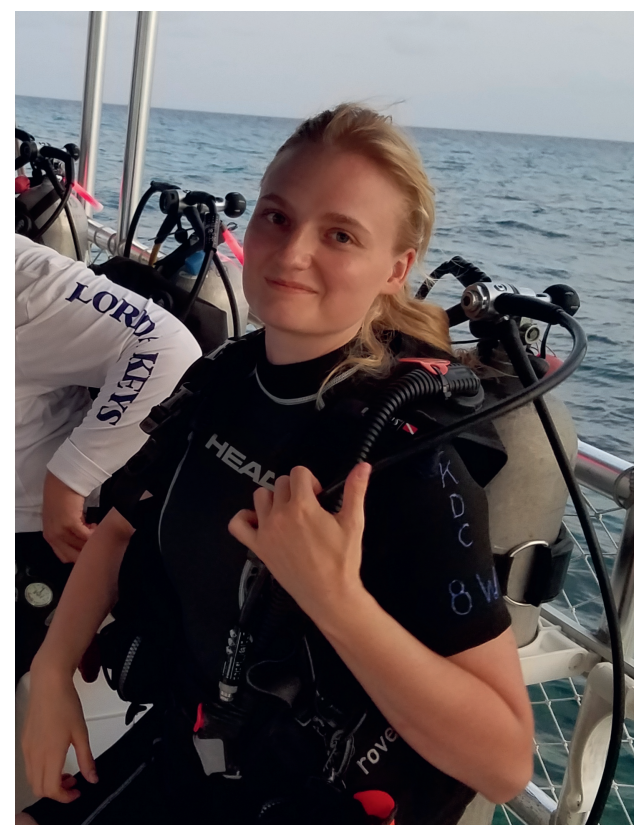

Pierce, Florida, followed by a project at the Bioprocess Engineering group at Wageningen University. In the third thesis she expanded her knowledge on molecular techniques such as gene-editing systems, CRISPR-Cas specifically, by spending time with Stan Brouns' group at the Microbiology department in Wageningen. She finished her MSc in 2016 with a double specialization, Marine Biotechnology and Medical Biotechnology, both with distinction. She then immediately started her PhD research titled 'Dawn of a New Era in Sponge Biotechnology'. 


\section{Overview of completed training activities}

\section{Discipline specific activities}

Open Water Diver course (2016)

Rewriting our genes (2016)

$10^{\text {th }}$ World Sponge Conference ${ }^{1}$, Galway (2017)

Advanced Adventurer Dive course (2017)

SponGES General Assembly meetings

- London $^{1}$ (2017)

- Porto $^{1}(2018)$

- Wageningen ${ }^{1}$ (2019)

- Azoren $^{1}(2020)$

\section{General courses}

PhD week VLAG (2016)

Getting on track with your Veni application (2019)

Career assessment (2019/2020)

Scientific Artwork, Data visualisation and Infographics with Adobe Illustrator (2020)

Brain Training (2020)

Scientific Writing (2020)

\section{Optionals}

Preparation of research proposal

PhD trip San Diego ${ }^{3}$ (2018)

BPE group meetings (2016-2020)

\footnotetext{
${ }^{1}$ Presentation

${ }^{2}$ Poster

${ }^{3}$ Organization
} 
The study was carried out at the department of Bioprocess Engineering of Wageningen University and Research, Wageningen, the Netherlands. The research described in this thesis was financially supported by the European Union Horizon 2020 Project SponGES (grant agreement No. 679848).

This thesis was printed by Digiforce ProefschriftMaken.nl on $100 \%$ recycled paper.

Cover art by Sanne van der Velde. 

